\title{
Ultrafast dynamics during the photoinduced phase transition in $\mathrm{VO}_{2}$
}

\author{
Daniel Wegkamp, Julia Stähler* \\ Department of Physical Chemistry, Fritz-Haber-Institute of the Max Planck Society, Faradayweg 4-6, 14195 Berlin, Germany
}

\begin{abstract}
The phase transition of $\mathrm{VO}_{2}$ from a monoclinic insulator to a rutile metal, which occurs thermally at $T_{\mathrm{C}}=340 \mathrm{~K}$, can also be driven by strong photoexcitation. The ultrafast dynamics during this photoinduced phase transition (PIPT) have attracted great scientific attention for decades, as this approach promises to answer the question of whether the insulator-to-metal (IMT) transition is caused by electronic or crystallographic processes through disentanglement of the different contributions in the time domain. We review our recent results achieved by femtosecond time-resolved photoelectron, optical, and coherent phonon spectroscopy and discuss them within the framework of a selection of latest, complementary studies of the ultrafast PIPT in $\mathrm{VO}_{2}$. We show that the population change of electrons and holes caused by photoexcitation launches a highly non-equilibrium plasma phase characterized by enhanced screening due to quasi-free carriers and followed by two branches of non-equilibrium dynamics: (i) an instantaneous (within the time resolution) collapse of the insulating gap that precedes charge carrier relaxation and significant ionic motion and (ii) an instantaneous lattice potential symmetry change that represents the onset of the crystallographic phase transition through ionic motion on longer timescales. We discuss the interconnection between these two non-thermal pathways with particular focus on the meaning of the critical fluence of the PIPT in different types of experiments. Based on this, we conclude that the PIPT threshold identified in optical experiments is most probably determined by the excitation density required to drive the lattice potential change rather than the IMT. These considerations suggest that the IMT can be driven by weaker excitation, predicting a transiently metallic, monoclinic state of $\mathrm{VO}_{2}$ that is not stabilized by the non-thermal structural transition and, thus, decays on ultrafast timescales.
\end{abstract}

Keywords:

photoinduced phase transition, ultrafast dynamics, correlated phenomena, insulator-to-metal transition, vanadium oxides, femtosecond time-resolved spectroscopy

\section{Contents}

1 Introduction

1.1 The phase transition(s) in $\mathrm{VO}_{2}$ under equilibrium conditions $\ldots \ldots \ldots \ldots$

1.2 The phase transition(s) in $\mathrm{VO}_{2}$ after optical excitation . . . . . . . . . . . . . 5

2 Experimental

$2.1 \mathrm{VO}_{2}$ samples used in this work . . . . . . . . . . . . . . . . . . . . . 9

2.2 Time-resolved techniques: Optical and photoemission spectroscopy . . . . . . . . . . . . 9

3 Electronic structure and dynamics during the photoinduced insulator-to-metal transition 11

3.1 Electronic properties during the thermal transition . . . . . . . . . . . . . . . 11

3.2 Photoinduced changes to the electronic band structure . . . . . . . . . . . . . . . . 12

3.3 Ultrafast dynamics during the photinduced electronic transition . . . . . . . . . . . . . . . . . 13

3.4 Summary: Instantaneous band gap collapse in photoexcited $\mathrm{VO}_{2} \ldots \ldots \ldots$. . . . . . . . . . . 17

*Corresponding author: staehler@fhi-berlin.mpg.de 
4.1 Optical properties during the thermal transition . . . . . . . . . . . . . . . 18

4.2 Transient optical response in the low- and high-temperature phase after weak excitation . . . . . . . . 18

4.3 The PIPT threshold . . . . . . . . . . . . . . . . . . . . . . 21

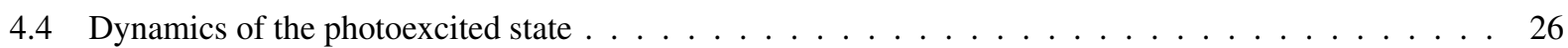

4.5 Summary: Quasi-instantaneous loss of potential symmetry in photoexcited $\mathrm{VO}_{2} \ldots \ldots$. . . . . . . . 30

5 Discussion: A comprehensive picture of the photoinduced, ultrafast phase transitions in $\mathrm{VO}_{2} \quad 30$

6 Summary and conclusions

7 Acknowledgements

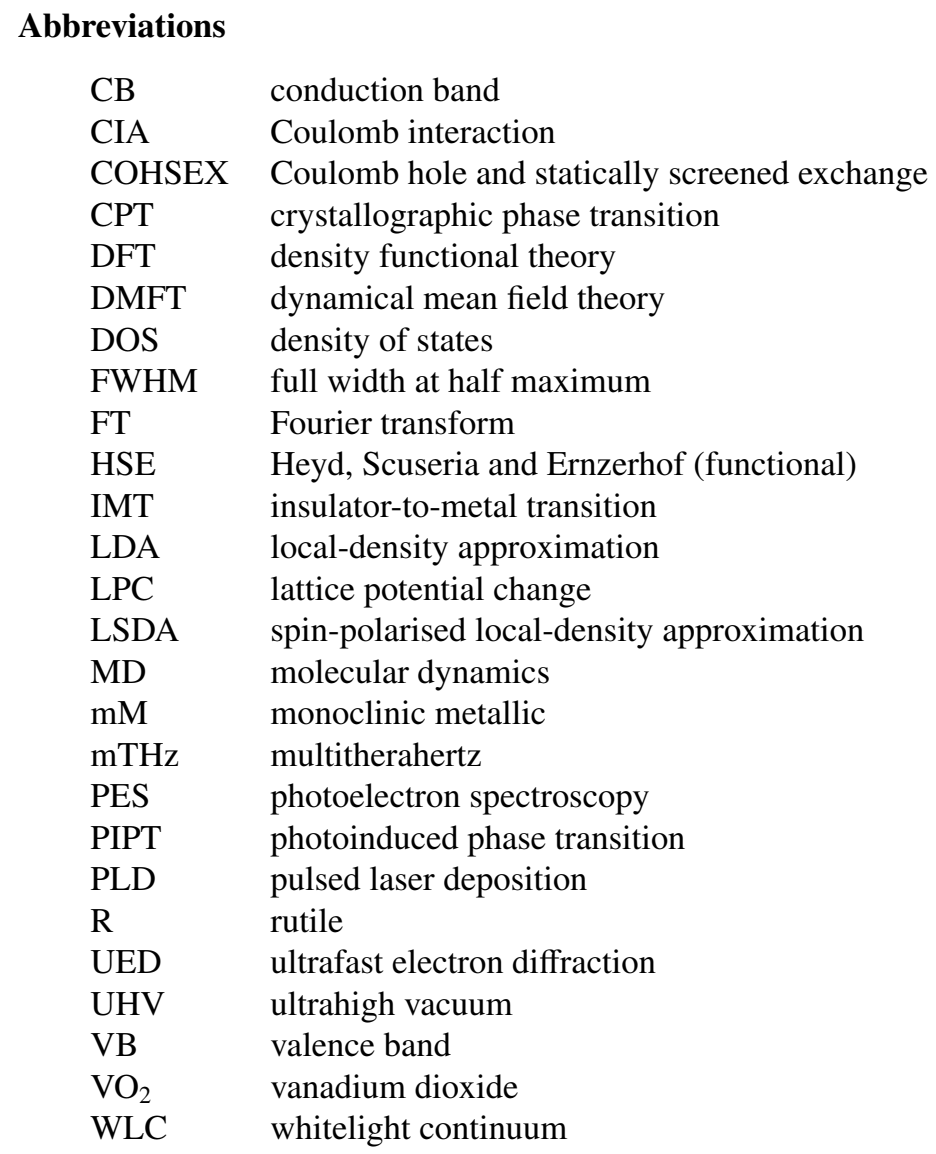

\section{Introduction}

Vanadium dioxide $\left(\mathrm{VO}_{2}\right)$ exhibits a thermally induced, first-order, reversible phase transition in crystallographic and electronic structure from an insulating-monoclinic to a metallic-rutile phase, both discovered more than 50 years ago by Westman and Morin [1,2]. Among other reasons, $\mathrm{VO}_{2}$ attracted significant scientific interest for many decades due to its transition temperature, which is only slightly above room temperature $\left(T_{\mathrm{C}} \simeq 340 \mathrm{~K}\right)$. The transitions are, thus, experimentally easily accessible and qualify $\mathrm{VO}_{2}$ for a manifold of applications. Another cause is the coincidence of the crystallographic phase transition (CPT) and the insulator-to-metal transition (IMT), which suggested a parental connection between the two. Not least the inability of both, theory and experiment, to unambiguously prove any suggested explanation of the underlying physical mechanisms provoked many scientists' curiousity. 
The establishment of ultrafast lasers in the second half of the last century as powerful tools to perform timeresolved spectroscopy opened up a new path to shed light into the controversy of the phase transition in $\mathrm{VO}_{2}$ when it was shown by Roach and Balberg [3] that the material exhibits a metal-like optical response after photoexcitation. The idea of the disentanglement of crystallographic and electronic contributions to the photoinduced phase transition (PIPT) of $\mathrm{VO}_{2}$ in the time domain was developed: Electronic processes were suspected to occur faster than ionic motion.

This article reviews our recent work on the PIPT of $\mathrm{VO}_{2}$ and relates it to selected, recent studies published on this matter. Without aiming at completeness, we attempt to draw a comprehensive picture of the dynamics launched when exciting vanadium dioxide optically. In this context, we show that sufficiently strong photoexcitation using $800 \mathrm{~nm}$ light leads to an instantaneous loss of the monoclinic potential symmetry and metallization of $\mathrm{VO}_{2}$ (on screening timescales) before significant ionic motion sets in $[4,5]$. In other words, we show that the lattice potential change (LPC) precedes the CPT, which is defined by the positions of the vanadium and oxygen atoms. The CPT occurs on later timescales [6] and its onset happens concurrently with hot carrier relaxation in the photoinduced-metallic band structure [5] that leads to distinct markers in the transient optical and THz response [7, 8]. We, furthermore, review the meaning of threshold fluences for the PIPTs in different time-resolved spectroscopic techniques and conclude that the frequently discussed critical excitation density extracted from optical data is most likely determined by the structural branch of the PIPT. Based on this, we propose the existence of a transiently metallic state of $\mathrm{VO}_{2}$ in its monoclinic lattice arrangement that, if accessible at lower fluences, should exhibit a comparably short lifetime determined by carrier relaxation dynamics only, because it is not stabilized by the non-thermal structural transition dynamics. In this context, we also assess the question of whether the distinction between Mott and Peierls mechanisms is at all applicable in this system.

\subsection{The phase transition( $(s)$ in $\mathrm{VO}_{2}$ under equilibrium conditions}

The lattice structures of $\mathrm{VO}_{2}$ for temperatures below and above $T_{\mathrm{C}}$ are displayed in Fig. 1, based on structural data from X-ray diffraction experiments $[9,10]$. The right panel shows two unit cells of the high temperature crystallographic structure, each containing 2 vanadium and 4 oxygen atoms. In this phase, the atoms arrange in a rutile habit, i.e. the $\mathrm{V}$ atoms, forming a tetragonal lattice, are surrounded by $\mathrm{O}$-octahedra (right). When lowering the temperature below $T_{\mathrm{C}}$, the vanadium atoms dimerise and the dimers tilt away from the rutile $c_{\mathrm{r}}$-axis (left). The oxygen octahedra are only slightly disturbed, while the vanadium atoms move by a larger amount. This structural change constitutes a lowering of symmetry and an effective doubling of the unit cell (the monoclinic unit cell contains 4 vanadium and 8 oxygen atoms). The volume changes by approx. $0.045 \%$ [11].

Beyond direct probes of the lattice structure of $\mathrm{VO}_{2}$ by diffraction techniques, both phases exhibit a distinct response in Raman spectroscopy: The insulating monoclinic phase (space group: P2 1 /c, No. 14) [12] has 18 Raman active phonon modes (at the $\Gamma$ point), as shown in $[13,14]$. In contrast, the rutile phase (space group: $\mathrm{P} 4_{2} / \mathrm{mnm}$, No. 136) exhibits only 4 Raman active modes, detected as very broad peaks by Raman spectroscopy [13].

Remarkably, at about the same $T_{\mathrm{C}}$, the conductivity of $\mathrm{VO}_{2}$ changes by up to five orders of magnitude [15], marking a metal-to-insulator transition. The phase transitions in $\mathrm{VO}_{2}$ are of first order due to the existence of latent heat and a diverging heat capacity [15]. Considering the atomic orbitals of $\mathrm{V}^{4+}$ and $\mathrm{O}^{2-}$ ions and the respective crystallographic structure, Goodenough gave a first description of the electronic structure in both phases [16]. As the central $\mathrm{V}$ atom sits inside an O-octahedron in the rutile phase, the $5 \mathrm{~V}-3 d$ orbitals are split by the octahedral crystal field into the usual $e_{g}$ and $t_{2 g}$ manifolds occupied by one electron ${ }^{1}$. Degeneracy of the $t_{2 g}$ manifold is lifted further and the $d_{x^{2}-y^{2}}$ orbital is split off at lower energies and named $d_{\|}{ }^{2}$, while the remaining $t_{2 g}$ orbitals are termed $\pi^{*}$. In the rutile phase, both bands overlap with the Fermi energy as depicted in Fig. 1 (right). This is different in the monoclinic phase where the displacement of the $\mathrm{V}$-atoms and their pairing along the rutile c-axis leads to a splitting of the $d_{\|}$band and a shift of $\pi^{*}$ to higher energies, as displayed in the left panel. Goodenough assumed that this splitting due to the structural distortion is sufficient to open up a band gap [16].

In contrast, Mott argued that, for a quantitatively correct description, electronic correlations have to be considered [17]. The Mott-Hubbard insulator model predicts a band gap between lower (occupied) and upper (empty) Hubbard

\footnotetext{
${ }^{1}$ Vanadium donates its two $4 s$ and two of the three $3 d$ electrons to the oxygen atoms, leading to occupied oxygen $2 p$ orbitals

${ }^{2} d_{\|}$is the orbital parallel to the rutile c-axis with strong direct overlap to the neighboring V atom. See [12] for an illustration.
} 


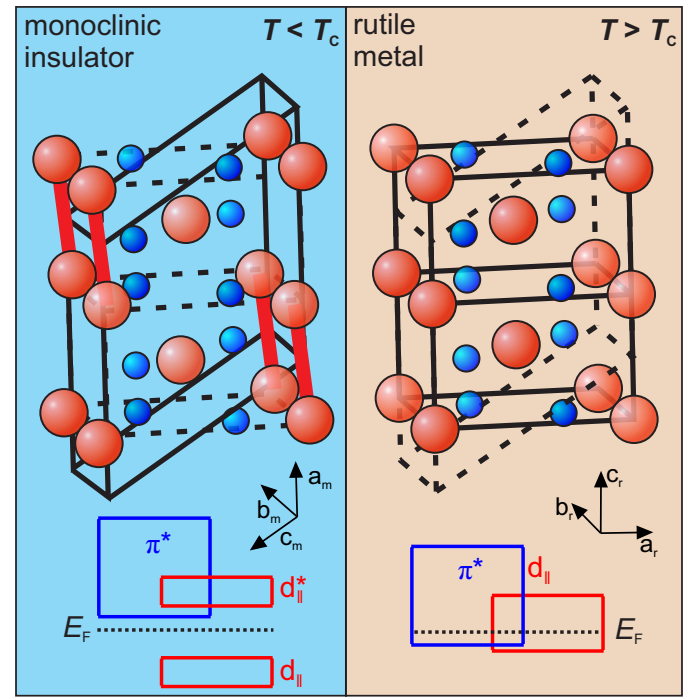

Figure 1: Comparison of monoclinic, insulating (left) and rutile metallic $\mathrm{VO}_{2}$ (right): Top: Crystallographic structures, the unit cells are indicated by the solid lines. Vanadium atoms are represented by the red balls, oxygen atoms are blue. Bottom: $\mathrm{Schematic}$ representation of the $\mathrm{VO}_{2}$ electronic band structure in the vicinity of the Fermi level, adopted from [12].

band of size $E_{\mathrm{G}}$, which depends on the bandwidths of the two Hubbard bands and the intra-atomic correlation energy $U$, which results from Coulomb repulsion [18]. According to Mott, the displacement of the V-atoms in the monoclinic phase only decreases the bandwidth of the d-bands. This narrowing makes the system susceptible to a discontinuous Mott transition. To clarify which of those two models, if either, yields an appropriate description of $\mathrm{VO}_{2}$, many theoretical and experimental studies were performed, one of which even explicitely asked " $\mathrm{VO}_{2}$ : Peierls or MottHubbard?" in its title [19].

For a long time, an explanation for both phases of $\mathrm{VO}_{2}$ could not be provided by a single theoretical framework. $A b$ initio molecular dynamics (MD) simulations based on density functional theory (DFT) in the local-density approximation (LDA) did yield correct crystallographic structures, but no electronic band gap in the monoclinic phase, even though the electronic structure (band overlap) around the Fermi energy is significantly altered [19]. The opposite failure, an insulating rutile and insulating monoclinic ground state was found when using DFT in the spin-polarised local-density approximation (LSDA) [20]. Choosing the 'right' hybrid functional (Heyd, Scuseria and Ernzerhof (HSE), including the exact electron-electron interaction to some extent) results in the correct insulating monoclinic and metallic rutile electronic structures [21], but concerns were raised that similar problems occur when including spin polarisation [22-24]: The rutile ground state becomes insulating and the monoclinic one magnetic.

With the improvements of theoretical methods and models, more consistent results were achieved recently: Simplified model $G W$ calculations $^{3}$ yield the correct electronic structure for both crystallographic phases [26]. Also, cluster dynamical mean field theory (DMFT) plus DFT+LDA including a parameter $U$ (constant for both phases) yields the right electronic structure in rutile and monoclinic phase [27]. Finally, DFT-LDA plus a self-consistent $G W$ correction on the COHSEX (Coulomb hole and statically screened exchange) level plus a one-step, perturbative $G_{0} W_{0}$ calculation ${ }^{4}$ also successfully describes both phases of $\mathrm{VO}_{2}[28,29]$. Recent calculations yield band gaps between 0.55 and $0.65 \mathrm{eV}$ of monoclinic $\mathrm{VO}_{2}$ and a plasma frequencies $\omega_{\mathrm{P}}$ between 1.5 and $2.9 \mathrm{eV}$ [27-31]. ${ }^{5}$

It seems that different theoretical approaches are able to describe the electronic properties of the monoclinic phase correctly when they a) accommodate for non-local electron-electron interaction and screening, but still on a single

\footnotetext{
${ }^{3}$ In Hedin's $G W$ approximation [25] the complex self energy $\Sigma$ of a quasiparticle is approximated by the product of the single particle Greens function $G$ and the screened Coulomb interaction $W$. In the model $G W$ the self energy only mimics this definition.

${ }^{4}$ Eigenvalues and orbitals are not calculated self consistently in this last step.

${ }^{5}$ The small size of the $\mathrm{VO}_{2}$ band gap at low temperatures is on the order of semiconductor energy gaps. Nevertheless, we will stick to the historical terminology of an insulating low-temperature phase in the following.
} 
quasiparticle band-structure level (moderate correlations) and b) use the monoclinic crystallographic structure. To our knowledge, it is widely agreed that strong electron correlation is not a sufficient condition to open an insulating gap in rutile $\mathrm{VO}_{2}$ or, in other words, the monoclinic structure is necessary to make $\mathrm{VO}_{2}$ insulating. Disaccord remained, however, until very recently, about the question whether the monoclinic structure also is a sufficient condition to open the insulating gap or if a monoclinic metallic $(\mathrm{mM})$ phase of $\mathrm{VO}_{2}$ exists. The observation of a $\mathrm{mM}$ phase in $\mathrm{VO}_{2}$ would, thus, unambiguously show that strong electron-electron interaction is necessary to form the insulating state. Strongly simplified, the current controversy boils down to the question of whether the insulator-to-metal transition occurs independent of the crystallographic transition (i.e. with the monoclinic structure preserved) or whether it is a consequence of the latter.

Experimentally, all (equilibrium) phases of $\mathrm{VO}_{2}$ have been characterized extensively during the last decades. For instance photoemission spectroscopy [32-35], Raman spectroscopy [13, 14] and diffraction techniques [9, 10] were used to either characterize the electronic or the crystallographic structure to name a few contributions of the vast amount of studies. In recent years, particular focus lay in the combination of structurally and electronically sensitive techniques or manipulation of the experimental conditions (e.g. pressure/strain) in order to study the relevance of a $\mathrm{mM}$ phase of $\mathrm{VO}_{2}$ during the thermal transition [36-41].

\subsection{The phase transition(s) in $\mathrm{VO}_{2}$ after optical excitation}

Another approach to disentangling lattice and elecronic contributions is to perform time-resolved out-of-equilibrium studies: The material is perturbed by a momentary stimulus and its progression towards equilibrium monitored by various types of techniques. Often, ultrashort laser pulses are used to, for example, access the timescales of electronelectron scattering and nuclear motions: The electric field of the incident photons interacts with the solid and induces a polarization. If photons are absorbed, electrons are redistributed and transiently occupy states that are empty under equilibrium conditions. The photoexcitation, if faster than the half-period of the phonon modes, may thereby also launch coherent lattice vibrations. Both, charge carriers and coherent phonons, will dissipate energy and eventually reach equilibrium with their environment. Importantly, both types of quasiparticles can interact with each other through scattering events with a phase space that is determined by the electronic and phononic band structures. To make matters more complicated, also these band structures may be modified by the photoexcitation, potentially leading to very complex non-equilibrium dynamics. The reason for this is that, in a solid, the electronic bands and crystallographic structure are determined by the interplay of the electrons and ions through the screened Coulomb interaction (CIA). The resulting forces define the potential energy surface and, thus, the (equilibrium) electronic levels and ionic arrangement. Strong photoexcitation can significantly change the screening of the CIA through the creation of quasi-free carriers. The change of screening alters the crystallographic potential of the solid and its electronic structure. Note that, if occuring on screening timescales of the free carriers, such changes to the potential usually happen on a femtosecond timescale [42-45], i.e. potentially within the duration of the exciting laser pulse and, in particular, before significant ionic motion occurs. For simplification, processes faster than the time resolution of the respective experiment are termed instantaneous in the following and their dynamics quantitatively discussed where necessary. The close relationship between the mechanisms dicussed above requires a clear disentanglement between the photoinduced charge redistribution, the concurrent lattice potential change and its effect on electronic and crystallographic structure.

Roach and Balberg [3] were the first to present experimental evidence for a photoinduced insulator-to-metal transition in $\mathrm{VO}_{2}$ after optical excitation with a pulsed laser. In particular, they showed that, after 20 ns, photoexcited $\mathrm{VO}_{2}$ exhibits a metal-like optical response, i.e. the photoexcitation has changed the dielectric function. These results promised that time-resolved spectroscopy (with improved time resolution) might eventually be able to elucidate the driving mechanism of the electronic phase transition.

Fig. 2 illustrates two possible pathways of the photoinduced insulator-to-metal transition in $\mathrm{VO}_{2}$. If electron correlations were responsible for the insulating gap, sufficiently strong photoexcitation, which - initially - occurs solely in the electronic system, could instantaneously disturb them by a change of the screened CIA, leading to an immediate collapse of the band gap, i.e. the IMT. In this case, the insulator-to-metal transition (IMT) should be driven by the screening through low-energy intraband transitions in partially occupied bands (typically on femtosecond timescales) followed by ionic motion driving the CPT. If a structural rearrangement or even the crystallographic phase transition (CPT) is required to close the band gap of $\mathrm{VO}_{2}$, the IMT will occur on timescales characteristic of ionic motion. 


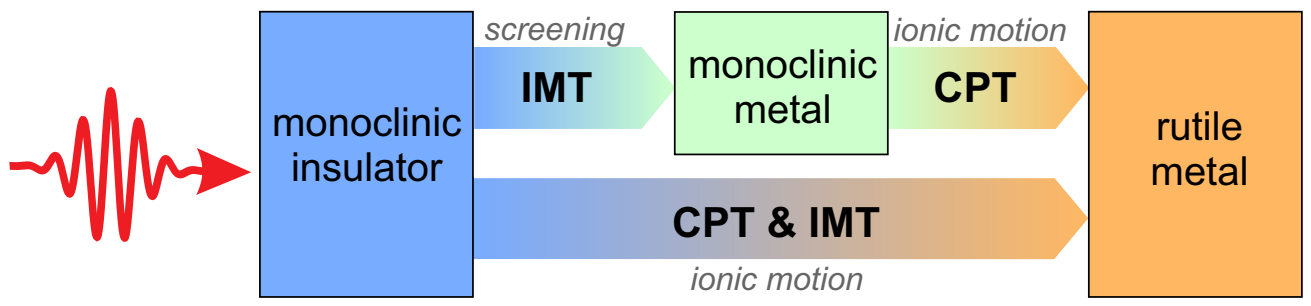

Figure 2: Possible pathways of the PIPT in $\mathrm{VO}_{2}$. If strong correlations in the electronic system are the origin of the insulating band gap, photoexcitation of monoclinic, insulating $\mathrm{VO}_{2}$ could lead to a sufficiently strong change of the screened Coulomb interaction causing the IMT before significant ionic motion occurs. This is different if the IMT occurs concurrently with structural rearrangement.

Pioneering work was done by Cavalleri et al. who used transient optical reflectivity and transmission with superior time resolution to show a threshold behaviour for the maximum speed in the optical response [46]. They demonstrated that the rise time of the optical signal was not limited by the duration of their broadband laser pulses, but amounted to $80 \mathrm{fs}$ at minimum, even using $15 \mathrm{fs}$ pump pulses. This observation of a structural bottleneck in the optical response was interpreted as a consequence of a lattice-mediated, Peierls-type mechanism. In this picture, the photoexcitation coherently excites a $6 \mathrm{THz}$ phonon that maps the monoclinic onto the rutile crystallographic structure, thereby driving the structural transition. It should be noted that, at the time, it was still unknown that the phonon mode along the V-dimers oscillates at $10 \mathrm{THz}$ and that tilting of the dimers happens at $6 \mathrm{THz}$ [47]. Neither of these modes alone maps the monoclinic onto the rutile structure; they are spectators of the CPT and, at the same time, do not exist in the hightemperature phase. As reflected by the impressive number of experimental follow-up works of different groups, the study by Cavalleri and coworkers motivated researchers to investigate the $\mathrm{VO}_{2}$ PIPT using time-resolved techniques that are able to selectively probe the lattice and electron dynamics: Optical spectroscopy simply does not purely probe electronic levels, but the whole dielectric function, including, for instance, contributions from the polarizability of the lattice. In addition, the lack of an absolute energy scale makes it very difficult to distinguish between free carriers within the conduction and valence band (of the insulating band structure) due to photoexcitation and a truly metallic response of a system due to a band gap collapse and finite density of states at the Fermi energy.

The group of Leitenstorfer et al. was the first to investigate the structural and electronic phase transition in photoexcited $\mathrm{VO}_{2}$ simultaneously $[8,48]$. They used time-resolved multiterahertz $(\mathrm{mTHz})$ spectroscopy in a frequency window that allowed the observation of the phononic system ( $\mathrm{P}$ in Fig. 3a)) in the region of high-energy oxygen cage modes and the even higher lying electronic response (E). By excitation density-dependent measurements, they determined a phase transition threshold at a pump-probe delay of $1 \mathrm{ps}$ for the wavelength-integrated spectra, exposing a non-linearity in the $\mathrm{mTHz}$ response for a critical fluence of $\Phi_{C}=4.6 \mathrm{~mJ} / \mathrm{cm}^{2}$. In addition, the authors observed a coherent wave packet motion in the photoexcited state and an ultrafast onset of a metallic response (E) in the THz conductivity without evidence of a structural bottleneck (cf. Fig. 3b), c) and [49, 50]). Based on these observations, the dynamics caused by photoexcitation were discussed in a local picture $[8,48]$, focussing on the nuclear motion of the vanadium dimers, which had proven to be successful when describing the $\mathrm{VO}_{2}$ phase transition(s) using cluster extended DMFT [27]. Fig. 3d) from [48] illustrates how the, initially highly mobile, excited electrons localize in a transient state and are subject to vibrations of the V-V dimer. It was suggested that, during their relaxation, they pass a mobility edge similar to the one introduced by Anderson [51]. As this mobility edge depends on the excitation density, the persistent change of the electronic conductivity for excitation above the critical fluence $\Phi_{C}$ was explained by a drop of the mobility edge below the transiently occupied electronic states [8, 48].

Fig. 3f) and g) translate the above described, dimer-based illustration of the photoinduced processes into a simplified band diagram of the $\mathrm{VO}_{2}$ solid. While the electrons, for low fluences, rapidly relax to local states that do not contribute to a significantly enhanced $\mathrm{THz}$ conductivity, strong photoexcitation leads to carrier densities that are sufficiently large to form an electron-hole plasma. This occurs when the mean distance between the carriers is smaller than their Bohr radius. The so-called Mott density, at which this (de)localization occurs is not an exclusive property of strongly correlated electron systems, but also occurs in "ordinary" semiconductors like GaAs [43, 52] or ZnO [53, 54]. Once the critical fluence is achieved, the delocalized carriers form quasi-Fermi levels in the valence and conduction band (VB and CB, Fig. 3g)), respectively and can contribute to an enhanced optical conductivity. The increased num- 
ber of possible low-energy excitations (charge displacements) in the excited state also changes the screening of the CIA and may, therefore, lead to a renormalization of the band gap. At the same time, this free carrier plasma affects the lattice, as it is created by the depopulation of the bonding orbitals by photoexcitation (see [52] and references therein). Note that in polar semiconductors like GaAs and $\mathrm{ZnO}$, polaron formation, i.e. another form of strong charge carrier coupling to the lattice, can also occur $[55,56]$.
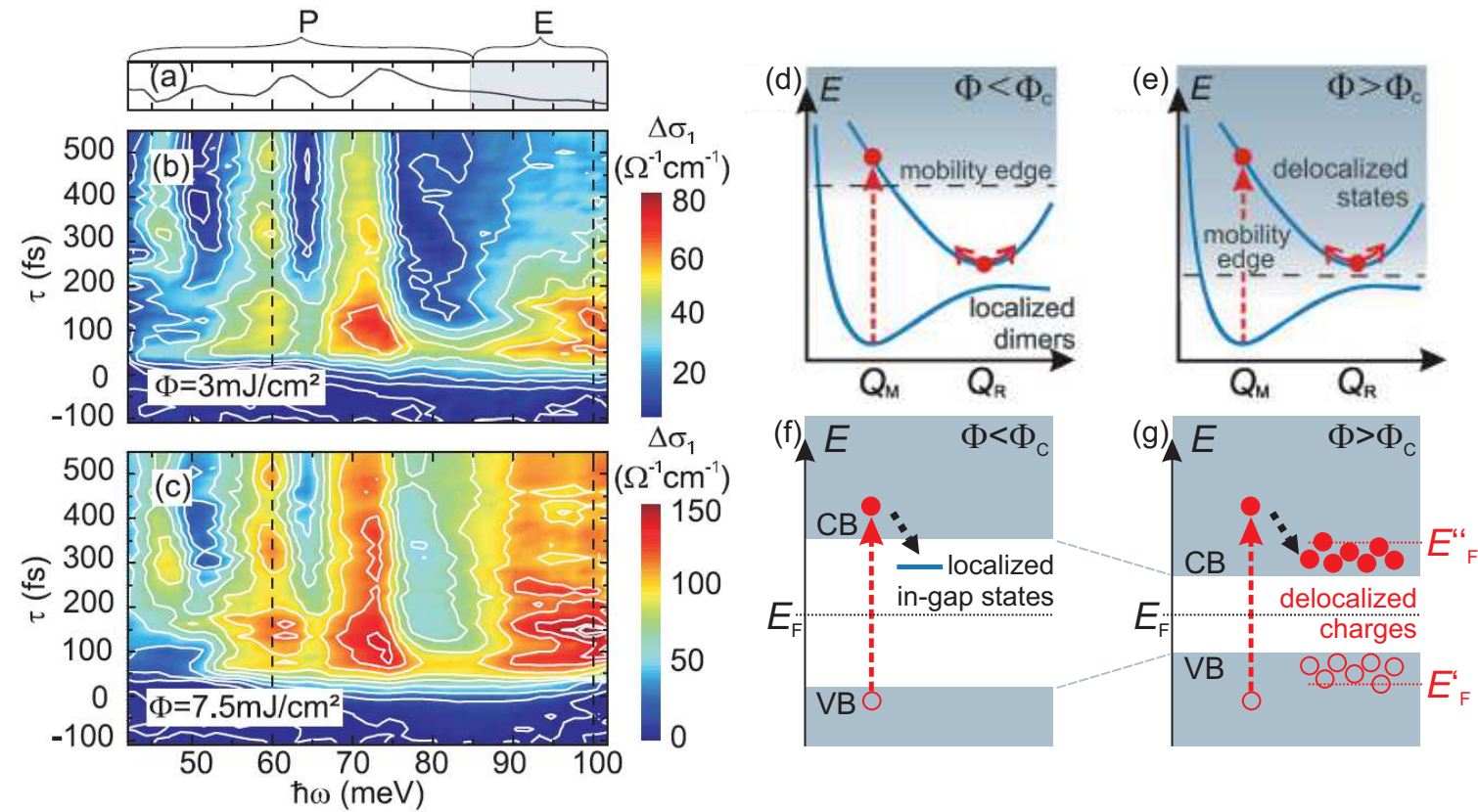

Figure 3: Probing the PIPT using multiterahertz (mTHz) spectroscopy. a) mTHz spectroscopy monitores the lattice dynamics (P) by resonant probing of the oxygen cage modes and the electronic response (E) at higher frequencies. b) and c) spectrally resolved, photoinduced change of the optical conductivity after below and above threshold pumping, respectively. a-c) adopted from [8]. d) and e) Local view of the photoinduced dynamics in $\mathrm{VO}_{2}$, based on schematic energy surfaces as a function of dimer coordinate $Q$ for weak and strong excitation, respectively. Adopted from [48]. f) and g) Schematic translation of d) and e) to a band structure-based perspective.

In order to monitor the CPT in real time, various types of diffraction experiments were performed in a timeresolved scheme. These studies show that the change of the ionic positions happens on multiple timescales from the sub-ps regime, extending to ns and following different steps of atomic rearrangements until the atoms resemble the equilibrium high-temperature structure [6, 57-60]. Unfortunately, the initial ultrafast timescale (sub-100 fs), which would allow to monitor the onset of the CPT, was not accessible in these studies due to the lack of time resolution. However, in the most recent work by Siwick et al. [6], significant progress towards a complete understanding of the structural transition was achieved: Using ultrafast electron diffraction (UED) with significantly improved time resolution (160 fs) the group unveiled two threshold fluences that expose an intermediate fluence regime between 2 and $9 \mathrm{~mJ} / \mathrm{cm}^{2}$ where a comparably slow $(1.6 \pm 0.2 \mathrm{ps})$ reorganization in the valence charge density of monoclinic $\mathrm{VO}_{2}$ is suggested, i.e. in the absence of a photoinduced CPT. Above $9 \mathrm{~mJ} / \mathrm{cm}^{2}$, a non-thermal melting $(300 \pm 160 \mathrm{fs}) \mathrm{of}$ the V-V dimerization is observed that is extrapolated to saturate for the complete probing volume at $43 \mathrm{~mJ} / \mathrm{cm}^{2}$, i.e. at 4-5 times $\Phi_{C}[6] .{ }^{6}$ Besides the UED, the authors also present fluence-dependent, time-resolved IR transmittivity data that exhibits a metal-like response for fluences below the CPT threshold fluence, suggesting that the electronic transition can occur independent of the un-pairing of the vanadium dimers.

\footnotetext{
${ }^{6}$ The ratio of saturation and threshold fluence is an important value, as it can be used as a benchmark when relating different types (optical, diffraction etc.) of fluence-dependent experiments with each other: Small errors in the spot size determination, losses in absorption/reflection at different angles of incidence, absorption properties, and inhomogeneities of the samples lead to uncertainties in the fluence determination. Moreover, different techniques show different probing volumes.
} 
A transient phase with monoclinic lattice structure exhibiting metallic signatures has also been reported in timeresolved studies by Ref.[37, 39, 40] and statically at high pressure ( $>10 \mathrm{GPa}$ ) by [38]. Also, very recently, pumpprobe microscopy combined with nanoimaging of $\mathrm{VO}_{2}$ unveiled a high susceptebility of the transient optical response of $\mathrm{VO}_{2}$ microcrystals to local variations of doping, defects, or strain [61]. All of these experiments, unfortunately, lack an absolute energy scale, as they are probing the dielectric function of the material optically. Highly excited matter can, as exemplified in Fig. 3g), show metallic character due to transiently free carriers that contribute to screening. Typical excitation densities for the PIPT on the order of $10 \%$ of the vanadium atoms (with one V $3 d$ electron each) lead to estimated ${ }^{7}$ frequencies of the non-equilibrium plasma of approximately one third of $\omega_{\mathrm{P}}$ of the equilibrium rutile metallic phase and, thus, to screening response times below $10 \mathrm{fs}$. A metal, however, in its simplest definition, is a material that exhibits finite density of states at its equilibrium Fermi energy $E_{\mathrm{F}}$ contrary to an excited semiconductor with metallic properties. Enhanced conductivity due to free carriers in VB and CB, as illustrated by the quasi-Fermi levels in Fig. 3g), is not an unambiguous proof of metallicity. In order to circumvent these difficulties, previous studies used time-resolved photoelectron spectroscopy (PES) to measure the change of the electronic structure of $\mathrm{VO}_{2}$ with an absolute energy scale $[62,63]$. Unfortunately, due to the too low repetition rate $(50 \mathrm{~Hz})$ of these experiments, inhibiting the required statistics [62] and the lack of sufficient time resolution ( $>150 \mathrm{fs}$ ), the dynamics of the IMT were inaccessible [63].

Despite many investigations focussing on the phase transitions of $\mathrm{VO}_{2}$, from which only a fraction was mentioned above, several open questions remain. The sequence of elementary processes during the photoinduced phase transition is still under debate: Is the detour via atomic motion after photoexcitation necessary to reach a metallic phase? Is the $80 \mathrm{fs}$ structural bottleneck in the optical response related to a real IMT caused by ionic rearrangement or is it simply a consequence of a change of the dielectric function due to ionic motion that is not accompanied by actual formation of a Fermi surface at the equilibrium $E_{F}$ ? What is the nature of the transient phase of $\mathrm{VO}_{2}$ before all components of the system thermalise? Is there a (transient) monoclinic metal? Or, in other words, is ionic motion a necessary condition for the IMT?

This article discusses complementary approaches based on time-resolved photoelectron and coherent phonon spectroscopy in order to shed light onto these questions. As mentioned further above, photoexcitation of a solid can, if sufficiently fast, launch coherent lattice vibrations. The phonon spectrum is determined by the lattice potential and can, thus, be used to probe changes to it in the vicinity of a phase transition [64]. Such changes can be reflected in phonon softening/hardening that result from optically driven changes to the potential curvature [65-70] or in a vanishing/occurence of modes due to a change of the potential symmetry of the system that can be accompanied by phonon softening towards zero frequency in a second order transition [67]. Time-resolved coherent phonon spectroscopy, thus, enables the investigation of the lattice potential change, which is the cause of the CPT. This study is complemented by time-resolved PES that allows to monitor the electronic structure and carrier dynamics in the region of the $\mathrm{VO}_{2}$ band gap around the Fermi energy on an absolute energy scale that enables unambigious measurement of the metallicity.

We will first demonstrate using time-resolved PES that the insulating gap of $\mathrm{VO}_{2}$ collapses quasi-instantaneously, without any lattice-related dynamics modifying the electronic structure at later times (Fig. 4, bottom). These results will be discussed in the framework of recent first-principles many body pertubation theory calculations from Rubio and coworkers [5] which show that the pure creation of holes at the top valence band are sufficient to destabilize the gap in the monoclinic structure. The transient phase of $\mathrm{VO}_{2}$ is, directly after photoexcitation, a monoclinic metal. We will then turn to the CPT, starting with a characterization of the (equilibrium) lattice potential of the monoclinic and rutile phase, respectively (Fig. 4, top) using time-resolved coherent phonon spectroscopy after weak photoexcitation above and below $T_{\mathrm{C}}$. It will then be shown that, in addition to the prompt metallization, strong photoexcitation also leads to an instantaneous loss of the initial monoclinic lattice potential symmetry [4, 7]. Despite this LPC the atoms are, according to recent diffraction experiments, still in their monoclinic positions (Fig. 4, center) [6]. These results give novel insight into the driving mechanism of the PIPT of $\mathrm{VO}_{2}$ and the properties of the transient phase: An instantaneous metallisation is observed, as a direct consequence of the charge redistribution through photoexcitation. The latter changes the screening of the Coulomb interaction. The photoinduced, transient phase is metallic with the monoclinic real space lattice structure, while the lattice potential is already raised above the monoclinic symmetry.

${ }^{7}$ Comparable effective masses are assumed. 
Combined with the most recent experimental works of other groups $[6,48,61,71]$, these findings sketch a comprehensive view of the photoinduced phase transitions in $\mathrm{VO}_{2}$ when excited above the critical fluence. We, furthermore, discuss the meaning of the different critical fluences determined by different experimental techniques, extracted by the fluence dependency of different observables. It seems highly likely that the critical fluence determined by optical spectroscopy corresponds to an excitation density that is strong enough to launch the LPC, i.e. the initiation of the CPT, which is not necessarily connected to the fluence needed to drive the IMT. In this sense, a transiently metallic state of monoclinic $\mathrm{VO}_{2}$ could be accessible at low excitation densities, which is not stabilized by a concurrent structural transition and which has a lifetime that is dictated by the timescales of carrier relaxation.

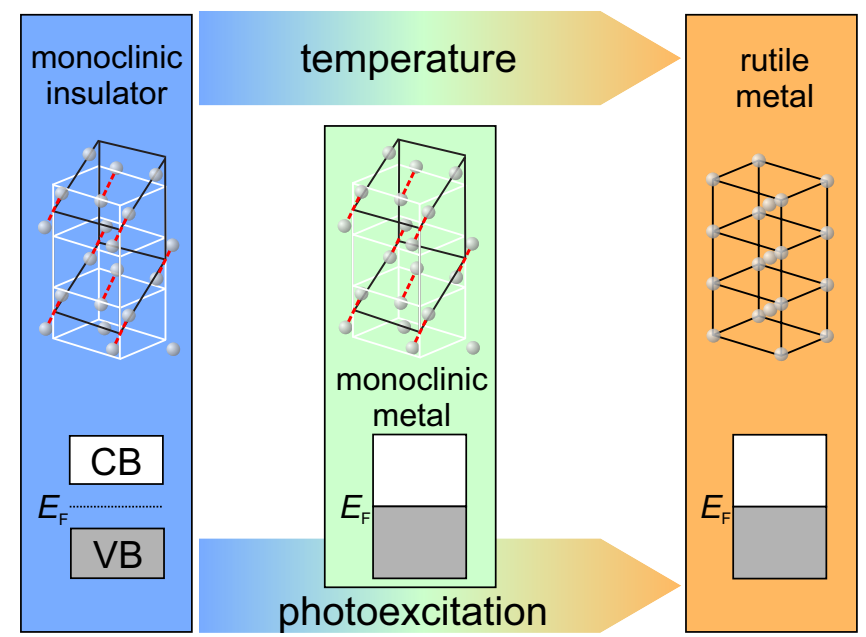

Figure 4: Selected equilibrium and non-equilibrium phases of vanadium dioxide. Weak photoexcitation enables the characterization of the thermal monoclinic and rutile phase, while strong excitation eventually drives $\mathrm{VO}_{2}$ into its high-temperature phase. The transient phase of $\mathrm{VO}_{2}$ is metallic and its lattice potential has lost the monoclinic symmetry despite monoclinic ionic positions.

\section{Experimental}

\section{1. $\mathrm{VO}_{2}$ samples used in this work}

Vanadiumoxides exist in many stoichiometries and mixed oxidation states [72]. The $\mathrm{VO}_{2}$ thin film samples used here were grown in the group of Richard Haglund (Vanderbilt University). For the optical spectroscopy experiments, a $200 \mathrm{~nm} \mathrm{VO}_{2}$ thin film was grown by pulsed laser deposition (PLD) on $n$-doped $\mathrm{Si}(100)$ [73], resulting in a polycrystalline non-epitaxial film. The sample used for photoelectron spectroscopy (PES) was $45 \mathrm{~nm}$ thick, room-temperature-PLD-grown on c-cut sapphire $\left(\mathrm{Al}_{2} \mathrm{O}_{3}(0001)\right.$ surface), leading to a smooth polycrystalline thin film, required for surface-sensitive PES [74, 75]. It was mounted inside the ultra-high vacuum (UHV) chamber in a Tantalum-pocket that provides electrical contact to the $\mathrm{VO}_{2}$ film, which is necessary to avoid sample charging during the PES experiments. Before each set of photoemission measurements, the sample was, for 30 minutes, annealed at $600 \mathrm{~K}$ in an oxygen atmosphere $\left(\mathrm{O}_{2}\right.$ pressure of $10^{-4}$ mbar) to generate a clean surface, while maintaining the correct stoichiometry of $\mathrm{VO}_{2}$. The surface quality was checked by monitoring charging effects and in situ detection of the characteristic hysteresis loop (see section 3.1) and compared to literature [73, 76-79]. Fig. 5 exemplarily shows the reflectivity change at $800 \mathrm{~nm}$ that exhibits a hysteresis loop with the slightly displaced heating and cooling branches (orange, blue), both showing a significant and consistent change in reflectivity at the phase transition temperature.

\subsection{Time-resolved techniques: Optical and photoemission spectroscopy}

Fig. 6a) displays the experimental pump-probe scheme, in which both, time-resolved optical and photoemission spectroscopy, were conducted. First, a pump pulse (red) perturbs the sample. It is followed by a second, time-delayed, probe pulse (blue) that subsequently probes the properties of the sample. By variation of the time delay between both 


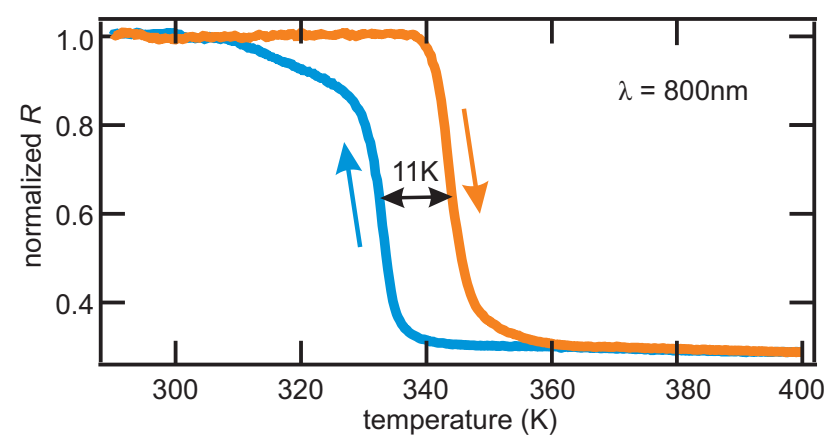

Figure 5: The reflectivity of $\mathrm{VO}_{2}$ shows a typical hysteresis loop when heating and cooling across the phase transition temperature. A sudden drop in reflectivity at $800 \mathrm{~nm}$ is observed when crossing the insulator-metal transition.

a)

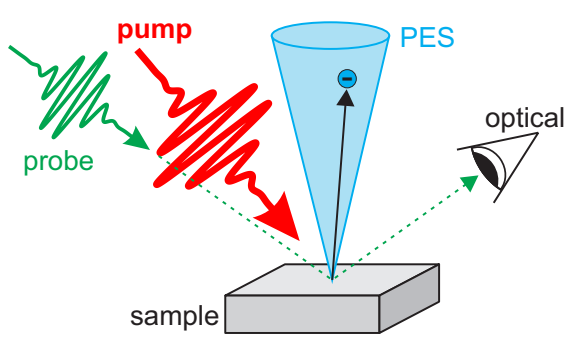

b)

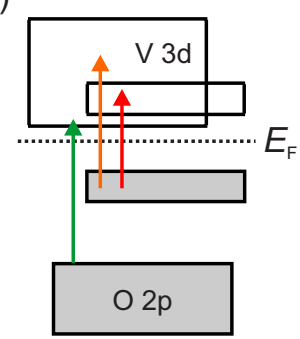

c)

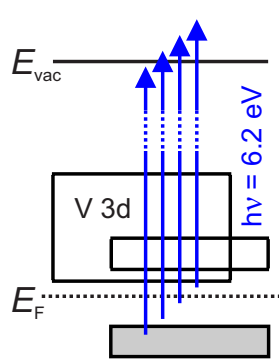

Figure 6: Experimental scheme: a) Illustration of time-resolved experiments using subsequent pump (red) and probe (green) laser pulses. The probed quantity is either the transient change in reflectivity or photoelectrons that are emitted from the sample. b) Illustration of exemplary transitions, simultaneously probed by broadband laser pulses. c) Photoemission probe of electrons in the vicinity of the insulating band gap by UV light.

pulses, the evolution of these properties can be measured in real time. The fundamental output $(\lambda=800 \mathrm{~nm}, 40 \mathrm{fs})$ of a commercial regenerative amplifier system (COHERENT RegA) served as the pump, providing a wide range of pulse energies to tune across the phase transition fluence threshold. The direct $d-d$ transitions induced by this photon energy $(h v=1.55 \mathrm{eV})$ are optically allowed due to the strong hybridization in the system.

Depending on the experiment, different probe photon energies were used for spectroscopy: In the optical spectroscopy experiments, fundamental $800 \mathrm{~nm}$ or broadband whitelight continuum (WLC) pulses, compressed close to their transform limit [80], monitored ultrafast changes of the dielectric function of the sample over a wide range of transitions significantly larger than the indirect band gap $(0.55-0.65 \mathrm{eV}$, see e.g. [28-30]) including transitions within the $3 d$ manifold and from the occupied $\mathrm{O} 2 p$ levels, as illustrated in Fig. 6b). The reflected probe-light intensity was recorded with a photodiode and lock-in amplifier (single color) or an optical spectrometer (broadband).

In the time-resolved PES experiment, $\mathrm{h} v=6.2 \mathrm{eV}$ pulses were generated from the fundamental amplifier output via a two-stage noncollinear SFG process (similar to [81]) to photoemit electrons from within the band gap region of insulating $\mathrm{VO}_{2}$. Fig. $6 \mathrm{c}$ ) illustrates this photoemission process. Detection of the photoelectrons using a hemispherical electron energy analyzer (SPECS Phoibos 100) allows the direct determination of the initial state energy, which enables the explicit measurement of the transient changes of the $\mathrm{VO}_{2}$ band structure after photoexcitation. It should, nevertheless, be noted that time-dependent photoemission studies of $\mathrm{VO}_{2}$ are notoriously difficult (see also Refs. [62, 63]): Average heating of the probed sample region by the pump pulses has been reduced by using a comparably low repetition rate of $40 \mathrm{kHz}$ and cooling via the cryostat. However, this effect still set an upper limit to the pump fluence, as strong cooling goes along with another unwanted side effect: The reduced temperature decreases the conductivity of the sample as a whole, making the experiment susceptible to charging-related artifacts that can influence the energetic position of the photoelectron spectra. Vacuum space charge effects, due to a too large amount of photoemitted electrons per laser shot were omitted by choosing sufficiently low fluences for pumping (multiple- 
photon-photoemission) and probing, further limiting the range of applicable excitation fluences. It was reduced to excitation densities just above the phase transition threshold, as compared to (optical) experiments under ambient conditions. ${ }^{8}$ However, as discussed in detail in chapters 3 and 5, the inability to perform experiments over a wide range of fluences in time-resolved PES is not problematic, as this techniques directly measures the electronic structure in the band gap region. The energy of the photoelectron spectrum was routinely referenced to the Fermi edges of the high-temperature metallic phase and of the metallic sample holder. The latter was was in good electrical contact with the sample and its Fermi energy coincided with the one of metallic $\mathrm{VO}_{2}$. As the work function of the sample is affected by both, the thermal and the photoinduced transition and reflects an average over the distributions in the polycrystalline samples, its spectral signature, the low energy cut-off of the photoemission spectra, was not used for energy referencing.

\section{Electronic structure and dynamics during the photoinduced insulator-to-metal transition}

In order to assess the ultrafast dynamics of the photoinduced electronic transition, we use time-resolved photoemission experiments which provide direct insight into the dynamics of electronic structure and charge carriers. First, the low- and high-temperature phase are characterized in thermodynamic equilibrium (section 3.1): Upon heating of the $\mathrm{VO}_{2}$ sample, density of states (DOS) appears at the Fermi energy, providing a solid indicator for the insulator-tometal transition. We show that the photoinduced change of photoelectron intensity, 1 ps after optical excitation, i.e. at the time delay at which the PIPT threshold was defined in previous studies, equally follows a Fermi-Dirac distribution function, demonstrating that - at this time delay - $\mathrm{VO}_{2}$ indeed is metallic (section 3.2). Analysis of the time-resolved data unveils a quasi-instantaneous appearance of density of states at the Fermi energy (in the band gap region of insulating $\mathrm{VO}_{2}$ ) after strong photoexcitation (section 3.3), before the hot carriers relax on a 200 fs timscale and, in particular, before significant ionic motion has occured. Concordantly, many body pertubation theory calculations from Rubio and coworkers show that pure charge redistribution and recalculation of the band structure in a frozen lattice yields a collapse of the $\mathrm{VO}_{2}$ band gap and identify the driving mechanism to be the generation of photoholes at the top of the valence band, which significantly changes the screening of the CIA.

\subsection{Electronic properties during the thermal transition}

Fig. 7a) and b) show energy diagrams that illustrate the photoemission process for an insulator and a metal, respectively. When a metallic sample with work function $\Phi$ is investigated using a photon energy h $v_{\text {probe }}>\Phi$, the occupied density of states (DOS, orange) is, in a first approximation (neglecting e.g. transition matrix elements and scattering processes), projected from its initial energy to the continuum of final states above the sample vacuum level $E_{\mathrm{vac}}$. The detected photoemission intensity is indicated by the grey curve and exhibits a low energy cut-off (also called secondary edge) at $E_{\mathrm{vac}}$, due to the sample vacuum level and a high energy cut-off (also called Fermi edge) at $E_{\mathrm{FK}}$, because initial states are occupied according to a Fermi-Dirac distribution centered at the Fermi energy $E_{\mathrm{F}}$. The DOS of an insulator (blue in Fig. 7a)) approaches zero above the valence band. In reality, the valence band maximum is often smeared out due to the presence of localized (defect) states in the band gap. If the sample is not too defective, there is no DOS at $E_{F}$. This means, in contrast to a metal, an insulator does not exhibit a Fermi surface.

In the case of $\mathrm{VO}_{2}$, photons of $h v=6.2 \mathrm{eV}$ probe weakly bound states (down to $\sim 600 \mathrm{meV}$ below $E_{F}$ ), as the work function is on the order of $5.6 \mathrm{eV}$. Fig. 7c) and e) show photoemission spectra of $\mathrm{VO}_{2}$ upon heating and cooling, respectively. The highlighted spectra for the insulating (blue, $299 \mathrm{~K}$ ) and metallic (orange, $372 \mathrm{~K}$ ) phase clearly differ: While, in the low temperature phase, the PE intensity is close to zero at $E-E_{\mathrm{F}}=0$, the high temperature phase exhibits a high photoemission intensity at this energy. When plotted as a function of temperature (Fig. 7d)), the spectral weight below the Fermi energy follows a hysteresis centered at $330 \mathrm{~K}$, in agreement with the optical characterization of the sample (not shown, see Ref. [82]). The thermally induced change due to heating of the sample from $299 \mathrm{~K}$ to $372 \mathrm{~K}$, i.e. the difference between the orange and the blue spectrum, is depicted in Fig. 8b) (green). It clearly resembles the shape of a Fermi-Dirac distribution at $372 \mathrm{~K}$, convoluted with the spectral resolution of $90 \mathrm{meV}$, plotted in black for comparison. Heating $\mathrm{VO}_{2}$ above the critical temperature, thus, leads to the formation of additional DOS in the energy

\footnotetext{
${ }^{8}$ Much lower excitation densities were also not feasible, due the decrease of signal, leading to a poor signal-to-noise ratio.
} 

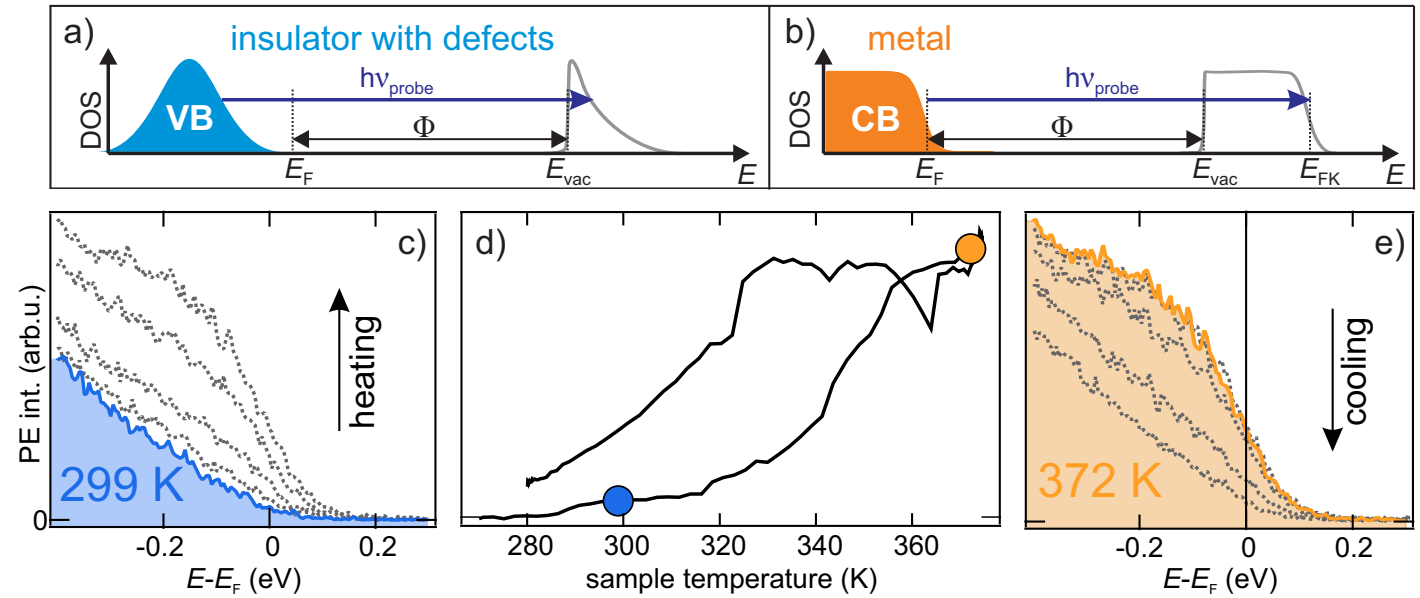

Figure 7: a) and b) illustrate the single-photon photoemission process and resulting photoelectron spectra for metallic and insulating sample (with defects), respectively. Modified from [82]. c) and e) PES spectra of $\mathrm{VO}_{2}$ using $6.2 \mathrm{eV}$ photons during heating and cooling of the sample between $299 \mathrm{~K}$ (blue) and $372 \mathrm{~K}$ (orange), respectively. Clearly, metallic DOS builds up in the band gap with increasing temperature. d) Integration of the $\mathrm{PE}$ intensity between -0.2 and $0 \mathrm{eV}$ as a function of temperature yields a hysteresis, showing that the experiment is sensitive to the electronic phase transition of $\mathrm{VO}_{2}$. Modified from [5]

region of the band gap of the insulating phase up to the Fermi energy, and the occupation distribution of these states is consistent with Fermi-Dirac statistics. In other words, a Fermi surface is formed.

The above analysis shows that the integrated photoemission intensity below the Fermi energy in our experiment is directly sensitive to the thermally driven IMT and, therefore, can be used as a marker for the latter. We would like to emphasize that the occurence of DOS in the gap region, i.e. the developement of a Fermi surface, is - in contrast to the change in reflectivity observed in the optical experiments in section 4 - an unambiguous probe of metallic $\mathrm{VO}_{2}$.

\subsection{Photoinduced changes to the electronic band structure}

After the characterisation of the equilibrium insulating and metallic phase of $\mathrm{VO}_{2}$ using PES, this section focusses on the photoinduced changes to the electronic structure of $\mathrm{VO}_{2}$. As a starting point, we analyse the photoinduced change at a time delay $\tau>1 \mathrm{ps}$, as this was used for PIPT threshold determination in previous studies [7, 8, 48]. To quantify the photoinduced changes, averaged photoelectron spectra before time zero and for $t>1 \mathrm{ps}^{9}$ after photoexcitation using an incindent fluence of $F=6.7 \pm 0.8 \mathrm{~mJ} / \mathrm{cm}^{-2}$, corresponding to an excitation density of $8 \%$ of the $\mathrm{V}$ atoms, are shown in Fig. 8a). This fluence lies above the threshold fluence determined optically [7, 83], but clearly below the saturation fluence as outlined in more detail in section 4.2. ${ }^{10}$ This means that only parts of the excited volume are transformed by the photoexcitation (see inset in Fig. 8a)). We, thus, estimate from previous optical studies at comparable fluences $[7,8,84]$ photoinduced changes to the spectral function on the order of a few percent of the thermally induced change.

Comparison of the two spectra before and after photoexcitation shows that neither exhibits the characteristic shape of a Fermi-distribution function, but the onset of the valence band of insulating $\mathrm{VO}_{2}$ and a secondary electron background created by the pump-light alone (non-zero intensity above the Fermi energy). The photoinduced change, i.e. the difference of the two curves in a), is displayed in Fig. 8b) (red curve). Its spectral shape compares very well with the thermally induced change (green) introduced above. The amplitude of the photoinduced change is, as expected, 20 times smaller than the one of the thermally induced spectrum. As, in this representation, only the additional, photoinduced photoelectrons in the region of the insulating gap are measured and because their spectral function coincides

\footnotetext{
${ }^{9}$ As will be shown in the next section, the PE spectra do not change for $t>1 \mathrm{ps}$. They were, therefore, integrated to improve the signal-to-noise ratio.

${ }^{10}$ As discussed in section 2, the fluence range feasible for PES is much smaller than for the optical experiments, as heat dissipation is more difficult under the required UHV conditions and effects like charging and multiphoton photoemission by the strong pump pulse need to be avoided.
} 


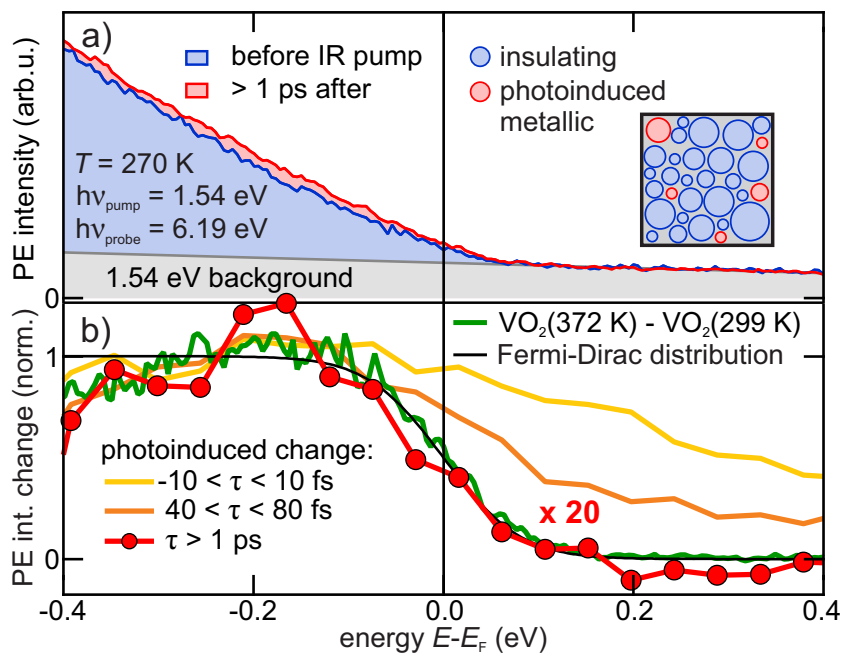

Figure 8: Photoinduced changes of the spectral function probed by time-resolved PES: a) Averaged PE spectra before (blue, -20...- 1 ps) and after photoexcitation (red, $1 \ldots 100 \mathrm{ps}$ ) with a fluence of $6.7 \mathrm{~mJ} / \mathrm{cm}^{2}$ and at a sample temperature of $270 \mathrm{~K}$. The inset illustrates how photoexcitation only switches selected domains. b) Subtraction yields the photoinduced change of photoemission intensity (red markers, data binned with $\Delta E=$ $45 \mathrm{meV}$ ), which resembles the thermally induced change (green). Both agree very well with a Fermi-Dirac distribution function (black) at $372 \mathrm{~K}$ broadened by the experimental resolution $(90 \mathrm{meV})$. At earlier delays (yellow and orange) the spectra clearly deviate.

with what was identified to be metallic $\mathrm{VO}_{2}$ in the previous section, we conclude that the photoinduced change to the spectral function results from an ultrafast metallisation of parts of the pumped region. Furthermore, it is a direct observation of the photoinduced insulator-to-metal transition in $\mathrm{VO}_{2}$.

These results verify that - if sufficiently strongly photoexcited $-\mathrm{VO}_{2}$ is metallic $1 \mathrm{ps}$ after the pump pulse is absorbed. At this time delay, the ions are already on their passage to their new quasi-equilibrium positions [6, 57]. The observation of metallic $\mathrm{VO}_{2}$ at this time delay, thus, does not carry any information about whether or not this metallization is a consequence of ionic motion or if the photoinduced disturbance of the electronic system would be sufficient to drive the IMT between 0 and 1 ps.

The preceding sections provide clear evidence that the performed photoemission experiments are sensitive to both the temperature- and photoinduced insulator-to-metal transition in $\mathrm{VO}_{2}$. In both cases, the metallisation manifests itself by additional density of states below the Fermi energy exhibiting the shape of a Fermi-Dirac distribution function. Whether the IMT transition occurs faster or simultaneously with the onset of the CPT can only be assessed by experiments on relevant timescales as discussed in the following.

\subsection{Ultrafast dynamics during the photinduced electronic transition}

If the driving mechanism of the IMT were ionic motion, the characteristic time, at which the gap-closing occurs $\left(\tau_{\text {gap }}\right)$, should coincide with a timescale that is characteristic of changes of the lattice $\left(\tau_{\text {lattice }}\right)$, for example the structural bottleneck timescale of $80 \mathrm{fs}$, reported in [46] (see Section 1.1). If, in contrast, a transient metallic phase is generated due to the disturbance of the electronic system through photoexcitation alone, while the atoms are still in their monoclinic positions, $\tau_{\text {gap }}$ should be shorter (on screening timescales of the quasi-free carriers) and the gap collapse should, thus, occur faster than the lattice change $\left(\tau_{\text {gap }}<\tau_{\text {lattice }}\right)$. Hot electron decay within the band gap energy region would, in both cases, only be observed when the gap is already closed. The thermal rutile (R), metallic phase is only reached after the whole system has thermalised and the ions have moved to their new quasi-equilibrium positions.

A typical time- and energy-resolved PES dataset is shown in Fig. 9a) in reference to the schematic band structure of insulating $\mathrm{VO}_{2}$ (on the right, energy axis is to scale). The equilibrium background has been extracted at negative pump-probe delays and subtracted from the data. Therefore, only the pump-induced change in photoemission intensity is displayed. This means that all photoelectrons extracted from this energy region contribute to photoexcited in-gap dynamics. At zero time delay between pump and probe pulse an intensity increase (red) is observed at all energies 


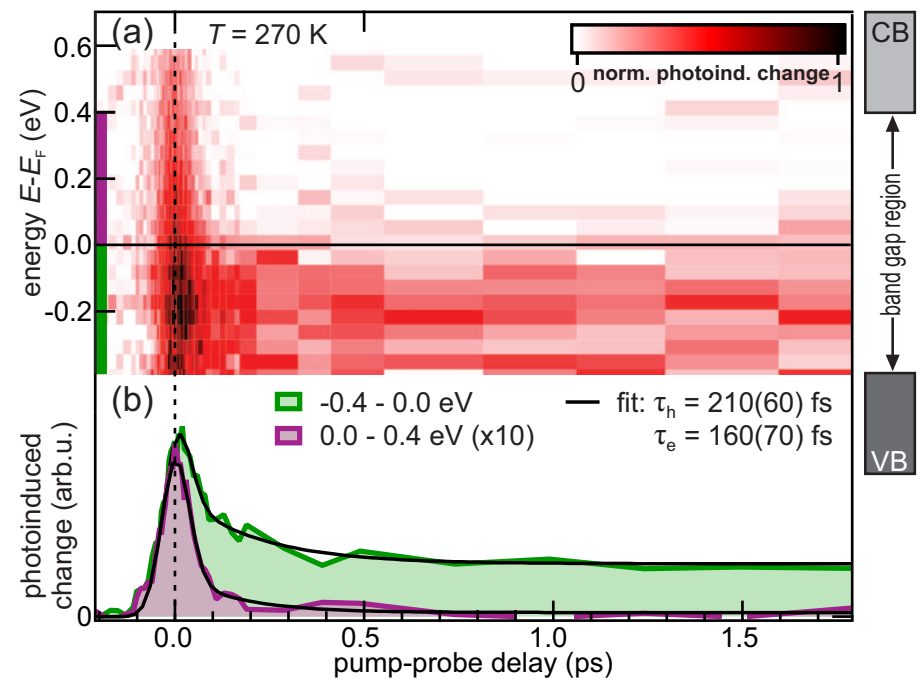

Figure 9: a) Photoinduced change of photoemission intensity induced by a $F=6.7 \mathrm{~mJ} / \mathrm{cm}^{2}$ pump pulse, centred at 1.54 eV photon energy, probed by UV $(h v=6.19 \mathrm{eV})$ light. The signal at delays smaller than $-1 \mathrm{ps}$ were used for background subtraction of the uncorrelated signal. The band scheme of insulating $\mathrm{VO}_{2}$ is shown for energy reference on the right. b) Integration of the time-dependent PE intensity above (purple) and below (green) $E_{\mathrm{F}}$ exposes the averaged hot electron and hole dynamics, respectively. Black curves are phenomenological, single exponential fits to the data (see text for details). Modified from [5].

between -0.4 and $0.6 \mathrm{meV}$ with respect to the Fermi energy. At positive pump-probe delays a photoinduced increase is detected below the Fermi energy, which persists unchanged for at least hundreds of picoseconds (not shown).

The main part of the intensity around time zero is interpreted as two-photon photoemission via virtual intermediate states in the band gap of unswitched, insulating $\mathrm{VO}_{2}$, as observed in other systems previously [85, 86]. The region between 400 and $600 \mathrm{meV}$ above the Fermi energy is used to extract the cross correlation of pump and probe pulse. Fitted with a Gaussian function (not shown) it yields a cross correlation width (FWHM) on the order of 90 fs, i.e. an average pulse duration of $61 \pm 5 \mathrm{fs}$ (see [82] for details). Integration of the photoinduced change of photoelectron intensity above (purple) and below the Fermi energy (green) yields the transients depicted in Fig. 9b). A contribution from two-photon photoemission via virtual intermediate states, exhibiting no lifetime, results in a peak at timezero in both traces. These peaks appear asymmetric, as they are superimposed by a decaying intensity. Below $E_{\mathrm{F}}$, the intensity evolves to a persisting increase at positive delays; above $E_{\mathrm{F}}$, this intensity decays to zero.

As discussed in the previous section, the additional PE intensity below the Fermi energy results from metallic patches of $\mathrm{VO}_{2}$ that have been switched by the optical excitation. The photoinduced PE intensity above the Fermi energy also shows up in the region of the band gap of insulating $\mathrm{VO}_{2}$. We, therefore, conclude that it results from photoexcited electrons that decay towards $E_{\mathrm{F}}$ in the metallic parts of the sample. A first quantification of the initial fast dynamics can be achieved by fitting single exponential decays to the data (black). The fit function also exhibits a constant offset and a delta function at time zero to account for the two-photon photoemission via virtual states. All contributions are convoluted with the laser pulses' envelope extracted at high energies. The time constants resulting from these fits $\left(\tau_{e}=160 \pm 70\right.$ fs and $\tau_{h}=210 \pm 60$ fs for the above and below $E_{\mathrm{F}}$ transient, respectively) represent mean electron and hole relaxation times, averaged over a limited energy and momentum window arond $E_{\mathrm{F}}$ and $\bar{\Gamma}$.

In order to allow carrier relaxation at energies of the insulating band gap (compare energy scheme on the right), it must have collapsed before the dynamics can occur, i.e. faster than $200 \mathrm{fs}$. Qualitatively, it must occur on timescales comparable or shorter than the pulse duration of $61 \mathrm{fs}$, as the PE spectra exhibit intensity in the band gap region from time zero on. A quantitative determination of the time needed for the band gap collapse can be achieved by a fit model. Rate equations describing the appearance of DOS and, on top of that, quasiparticle relaxation dynamics turned out to be too complex (inhomogeneous quadratic differential equation with at least one time-dependent coefficient) to yield a robust fit function. Therefore, a phenomenological model is used to quantify the timescales of the observed 


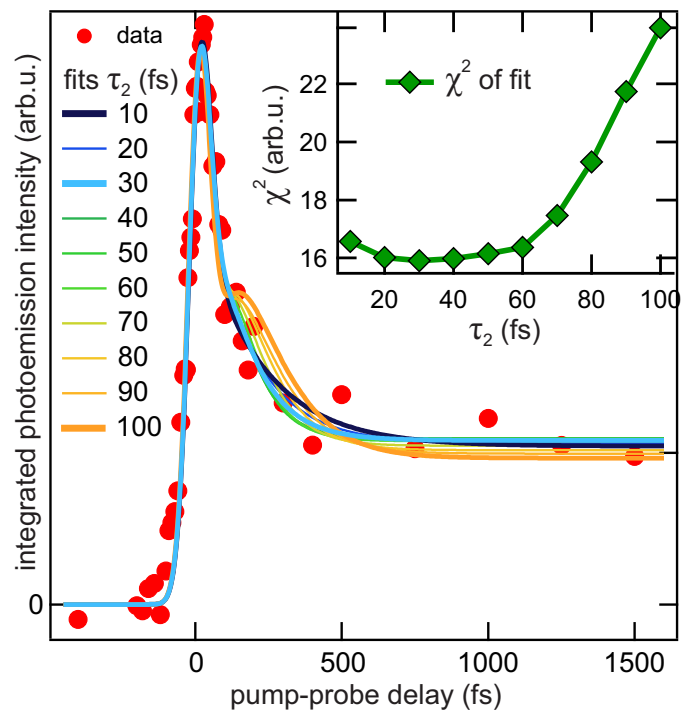

Figure 10: Exemplary fit results for transient metallisation of $\mathrm{VO}_{2}$ below the Fermi energy: Fits for various fixed rise times $\tau_{2}$. The inset shows the $\chi^{2}$-property of the fit with respect to the different values of $\tau_{2}$.

dynamics. The function $R(t)$ used to describe the PES response upon photoexcitation is:

$$
R(t)=\theta(t)\left(A_{1} e^{-t / \tau_{1}}-A_{2} e^{-t / \tau_{2}}+C\right)+D \cdot \delta(t)
$$

It consists of an exponential rise with amplitude $A_{2}$ and time constant $\tau_{2}$, an exponential decay with amplitude $A_{1}$ and time constant $\tau_{1}$ and a constant offset $C=A_{2}-A_{1}$ that fulfils the initial condition $R(0)=0$. The contribution of two-photon photoemission via virtual intermediate states is modelled as an approximate delta-distribution $\delta(t)$ with "amplitude" $D$. The Heaviside function $\theta(t)$ ensures zero response before time zero. The response $R(t)$ is convolved with the laser pulses envelope, and the resulting curve is then fitted to the data by a least-mean-squares algorithm.

The global fit optimum is reached for $\tau_{1}=125 \pm 74 \mathrm{fs}$ for the decay and $\tau_{2}=28 \pm 19 \mathrm{fs}$ for the rise. As the large error bars already indicate, the fit is not entirely robust when none of the parameters in $R(t)$ is kept constant. The reason is, that for small $\delta \tau=\tau_{2}-\tau_{1}$ the parameters, especially the two amplitudes, are not independent anymore.

To achieve an upper limit for the rise time, a trial and error method was used: The fit was performed while the rise time constant $\tau_{2}$ was fixed at various values between 10 and $100 \mathrm{fs}$. An exemplary dataset and fit results for various $\tau_{2}$ are shown in Fig. 10. As the $\tau_{2}$ is increased above $60 \mathrm{fs}$, a secondary shoulder at $250 \mathrm{fs}$ pump-probe delay develops that, even visually, deviates from the data. To quantify the quality of the fit, the variable $\chi^{2}$, which follows the total deviation between data and fit, is displayed in the inset. The small value of $\chi^{2}$ between $\tau_{2}=10$ and 60 fs indicates optimal fitting in this range. The significant increase of $\chi^{2}$ above $\tau_{2}=60 \mathrm{fs}$ quantifies the decrease in fit quality already observed visually as the shoulder appearing in the fit results. It is, therefore, concluded that the rise time constant $\tau_{2}$ of the employed empirical mode cannot be larger than $60 \mathrm{fs}$. A clear global optimum is not observed, as the fitting is comparably good for all $\tau_{2}<60 \mathrm{fs}$. This means that in this simplified model, a rise of intensity, interconnected to the creation of DOS, has to happen with a time constant of $60 \mathrm{fs}$ or faster. The phase transition to the transient metallic phase happens, thus, on a timescale on the order of or faster than the time resolution of the experiment.

A bottleneck behaviour [46] is not observed within the temporal resolution of this experiment. The transition from monoclinic insulating $\mathrm{VO}_{2}$ to a highly excited transient metallic state occurs quasi-instantaneously with the exciting laser pulse and is most likely not mediated by the crystal lattice. The observation of such a fast band gap collapse is a very strong indication for an electronically driven process. If a finite rise time of the DOS build-up in the gap exists, it could, on the one hand, result from the screening timescale of quasi-free, photoexcited carriers in $\mathrm{VO}_{2}$ or, on the other hand, also be entirely explained by a response that is delayed with respect to the driving laser pulse due to a 
coherence/polarisation build-up described correctly only in the framework of optical Bloch equations. ${ }^{11}$ This delay would be an effect of electronic population only [87, 88]. Both of electronic origin, these processes strongly suggest an electronic mechanism for the IMT. This finding of a quasi-instantaneous band gap collapse does not only strongly imply an IMT in $\mathrm{VO}_{2}$ before significant ionic motion has occured. It also opens up new directions for the theoretical treatment of the electronic phase transition, as the experimental results show that it occurs when the ions are still in their monoclinic positions and a frozen lattice approach can be used.

Band structure calculation of photoexcited monoclinic $\mathrm{VO}_{2}$. Theoretical investigations of $\mathrm{VO}_{2}$ and its phase transition(s) were only recently able to reproduce a band gap in the equilibrium low-temperature monoclinic phase $[21,27,29]$. Rubio's group ${ }^{12}$ calculated the monoclinic ground state used as a starting point for photoexcitation by a first-principles Greens functions approach (the self energy $\Sigma$ is calculated in the $G W$ approximation, where $G$ is the single particle Green's function and $W$ the screened Coulomb potential) in combination with DFT calculations [5]. ${ }^{13}$ The resulting electronic band structure of monoclinic $\mathrm{VO}_{2}$ exhibits an indirect band gap of $0.65 \mathrm{eV}$, close the experimental value of $0.6 \mathrm{eV}$ [33] (not shown). It is important to note that when self-consistency is obtained on the COHSEX level as done here, screening is considered in a static approximation, leading to the correct opening of a band gap in these calculations.

Fig. 11a) shows the calculated DOS of monoclinic $\mathrm{VO}_{2}$ (blue) ${ }^{14}$ In order to "simulate" the experimental situation after photoexcitation and to calculate the electronic structure of the transient phase, electrons from the valence band were transfered to the conduction band of the ground state, i.e. redistributed leading to an occupation of different orbitals. The band structure is then recalculated in the framework of the $G W$ approximation. The photoexcitation is mimicked by modifications of the occupations similar to the experimental conditions, using an "excitation density" of $7.5 \%$ of the $3 d$ electrons in the vanadium atoms [5,82]. In the calculation, the occupation numbers are reduced for occupied and increased for unoccupied states by the same amount (i.e. generating an equal amount of electrons and holes). This occupation change affects the screened Coulomb interaction $W$, and, thus, the band structure is recalculated using this modified $W$ due to the presence of quasi-free carriers. The resulting DOS of the excited state (red) exhibits an extraordinarily large band gap renormalization; both, valence and conduction band shift by almost half an $\mathrm{eV}$ towards each other and, thereby, lead to a collapse of the band gap. While band gap renormalizations due to free-carrier doping are well-known also in "ordinary" semiconductors [52, 89-93], it usually leads to a moderate narrowing of the gap (which can be enhanced if structural modification is involved, see for instance [52]) and never resulted in a complete collapse. Fig. 11b) compares the experimental and theoretical results. To do so, the calculated DOS is multiplied by a Fermi-Dirac distribution function at $T=300 \mathrm{~K}$. The qualitative agreement of measured data and calculations is very good. Deviations can be explained by the fact that the experiment might very well not integrate over all k-space, in contrast to the calculated DOS. ${ }^{15}$

To gain additional insights into the microscopic origin of the band gap collapse, different occupation distributions (with the same number of total electron-hole-pairs) are used and the results are compared in Ref. [5]. In particular, Rubio et al. calculated the electronic band structure of monoclinic $\mathrm{VO}_{2}$ when only doping holes to the top of the valence band, neglecting electron doping to the conduction band. ${ }^{16}$ The resulting DOS is depicted in Fig. 11a) (shaded area), exposing a weaker renormalization, but persistent band gap collapse. It should be noted that pure electron doping also leads to a renormalization of the band gap, however, by far not as strong as hole doping and insufficient to make $\mathrm{VO}_{2}$ metallic at these excitation densities (see [5]). This shows that the creation of holes in the localised states at the top of the VB (corresponding to the bonding orbitals of the V-dimers along the monoclinic c-axis) is the main driving mechanism leading to the dramatic band gap renormalization, as suggested previously

\footnotetext{
${ }^{11}$ Optical Bloch equations were omitted when modelling the temporal response, because the dephasing times are not known.

${ }^{12}$ The presented results have been obtained in close collaboration with Angel Rubio, Lede Xian, Pierluigi Cudazzo (Universidad del País Vasco, San Sébastian ), and Matteo Gatti (École Polytechnique, Palaiseau). See Ref. [5] for details.

${ }^{13}$ The monoclinic crystal structure was calculated using DFT+LDA. After that, 27 iterations of self-consistent COHSEX (a static approximation to $\mathrm{GW}$ ) plus 9 iterations of perturbative $G_{0} W_{0}$ calculations are used $[28,29]$. Here, the previously calculated eigenvalues and orbitals are recycled and self-energy corrections are evaluated.

${ }^{14}$ The monoclinic gap size of $0.77 \mathrm{eV}$ slightly overestimates the experimental value, because the final $G_{0} W_{0}$-step has been omitted to reduce computational cost. Nevertheless, qualitatively, the results remain valid.

${ }^{15}$ Without scattering processes, only low momenta around the $\Gamma$-point are probed with the low photon energies that were used in the experiment.

${ }^{16}$ Charge neutrality is preserved by a homogeneous, negatively charged background
} 


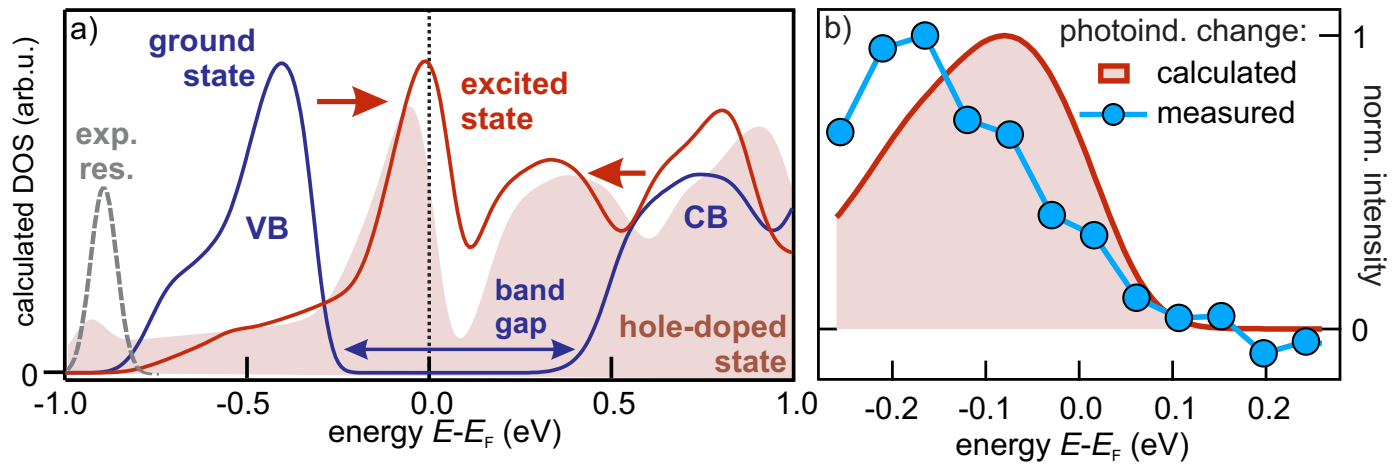

Figure 11: a) Integrated DOS for monoclinic phase before (blue) and after (red) redistribution of 0.3 electrons per unit cell from the valence to the conduction band, broadened by the experimental resolution of $85 \mathrm{meV}$ (grey dashed Gaussian). Clearly, the band gap collapses by a strong renormalization of VB and $\mathrm{CB}$. The shaded area shows the calculated DOS when only hole doping is applied, which is also sufficient to render $\mathrm{VO}_{2}$ metallic. Modified from [5]. b) Comparison of the experimental PE spectrum of the transient phase (taken from Fig. 8) with the theoretical excited state from a), assuming a Fermi distribution at $300 \mathrm{~K}$. Modified from [82].
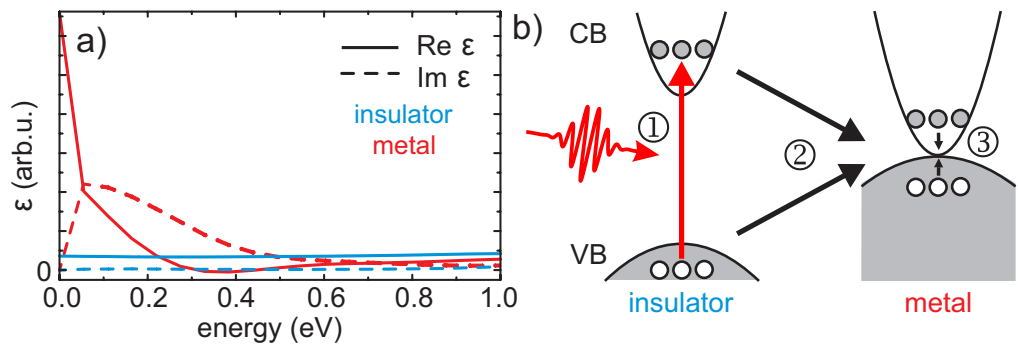

Figure 12: a) Real and imaginary part of the dielectric function of $\mathrm{VO}_{2}$ for the monoclinic phase before (blue) and after (red) redistribution of 0.3 electrons per unit cell from the valence to the conduction band. Adopted from [5]. b) Elementary steps of the photoinduced electronic phase transition: (1) photoexcitation promotes electrons to the $\mathrm{CB}$ and leaves holes in the VB. (2) Intraband transitions increase the screening of the CIA and thereby close the band gap. (3) Thermalisation of carriers within the energetic region of the former gap occurs slower than this band gap collapse. Modified from [5].

[94]. This conclusion provides insight into the microscopic origin of the phase transition: The calculated dielectric functions in Fig. 12a), for initial insulating (blue) and transient metallic electronic structure (red), exhibit a large change at low energies. It results from the significant change of screening of the CIA that is caused by the creation of holes at the top VB. As these states have a strongly localised character, their depopulation opens up a large amount of new phase space for low-energy excitations, as intraband transitions are suddenly possible.

\subsection{Summary: Instantaneous band gap collapse in photoexcited $\mathrm{VO}_{2}$}

Fig. 12b) gives an overview of the results from the time-resolved PE experiments and the many body pertubation theory calculations. The initial step, the photoexcitation, promotes electrons (grey circles) from the VB to the CB (1). Both, excited electrons and holes, provide the possibility of new intraband transitions, however - due to the higher joint density of states in the top VB states that are originating from the V-V dimer orbitals [5, 29] - the low-energy excitations caused by the photoholes dominate the subsequent band gap renormalization. The change of the screening of the CIA through the free carriers sets the timescale for the band gap collapse (2), which occurs, according to the experiment, faster than $60 \mathrm{fs}$. This is in good agreement with the timescales estimated from the plasmon energy of the equilibrium metallic phase discussed in section 1.1 and with plasma screening timescales in the literature [42-44]. From the calculated zero crossing of the real part of the dielectric function of photoexcited $\mathrm{VO}_{2}$ (cf. Fig. 12a)), the 
plasmon energy of the transient phase can be estimated to be $0.45 \mathrm{eV}$, corresponding to 9.2 fs. ${ }^{17}$. Thus, screening can be considered "instantaneous" on the timescale of the presented experiment. The carrier thermalization dynamics occur on even slower timescales ((3), $200 \mathrm{fs})$.

These results show that the photoinduced electronic phase transition occurs instantaneously with the photoexcitation, i.e. before significant ionic motion or carrier relaxation takes place. In particular, this means that a structural bottleneck of $80 \mathrm{fs}$, as proposed earlier [46] does not hinder the IMT. The observed minimum timescale in the dielectric response, however, may well have its origin in structural dynamics, which are known to occur on multiple timescales and may affect photoinduced changes to the optical response differently at different frequencies. The step-like response observed in the THz conductivity (90-100 meV) by Leitenstorfer and coworkers (Fig. 3 b), c) and $[8,49,50])$, on the contrary, agrees well with the observation of an instantaneous gap collapse and major change of the dielectric function at low energies. The instantaneous band gap collapse, which goes along with the generation of free carriers and which is a consequence of the enhanced screening of the transient phase, however, needs to be discussed in relation to the crystallographic changes induced by photoexcitation that are subject of the following chapter.

\section{The potential energy landscape of $\mathrm{VO}_{2}$ and optical properties during the PIPT}

Here, we focus on the ultrafast changes to the lattice potential induced by strong photoexcitation of monoclinic $\mathrm{VO}_{2}$ and the resulting optical response. We begin with a characterization of the optical properties during the thermal transition (section 4.1) and investigate the two equilibrium phases after weak pertubation (i.e. without driving the PIPT) using time-resolved broadband optical spectroscopy and find coherent phonon oscillations in the monoclinic phase, which vanish in the metallic rutile phase (section 4.2). Their amplitude can be used as an order parameter for the transition of the lattice potential. We then show that, similar to the IMT dynamics discussed in the previous chapter, strong perturbation abruptly drives the system out of the monoclinic into a transient phase (section 4.3). Due to the structural sensitivity of coherent phonon spectroscopy, we are able to show that the transient phase is not only a metal with a monoclinic ion arrangement, but that it has already lost the monoclinic lattice potential symmetry during the timescales of photoexcitation. Furthermore, it is demonstrated that the time-dependent optical response of the transient phase, despite being metallic, does not resemble the one of the equilibrium, rutile phase before 200 fs have ellapsed, the timescale of carrier relaxation (section 3.3) and V-dimer melting [6].

\subsection{Optical properties during the thermal transition}

WLC pulses are used to monitor the temperature-dependent changes in reflectivity over the whole visible wavelength range, i.e. significantly larger than the band gap energy. The heating arm of a hysteresis curve is shown in Fig. 13 a) for probe wavelengths between 500 and $700 \mathrm{~nm}$ (bottom axis). The reflectivity change is normalised to the reflectivity at $300 \mathrm{~K}$. The left axis displays the sample temperature. Before crossing the transition temperature $\left(T_{\mathrm{C}}\right)$, the reflectivity increases slightly with temperature at wavelengths below $600 \mathrm{~nm}$, while - above $600 \mathrm{~nm}-$ it stays constant. When crossing $T_{\mathrm{C}}$, the reflectivity drops at all probed wavelengths as illustrated by the lineouts in Fig. 13b), which are normalised to the signal at $335 \mathrm{~K}$ to highlight changes in reflectivity initiated by the phase transition. Fig. 13c) shows the reflectivity change upon crossing $T_{\mathrm{C}}$. The comparison of two horizontal line-outs above (red) and below (blue) the transition temperature demonstrates that the thermally induced change to the reflectivity is strongest for long wavelengths $(63 \%$ at $700 \mathrm{~nm})$ and decreases linearly with decreasing wavelength $(<10 \%$ at $580 \mathrm{~nm}$ ). This behaviour can be rationalized by the $\mathrm{VO}_{2}$ band structure changes during the transition (Fig. 13d)): Long wavelengths probe transitions between the $\mathrm{V} 3 \mathrm{~d}_{\|}$bands, which change strongly during the phase transition. The shorter the wavelength, however, the stronger the influence of transitions from $\mathrm{O} 2 \mathrm{p}$ to V $3 \mathrm{~d}$ states, which are less affected by the change in band-structure in the vicinity of the Fermi energy [96].

\subsection{Transient optical response in the low-and high-temperature phase after weak excitation}

We use laser pulses centred at $800 \mathrm{~nm}$ to excite insulating $\mathrm{VO}_{2}$ above its band gap. An exemplary trace of the transient change of reflectivity at the same wavelength, measured at room temperature, is shown in Fig. 14a) for a pump fluence of $1.7 \mathrm{~mJ} \mathrm{~cm}^{-2}$, i.e. well below the PIPT threshold. A sudden drop of reflectivity, followed by a slow recovery, is superimposed by beating phonon oscillations.

\footnotetext{
${ }^{17}$ Calculated for smallest momentum transfer in only one direction. For a charge redistribution of $10 \%$ per $\mathrm{V}$ atom and momentum transfer in three directions, plasmon energies between 0.5 and $0.7 \mathrm{eV}$ can be extracted, depending on momentum transfer [95].
} 


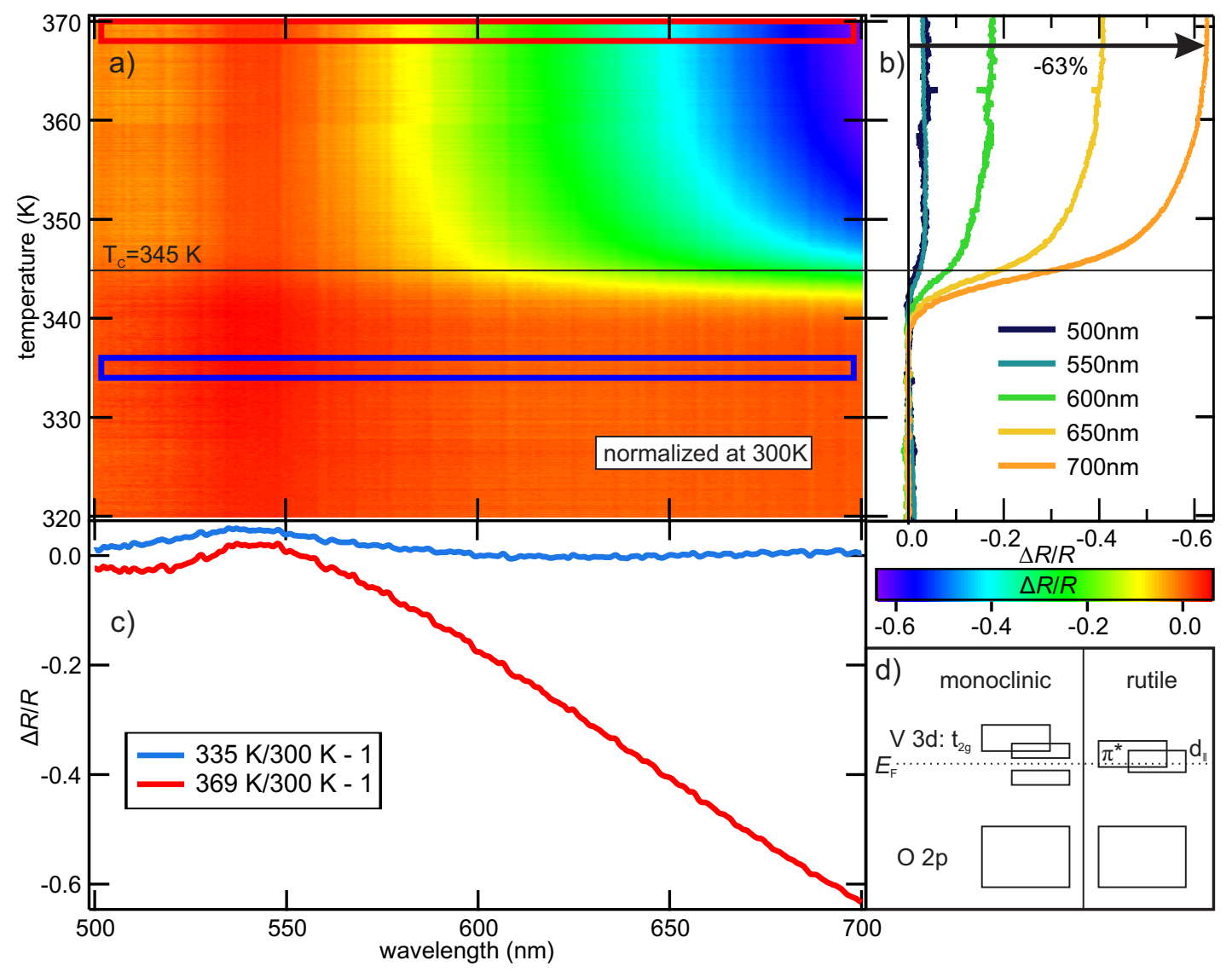

Figure 13: a) Temperature-dependent change of reflectivity in $\mathrm{VO}_{2}$ probed using a WLC between 500 and $700 \mathrm{~nm}$, normalised to measured reflectivity of the insulating phase at $300 \mathrm{~K}$. b) Relative change in reflectivity for selected wavelengths normalised directly below the critical temperature, to only include changes connected to the phase transition. c) Lineouts at indicated positions in a) (red and blue box). d) Sketch of $\mathrm{VO}_{2}$ band structure change. Modified from [7].
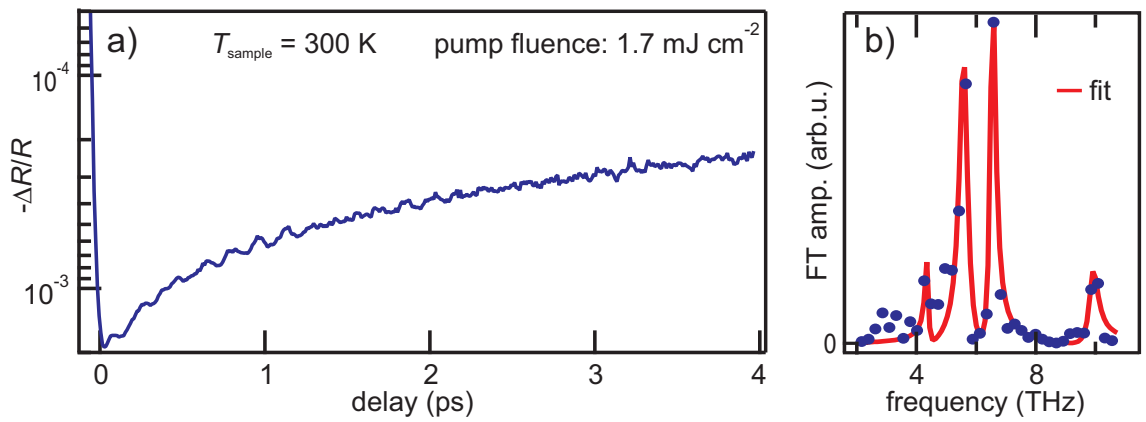

Figure 14: a) Degenerate pump-probe reflectivity transient of $\mathrm{VO}_{2}$ at room temperature upon weak excitation with $800 \mathrm{~nm}$ laser pulses. b) Subtraction of the incoherent background and Fourier transformation yields the coherent phonon spectrum. Modified from [83]. 


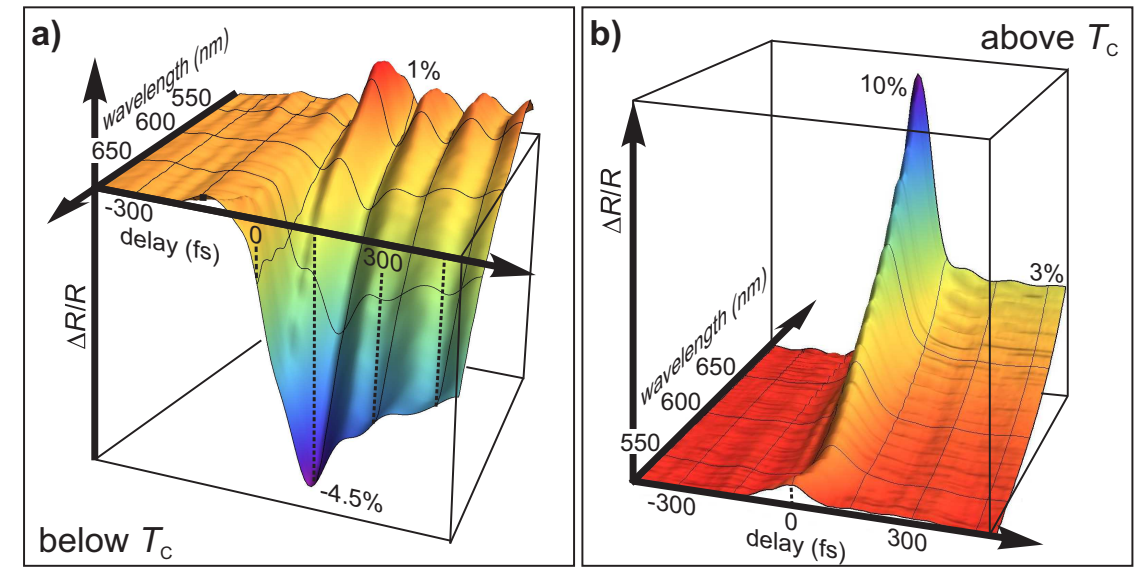

Figure 15: a) Broadband reflectivity change upon pump excitation at room temperature and below phase transition threshold fluence. b) Analogous measurement of metallic $\mathrm{VO}_{2}$ at $\mathrm{T}=400 \mathrm{~K}$. Mind the opposite direction of the wavelength axes in a) and b). Modified from Ref. [83].

To analyse the coherent phonon oscillation, the incoherent (resulting from, e.g., electronic excitation, thermal effects or incoherent lattice dynamics) part is subtracted. It is determined by an empirical fit of the form

$$
\Delta R(t) / R=\left(\operatorname{erf}\left(t / \tau_{\mathrm{p}}\right)+1\right) \times\left(A_{1} \exp \left(-t / \tau_{1}\right)+A_{2} \exp \left(-t / \tau_{2}\right)\right) .
$$

The rising edge, with a width $\left(\tau_{\mathrm{p}}\right)$ that is determined by the pump pulse duration, was modelled by an error function $\operatorname{erf}\left(t / \tau_{\mathrm{p}}\right) . A_{1}$ and $A_{2}$ are the two amplitudes and $\tau_{1}$ and $\tau_{2}$ the decay time constants of the initial recovery of the reflectivity change. Fourier transform (FT) of the remaining coherent part yields the frequency spectrum shown in Fig. 14b). A fit with four Lorentzian line-shapes results in phonon modes at 4.4, 5.7, 6.7, and 10.2 THz. These eigenfrequencies agree very well with the four strongest Raman active phonon modes of the insulating phase previously measured by Raman spectroscopy $[13,97,98] .{ }^{18}$

Probing the transient dynamics with broadband WLC pulses instead of $800 \mathrm{~nm}$ light extends the amount of retrievable information. Fig. 15a) shows a transient trace with respect to pump-probe delay and probe wavelength. Again, a strong incoherent change of reflectivity after photoexcitation is superimposed by coherent phonon oscillations. The strongest incoherent contribution is observed at long wavelengths, as these are closest to the pumping wavelength of $800 \mathrm{~nm}$ where the dielectric function is initially perturbed. Remarkably, the incoherent contribution is close to zero for wavelengths further away from the pump wavelength (i.e. at higher photon energies). Here, only one coherent phonon mode (at $5.7 \mathrm{THz}$ ) dominates the spectrum as shown by a detailed analysis of the phonon spectrum over the whole probing range in Ref. [82]. This qualifies especially this wavelength region for studies of the phonon as a marker of the monoclinic phase symmetry during the PIPT of $\mathrm{VO}_{2}$ upon strong excitation. However, before focusing on the transient phase in section 4.3, we discuss the differences between the transient optical response of the equilibrium, high- and low-temperature phase of $\mathrm{VO}_{2}$.

The transient reflectivity change of $\mathrm{VO}_{2}$ at $400 \mathrm{~K}$ probed by WLC pulses is shown in Fig. 15b). The sign of the reflectivity change is positive at all wavelengths and the overall shape of the transient is very similar to the response probed at $800 \mathrm{~nm}$ (not shown, see Ref. [82]). The peak amplitude as well as the transient background are strongest at long wavelengths and decrease towards shorter wavelengths. Again, the dielectric function is affected most strongly close to the pump wavelength. Clearly, no coherent phonons are observed in the case of metallic $\mathrm{VO}_{2}$, which can be substantiated by a detailed analysis of the Fourier transform (FT) of Fig. 15b) over the whole probing range (not shown, see Ref. [82]). The coherent phonon amplitudes of the low-temperature phase can, therefore, be used as a marker for the monoclinic phase and as an order parameter for the phase transition of the lattice potential symmetry in $\mathrm{VO}_{2}$.

\footnotetext{
${ }^{18}$ Other strong modes with higher frequencies cannot be coherently excited/resolved because of the pulse duration of pump and probe laser pulses on the order of $40 \mathrm{fs}$.
} 


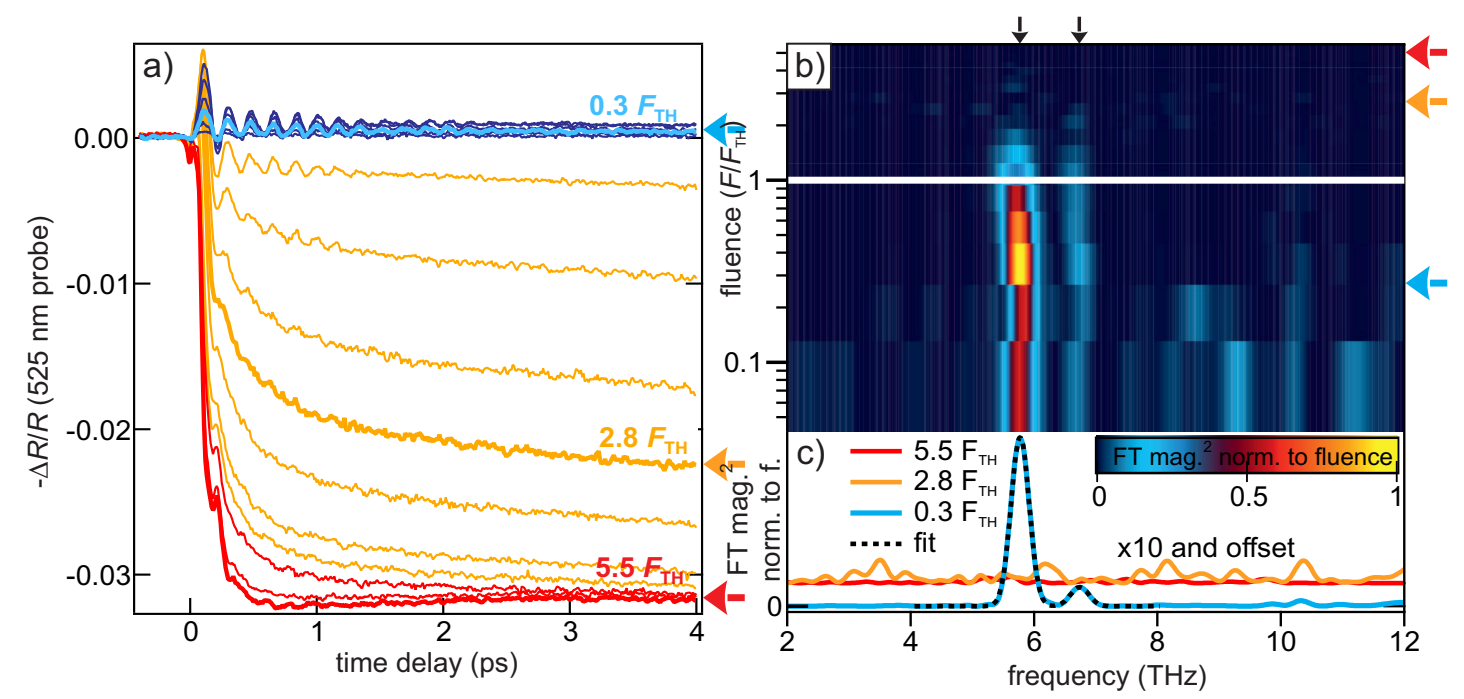

Figure 16: a) Photoinduced reflectivity changes probed by the $525 \mathrm{~nm}$ part of the WLC pulses for various excitation fluences $\left(\lambda_{\text {pump }}=800 \mathrm{~nm}\right)$ below (blue) and above (orange) the phase transition threshold $F_{\mathrm{TH}}$ and in the saturation regime (red). To achieve a superior signal to noise ratio as compared to broadband measurements employing an optical spectrometer, lock-in detection was used after filtering the reflected WLC probe at $525 \mathrm{~nm}$ (bandwidth: $5 \mathrm{~nm}$ ), therefore keeping the temporal shape of the WLC pulse intact at the time of probing. b) False colour plot of Fourier transforms of the transients in a) after subtraction of the incoherent background as discussed in section 4.2 and as a function of normalised fluence. Intensities are normalised to the respective pump fluence. c) Fourier transforms for three distinct transients representing the three regimes: below (blue) and above (orange) threshold and in saturation (red). The respective positions are indicated by the coloured arrows in a) and b). Fitting with two Gaussian peaks yields the dotted black curve in c). Adopted from [82].

The spike-like feature in the optical response of the rutile metallic phase of $\mathrm{VO}_{2}$ has a decay constant on the order of 20 fs (not shown, see [82]) and could either result from a critically damped phonon oscillation of the rutile phase or in electronic excitations in the corresponding metallic band structure. As the carrier relaxation time in photoinduced monoclinic metallic (section 3.3) and in photoexcited rutile metallic $\mathrm{VO}_{2}$ [82] are both on the order of $200 \mathrm{fs}$, we conclude that the spike cannot be related to carrier relaxation dynamics and is probably related to free carrier screening in the photoexcited high-temperature phase. For instance, the dynamics could result from an overdamped (through the free carrier screening) oscillation of a phonon mode of rutile $\mathrm{VO}_{2}$ [13]. Independent of the exact origin, the distinct shape and sign of reflectivity change will be used to track the evolution towards the equilibrium metallic phase response after the photoinduced phase transition in section 4.4.

\subsection{The PIPT threshold}

After describing the ultrafast optical response of the two equilibrium phases of $\mathrm{VO}_{2}$ to weak excitation, the photoinduced phase transition is investigated by gradually increasing the pump-power while staying at room temperature. Here, we focus on the wavelength region around $525 \mathrm{~nm}$, which qualifies for a systematic analysis of the PIPT based on coherent phonons, as the incoherent 'background' part of the transient, seen at longer wavelengths, is comparably low in intensity, while the phonon mode centred at $5.7 \mathrm{THz}$ dominates the phonon spectrum as discussed in the previous section.

The pump-induced transients $\left(\lambda_{\text {pump }}=800 \mathrm{~nm}\right)$ for various excitation densities are shown in Fig. 16a). First, similar to previous studies (cf. e.g. Refs. $[6,8,48,57]$ ), the phase transition threshold has been determined at the point where the transient response at 1 ps time delay starts to be non-linear with fluence (cf. Fig. 17a), green, $F_{\mathrm{TH}}=$ $4.9 \mathrm{~mJ} \mathrm{~cm}^{-2}$ ). Note that, as shown in chapter 3, at this time delay and for above threshold excitation, $\mathrm{VO}_{2}$ is already metallic, the carriers have relaxed and the electronic structure remains unchanged for hundreds of ps. Each transient was integrated over a window of $190 \mathrm{fs}$, corresponding to one phonon period, to average out reflectivity changes due to the phonon oscillation. Based on this analysis, the transients in Fig. 16a) are colour-coded and clearly show that, below threshold (blue curves), coherent phonon oscillations dominate without any significant background intensity. Above 
threshold (orange), the coherent phonon signatures start vanishing and a long-lived transient background increases with fluence and eventually saturates.

Using the nonlinearity of the reflectivity change at 1 ps time delay (Fig. 17a), green) is of course an arbitrary choice. Moreover, it does not carry any direct information about the metallicity of the photoexcited $\mathrm{VO}_{2}$, as reflectivity changes are not exclusively determined by changes of the electronic band structure and even if they would be, they do not necessarily probe a Fermi surface. However, the transient reflectivity measurements do, indirectly, contain structural information through their sensitivity to the monoclinic phase phonon modes, which can serve as an order parameter for the phase transition of the crystal potential symmetry in $\mathrm{VO}_{2}$ as shown in the following.

The Fourier transforms (magnitude squared) of the transients in Fig. 16a) are shown in b) for increasing pump fluences. They are normalised to pump fluence to remove any signatures that depend linearly on excitation density. The threshold determined above is indicated by the white line. Two distinct peaks are observed in the phonon spectrum below $F_{\mathrm{TH}}$, corresponding to the 5.7 and $6.7 \mathrm{THz}$ modes of the insulating, monoclinic phase of $\mathrm{VO}_{2}$. When the fluence is increased above threshold, these modes vanish quickly. To illustrate this further, Fig. $16 \mathrm{c}$ ) shows three phonon spectra, representative of the three regimes (blue: below threshold, orange: above threshold, red: in saturation). The orange and red curves have been scaled up by a factor of ten and offset for clarity. A fit (two Gaussian peaks) to the phonon spectrum of the transient pumped below threshold yields a strong mode at $5.7 \mathrm{THz}$ and a weaker one at $6.7 \mathrm{THz}$, in good agreement with the data shown in Fig. 14b). ${ }^{19}$ Also, a slight softening of the $5.7 \mathrm{THz}$ mode is observed before it disappears, which will be quantified further below.

A first quantification of the $5.7 \mathrm{THz}$ phonon mode amplitude is achieved by finding the position of the first trough and the following maximum of the phonon oscillation in a below-threshold transient, as illustrated in Fig. 17b) and extracting the difference $2 A_{5.7 \mathrm{THz}}$ between both of these two points. The fluence dependence of $2 A_{5.7 \mathrm{THz}}$ is shown in Fig. 17a) and compared to the transient change in reflectivity probed with $525 \mathrm{~nm}$ at 1 ps on a logarithmic fluence axis. The phonon amplitude starts to deviate from the expected linear fluence dependence (indicated by the solid line) at the phase transition threshold $F_{\mathrm{TH}}$, following the same trend as the transient reflectivity change and, thus, similarily qualifying as an order parameter for the PIPT.

The gradual decrease of FT intensity close to threshold excitation is due to inhomogeneous probing of a distribution of differently strongly pumped regions because of the absorption profile normal to the surface. Concurrently with the saturation behaviour of the transients in Fig. 16a), the FTs in panel b) approach zero intensity, indicating homogeneous above threshold pumping of the whole probed area in the high fluence limit. This saturation regime is entered at around $4-5 \times F_{\mathrm{TH}} \simeq 20-25 \mathrm{~mJ} \mathrm{~cm}^{-2}$ [82]. In comparison with the thresholds determined by UED of $4.8 \times F_{\mathrm{TH}}=F_{\mathrm{sat}} \simeq 43 \mathrm{~mJ} \mathrm{~cm}^{-2}$ [6], this saturation fluence is significantly smaller. However, the usual uncertaincies in fluence determination (spot size, absorption/reflection losses due different angles of incidence etc.) can - in combination with different probing volumes of different techniques - easily account for this factor of two. The $\operatorname{similar} F_{\mathrm{TH}} / F_{\text {sat }}$ ratio, on the contrary, strongly suggests that the fluence threshold observed in the optical experiments directly relates to the UED threshold that defines the onset of non-thermal melting of the V-V dimerization within 300 fs [6].

Detailed fitting of the transients before, during, and after crossing $F_{T H}$. In order to quantify the dynamics observed in the optical data close to the photoinduced phase transition, a complex fitting model, including the transient background due to electronic excitation and heating, is necessary ${ }^{20}$. Before, during, and after crossing $F_{\mathrm{TH}}$ different contributions dominate the dynamics as discussed in the following: As mentioned in section 1.2, if the force exerted on the lattice is small and the phase transition is not driven (below threshold), the lattice dynamics (i.e. coherent phonon oscillations) will modulate the total reflectivity $R$ with a contribution according to the monoclinic phases normal modes:

$$
\Delta R_{\mathrm{Q}}(\lambda, t)=\sum_{i} A_{i}(\lambda) q_{i}(t)
$$

with the wavelength-dependent amplitude $A_{i}(\lambda)=\frac{\partial R(\lambda)}{\partial q_{i}}$. The time dependence of the phonon oscillation is therefore independent of wavelength, while its contribution to the reflectivity change is not. The phonon displacement $q_{i}(t)$ of

\footnotetext{
${ }^{19} \mathrm{~A}$ small, residual peak of the $10.2 \mathrm{THz}$ mode is visible in the data, while the $4.4 \mathrm{THz}$ mode is not observed. This is due to the wavelengthdependent probing cross section in addition to their lower relative intensity observed in Raman spectroscopy.

${ }^{20}$ The fitting model has been developed by Simon Wall at the Fritz Haber Institute, Berlin [7].
} 

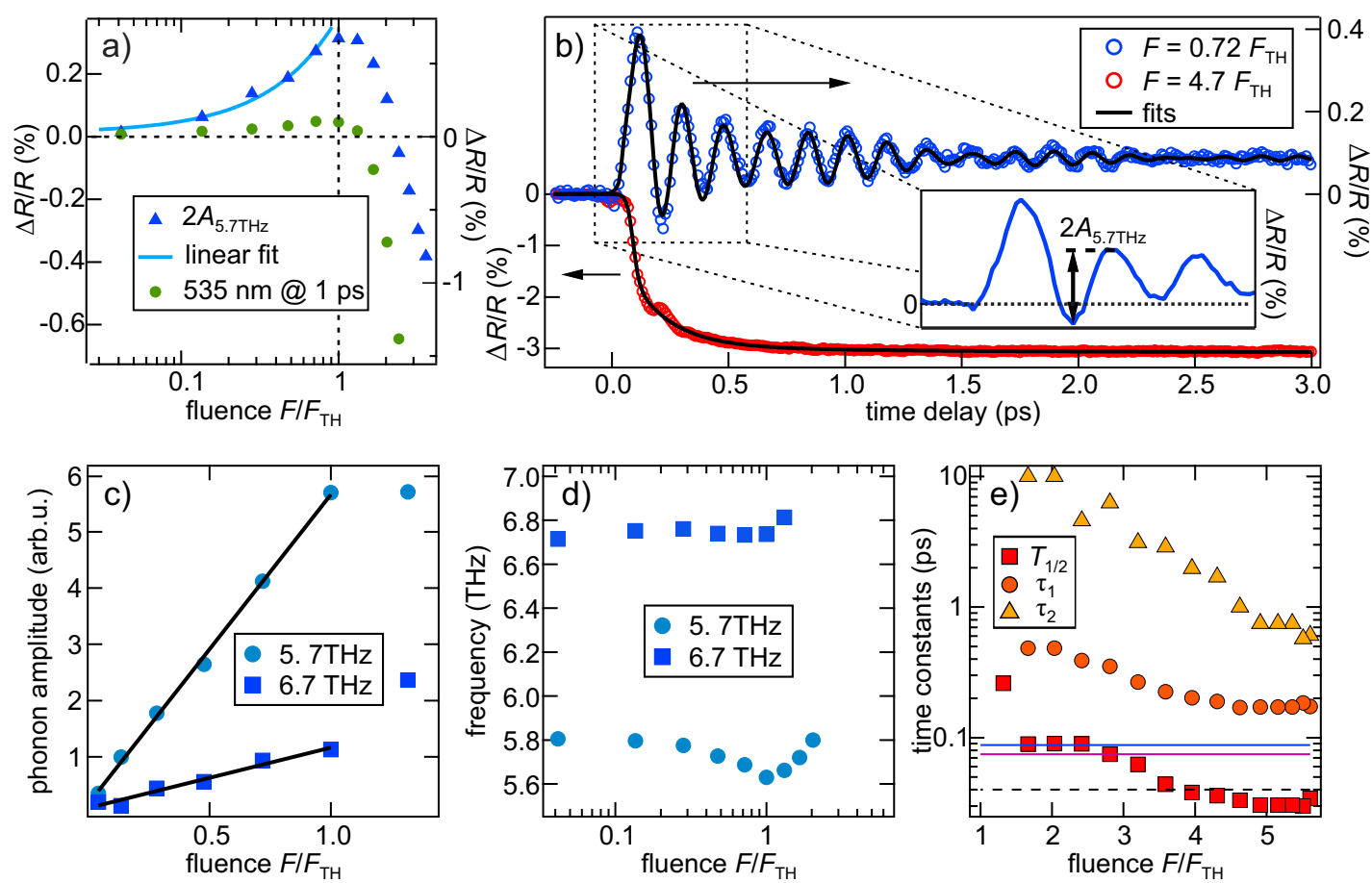

Figure 17: a) Fluence dependence of the double amplitude $2 \times A_{5.7 \mathrm{THz}}$ in comparison to the transient's amplitude at 1 ps averaged over a time window of $190 \mathrm{fs}$ to suppress reflectivity changes due to phonon oscillations. The linear fit does not appear as a straight line because of the logarithmic fluence axis. b) Below- (blue) and above-threshold transient (red) including fits as described in the text. The inset presents a detailed view of the below-threshold transient to illustrate the double-amplitude determination of the $5.7 \mathrm{THz}$ phonon. c) Fluence dependence of phonon amplitude and d) frequency determined by the fit routine. e) Time constants $\tau_{1}$ and $\tau_{2}$ as well as the half rise position of the sigmoid function $T_{1 / 2}$ (orange squares). The solid lines correspond to the half-periods of the 5.7 and $6.7 \mathrm{THz}$ phonon modes and the dashed line to the estimated laser pulse duration. Modified from [7, 82]. 
the normal modes follows a forced, damped oscillation and is determined by damping constant $\xi_{i}$, eigenfrequency $\omega_{i}$ and generating force $F(t)$. The below-threshold transients probed at $525 \mathrm{~nm}$ in Fig. 16a) provide valuable information about the character of this force, whether it is impulsive, step-like or has some intermediate shape (i.e. a finite time constant $\tau_{F}$ ): An impulsive (or quickly decaying) force on the lattice generates a sine-like oscillation around the atomic equilibrium position, while a persistent (displacive) force results in cosine-like oscillations around a new (displaced) position. As there is neither a significant intensity offset (on the order of the phonon amplitude) nor an exponential decay in the equilibrium position around which the phonon oscillates, the data suggests a rather fast decay time constant for the force (i.e. a more impulsive than step-like behaviour). The best fit for all fluences results in a quasi-impulsive force with an exponential decay time of $\tau_{F}=50 \mathrm{fs}$.

In addition to the coherent lattice response, carrier dynamics, heating effects, and other incoherent processes lead to incoherent transient reflectivity changes, which are especially prominent when probing with $800 \mathrm{~nm}$ [7]. When probing with $525 \mathrm{~nm}$, only a constant offset is needed to describe the incoherent part of the transients:

$$
\Delta R_{\mathrm{K}}(\lambda, t)=\Theta(t) K(\lambda)
$$

The rising edge of the pump-induced change is modelled by the sigmoid function $\Theta(t)=\left(1+\exp \left[-\left(t-T_{1 / 2}\right) / w\right]\right)^{-1}$, which has a width $w$ corresponding to the pump-pulse duration and a delayed-rise parameter $T_{1 / 2}$.

Above the phase transition threshold, the phonon modes are lost or become overdamped. Therefore, the previous description of the lattice response has to be modified, because eigenfrequency and damping ratio loose their meaning, when the phonon mode amplitude is zero, or are strongly coupled in the case of an overdamped oscillation. The oscillatory contribution $\Delta R_{\mathrm{Q}}(\lambda, t)$ is then gradually replaced by:

$$
\Delta R_{\mathrm{S}}(\lambda, t)=\Theta(t)\left[\sum_{i=1,2} C_{i} e^{-t / \tau_{C_{i}}}\right] .
$$

The overall change of the reflectivity is simply the sum of the contributions discussed above, but, when increasing the fluence and moving through the 3 regimes (below threshold, above threshold and in saturation), different parts of the fitting model have to be switched off and on manually:

$$
\Delta R(\lambda, t)=\Delta R_{\mathrm{Q}}(\lambda, t)+\Delta R_{\mathrm{K}}(\lambda, t)+\Delta R_{\mathrm{S}}(\lambda, t)
$$

The complete fit is then done by least mean square fitting including numerical solution of the differential equation for the coherent phonon oscillations, when $\Delta R_{\mathrm{Q}}(\lambda, t)$ is included.

A first round of fitting using $\Delta R_{\mathrm{Q}}(\lambda, t)$ and $\Delta R_{\mathrm{K}}(\lambda, t)$ yielded constant results for the electronic decay time constants $\tau_{i}$, which were, therefore, held fixed for a second run of fits at $\tau_{1}=250$ fs and $\tau_{2}=1.42$ ps to convert random errors (due to fluctuations) to systematic errors and yield more consistent results.

Fig. 17b) shows a transient and its fit for below threshold photoexcitation in blue. The model includes the two strongest phonon modes at 5.7 and $6.7 \mathrm{THz}$ and reproduces the data nearly perfectly. The amplitudes of the two phonon oscillations are displayed in Fig. $17 \mathrm{c}$ ) as a function of normalised fluence $F / F_{\mathrm{TH}}$. Both grow linearly with fluence (black lines) up to the threshold fluence, in agreement with the direct extraction of the phonon amplitudes in panel a).

The fluence dependence of the phonon eigenfrequency in Fig. 17d) shows, that the $5.7 \mathrm{THz}$ mode becomes slightly softer towards the photoinduced phase transition; a behaviour, which is not observed when thermally driving the transition [37]. This photoinduced change of the connected force constant, can be assessed as an indication for a stronger coupling of the $5.7 \mathrm{THz}$ mode to the electronic (photo-)excitation than to the thermal driving mechanism. Having $A_{g}$ and $B_{g}$ symmetry (and, thus, being non-polar), none of the coherent phonons modes observed must be renormalized through interaction with the photoexcited plasma as, for instance, in GaAs [42]. As discussed in the introduction, $\mathrm{VO}_{2}$ is not a true Peierls system, where one mode maps one structure onto the other, thus leading to a phonon mode softening close to the transition until it vanishes. The $5.7 \mathrm{THz}$ mode (tiliting of the dimers) can be considered to be a spectator mode of the transition that vanishes above threshold, because the lattice potential symmetry is reduced as shown further below.

The increase of its frequency back to the initial value, when pumping above the threshold fluence could be an artifact, due to inhomogeneously exciting the $\mathrm{VO}_{2}$ thin-film sample: The reflectivity drops in the immediate surface 
region, where pump-light is absorbed most, because the material is driven across the phase transition. Hence, the backside of the film, which is still in the insulating phase, is probed to a larger extent. Therefore, the phonon is probed more prominently at the backside of the film, where its frequency has not gone soft.

For fitting the transition region just above threshold, all components of the fitting model have to be "switched on", as a distribution of regions where the fluence is below and above the threshold fluence is probed and residual oscillations are visible in the transients. When the oscillations clearly vanish, $\Delta R_{\mathrm{Q}}(\lambda, t)$ is manually excluded from the fitting model. An exemplary above threshold transient $\left(F=4.7 F_{\mathrm{TH}}\right)$ in the saturation regime and the corresponding fit are shown in Fig. 17b). The fit reproduces the data nicely, and for $t>300 \mathrm{fs}$ there is certainly no oscillatory component at the phonon eigenfrequencies visible above noise level. However, at earlier delays frequency components are observed that would be compatible with the 5.7 and $6.7 \mathrm{THz}$ modes, which could be explained by various scenarios:

- strongly damped phonon within the (so far) unchanged monoclinic lattice potential

- strongly damped phonon of the photoexcited phase similar to the one postulated in [48]

- temporal feature of the pump-laser pulse that becomes relevant due to the high excitation intensities

- a high order non-linearity, common in broadband pump-probe measurements [99]

At fluences below $F_{\mathrm{TH}}$, the amplitude of the oscillation rises linearly and abruptly drops upon crossing the threshold (Fig. 17a)). Further increase of the excitation density results in the build-up of the above-mentioned oscillatory component at early delays. Its amplitude increases with increasing excitation density and it is out-of-phase with the oscillations observed in the monoclinic phase for low excitation densities (cf. Fig. 16a)) and Fig. 17b)). It, thus, seems improbable that the two oscillations have the same origin. Along similar lines, it will be shown in section 4.4 that, in the transient metallic phase created by above-threshold excitation, it is neither possible to coherently control (i.e. suppress or enhance) the high-fluence oscillatory component at early delays by a second, time-delayed pump pulse nor to excite any other coherent contribution to the transient reflectivity. It seems likely that the high-fluence oscillatory component at early delays results from either a phonon characteristic of the transient phase or from a non-linear effect connected to pump and/or probe pulse. Taking into account the similarity to the oscillation before time zero and the inability to coherently control the signal (cf. section 4.4), the latter seems to be the more likely explanation.

In any case it is very clear already from this analysis that at delays later than $300 \mathrm{fs}$, there is no phonon mode of the monoclinic phase visible anymore. The disappearance of these modes could be caused by a LPC or, simply, by the IMT transition that occurs instaneously with the photoexcitation (chapter 3). In the latter case, the free carriers of the transient phase could either (i) dominate the optical response, making phonon detection difficult as discussed in [100] or (ii) enhance the damping/dephasing of the phonons.

(i) As shown in Fig. 15, the ratio of coherent and incoherent contributions to the transient reflectivity strongly depends on the probe wavelength and is, at least for $\mathrm{VO}_{2}$, most cumbersome when approaching the pump wavelength. In our broadband experiments, the monoclinic modes vanish at all investigated probe wavelengths. Further, the IMT generates only 10-20 times more free carriers than the photoexcitation just below $F_{\mathrm{TH}}$. As the latter do not affect the phonon mode amplitudes below threshold, which increase perfectly linearly below $F_{\mathrm{TH}}$, and as the incoherent background of the transient phase is only a factor of six larger than the maximum oscillation's amplitude (Fig. 16a)), we believe that the pure build-up of free carrier optical response could not fully overlay the phonon signal.

(ii) Above threshold, the incoherent contributions to the change of reflectivity become increasingly dominant as discussed in detail in Ref. [7] and could be interpreted as overdamped phonons of the monoclinic phase. The dependency of time constants $\tau_{1}$ and $\tau_{2}$ (cf. Eq. (5)) of the incoherent dynamics on fluence (above threshold) is shown in Fig. 17e). In the case of an overdamped phonon the time constant should increase with fluence or, for a strictly impulsive excitation force, decrease close to critical damping [82]. Clearly, this is not the case, showing that the two exponential decays do not result from an increasingly overdamped phonon of the monoclinic phase. On the contrary, the final value of time constant $\tau_{1} \sim 1 \mathrm{ps}$ is on the same order of magnitude as the buildup time of several UED peaks, interpreted as a collective reorganization of the valence charge density, not a structural rearrangement [6]. Furthermore, $\tau_{1} \sim 200 \mathrm{fs}$ coincides with the hot carrier thermalisation time of the 
photoinduced-metallic phase measured by time-resolved photoemission (chapter 3), suggesting that it reflects the impact of hot carriers on the dielectric function.

Based on these considerations, we conclude that the vanishing of the monoclinic phonon modes most likely results from a photoinduced change of the lattice potential. This conclusion will be substantiated in the following section by the investigation of the transient response of the photoexcited phases above and below threshold excitation.

A frequently used measure for the timescale of PIPTs is the rise time of the optical response. The fluence dependence of the half-rise position $T_{1 / 2}$ of the sigmoid function (Eqs.(4) and (5)) is displayed in Fig. 17e) (orange squares). Below saturation, it is similar to the half-periods of the 5.7 and $6.7 \mathrm{THz}$ phonon oscillations (i.e. 88 and $74 \mathrm{fs}$, indicated by the solid lines) and, thus, on the same order of magnitude as the 'structural bottleneck' timescale reported in [46] and introduced in section 1.1). Such a bottleneck is not observed here upon crossing $F_{\mathrm{TH}}$, as the half-rise position $T_{1 / 2}$ decreases even below the pump pulse duration (i.e. $<40 \mathrm{fs}$ indicated by the dashed line and labeled $\tau_{\mathrm{P}}$ ) upon increasing the fluence into the saturation regime. This indicates that the non-thermal phase transition out of the equilibrium monoclinic phase can be driven faster than the pump pulses temporal envelope.

From the above analysis, we conclude that the amplitude of a monoclinic phase phonon mode can be used as an order parameter that vanishes (cf. Fig. 16) when the system is driven out of the monoclinic phase crystal potential symmetry. In order to elucidate how fast the monoclinic crystal potential symmetry is lost, the next section uses coherent phonon spectroscopy of the transient phase of strongly photoexcited $\mathrm{VO}_{2}$.

\subsection{Dynamics of the photoexcited state}

In line with the investigations of the transient response in the equilibrium insulating and metallic phases of $\mathrm{VO}_{2}$, presented in section 4.2, the transient response following weak perturbation of the photoexcited state (below threshold) and of the transient phase (above threshold) is investigated in this section. To do so, a three pulse scheme, as depicted in Fig. 18a) is applied: While the established pump-probe (two-pulse) scheme monitors the transient optical response caused by photoexcitation, the pump-pump-probe scheme allows the investigation of the change of the transient optical response as a function of time after the initial photoexcitation. A first laser pulse P1 is used to excite the system (below or above threshold, depending on its fluence) while a second, time-delayed, low-intensity pump pulse $\mathrm{P} 2$ acts as a weak perturbation ${ }^{21}$, triggering dynamics of the photoexcited state. These dynamics are monitored by a probe pulse, with varying time delay with respect to P1 (and P2). This scheme is applied to below and above threshold excitation with P1 to elucidate the nature of the state after photoexcitation. The evaluation focusses on the coherent phonon responses.

When the fluence of P1 is below the threshold, the transient response of the photoexcited insulating phase is probed. Here, as described above, the most prominent dynamic features are modulations of the reflectivity due to oscillations of coherent phonons of the monoclinic symmetry. A coherent phonon, which has been excited by P1 and is less than critically damped, can be coherently controlled by P2. Trivially, it can also be excited again after the first phonon excitation has decayed/dephased. As the coherent phonon oscillation is characteristic for the symmetry of the crystal potential, it can only be excited if this symmetry persists.

For the coherent control experiments, the time delay between P1 and P2 has to be tuned in- and out-of-phase with respect to the $5.7 \mathrm{THz}$ coherent phonon period, which dominates the optical response at a probe wavelength of $525 \mathrm{~nm}$. For this mode, we used a delay between the two exciting pulses P1 and P2 of $\sim 88$ fs for out-of-phase $(\phi=\pi, 3 \pi, \ldots)$ and $\sim 175$ fs for in-phase $(\phi=2 \pi, 4 \pi, \ldots)$ excitation. The transient responses for a total pump fluence below threshold $\left(F_{\mathrm{P} 1}=4.4 \mathrm{~mJ} \mathrm{~cm}{ }^{-2}, F_{\mathrm{P} 2}=1.7 \mathrm{~mJ} \mathrm{~cm}^{-2}, F_{\mathrm{P} 1}+F_{\mathrm{P} 1}<F_{\mathrm{TH}}\right)$ is summarised in Fig. 18b) and c) for different P1-P2 delays: The black curve in b) shows the transient reflectivity change due to P1 alone. The delay axis is plotted with respect to the temporal overlap between probe and P1. The coloured arrows indicate the temporal positions for in- and out-of phase excitation with a second pulse $\mathrm{P} 2$. The transients after double excitation are colour-coded to match the arrows at the top. They show, that for out-of-phase excitation the phonon amplitude is minimised (blue and yellow thick line), while in-phase excitation yields an amplified phonon amplitude (green thick line).

When subtracting the pure P1 response, the transient P2-induced dynamics in the photoexcited state remain, as shown in Fig. 18c). The transients look essentially the same as the one excited by a single pump pulse (e.g. P1 only),

\footnotetext{
${ }^{21}$ The fluence was kept below threshold to ensure that $\mathrm{P} 2$ does not drive the transition.
} 

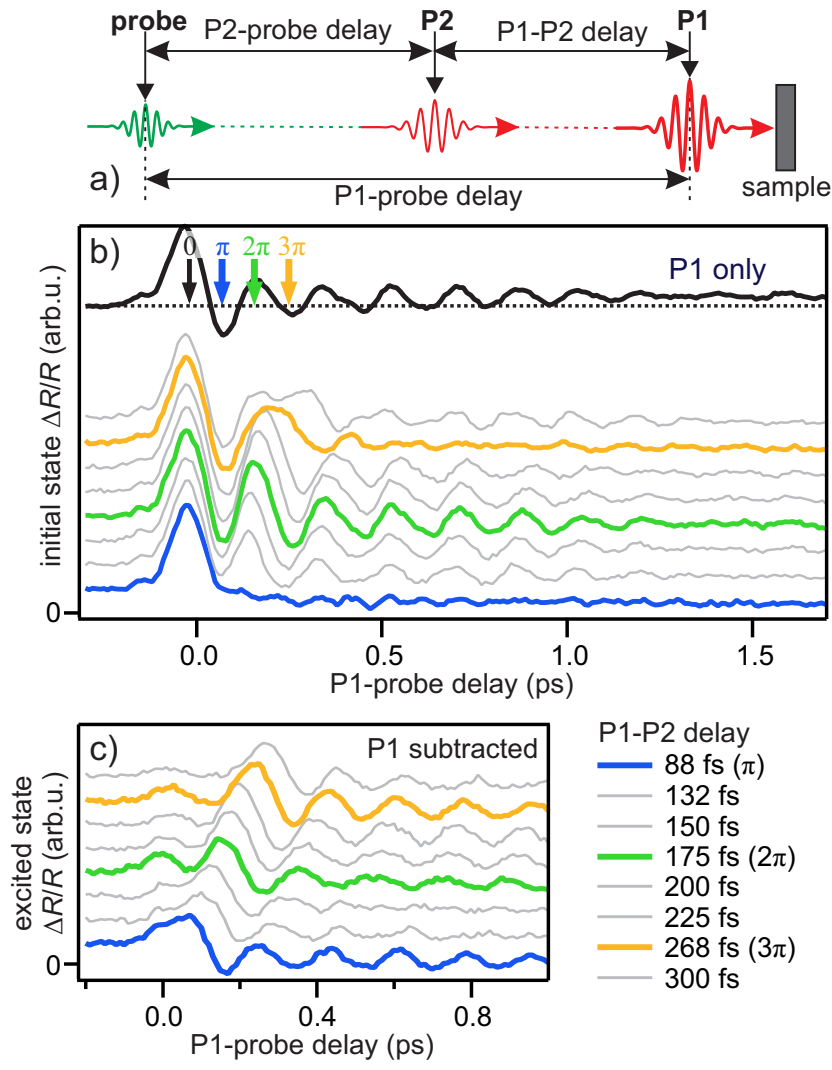

Figure 18: a) Pump-Pump-Probe scheme for the investigation of the dynamics of photoexcited $\mathrm{VO}_{2}$ : First pump pulse P1 (red, bold) drives the system into an excited state, or even into the transient phase (depending on the fluence). A second, time-delayed (by P1-P2 delay), weak pump pulse $\mathrm{P}_{2}$ (red, narrow) perturbs the excited state. The probe pulse (green) monitors the response of the excited state following photoexcitation. $\mathrm{b}$ ) Coherent control of the $5.7 \mathrm{THz}$ phonon mode in the monoclinic phase of $\mathrm{VO}_{2}$ : Top: Transient response induced by $\mathrm{P} 1$ alone (below threshold) and probed using $525 \mathrm{~nm}$ laser pulses analogous to Fig. 17b). Bottom: Transient reflectivity changes following P1-P2 excitation at different time delays. This corresponds to a tuning between in-phase and out-of-phase excitation as indicated by the arrows at the top. c) The transient response due to $\mathrm{P} 2$ excitation is extracted by subtraction of the P1 response (black curve in b)). 
but shifted in time by the respective P1-P2 delay. The pump-probe response of the below-threshold photoexcited state is therefore exactly the same as the one of the equilibrium monoclinic phase, meaning that the monoclinic symmetry in all cases determines the coherent phonon response.

Ultrafast dynamics in the optical response of the transient phase. The situation is entirely different when driving the photoinduced phase transition by the first pump pulse P1: From the photoemission experiments (section 3.3), it is known that the band gap collapses instantaneously with photoexcitation and that the carriers relax on a 200 fs timescale. It will be shown in the following that monoclinic phase phonons can neither be excited nor coherently controlled anymore, demonstrating that the monoclinic phase symmetry has been lost instantaneously after photoexcitation, i.e. on similar timescales as the insulating gap disappearance. Moreover, the optical response of the transient phase starts to resemble the one of the equilibrium metallic, rutile phase only after $200 \mathrm{fs}$ when the excited carriers have relaxed.

The first excitation by $\mathrm{P} 1$ is now done with a fluence above the photoinduced phase transition threshold $\left(F_{\mathrm{P} 1}=\right.$

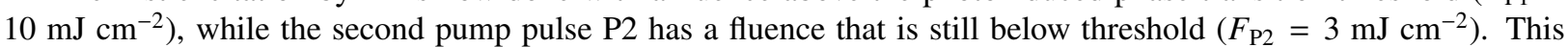
scheme represents a weak perturbation by $\mathrm{P} 2$ of the transient phase, created by $\mathrm{P} 1$. The transient reflectivity change due to P1 and P2 is separately shown in Fig. 19a): The below-threshold pump P2 alone (light blue) excites coherent phonon oscillations, as the monoclinic phase symmetry is not lost. The pump pulse P1, on the contrary, has a sufficient fluence to drive the transition; therefore, no coherent phonon oscillations are present (black). Also, further transient reflectivity changes are shown: First exciting with P1 and later, after a P1-P2 delay of 88 (red) and 176 fs (green), weakly perturbing the transient phase excited by P2. The two delays correspond to out-of-phase $(\pi)$ and in-phase $(2 \pi)$ excitation with respect to the monoclinic phase $5.7 \mathrm{THz}$ phonon mode and are indicated by the arrows. The reflectivity drops further after excitation by the second pump pulse P2 and reaches a similar level around $800 \mathrm{fs}$ after the first excitation by P1. The onset of the additional drop is obviously delayed by the P1-P2 delay, but no phonon oscillation is observed after the second, weak pump pulse P2.

The delay between $\mathrm{P} 1$ and $\mathrm{P} 2$ is now varied systematically to probe the dynamics of the transient phase at different times after its creation: Transient reflectivity changes initiated by $\mathrm{P}^{22}$ are shown in Fig. 19b) with respect to the arrival of the second pump pulse (P2) for direct comparison. At time zero (corresponding to P2's arrival time) the falling edge, marked with a red arrow and the letter A, looses its steepness with increasing P1-P2 delay. Simultaneously, a peak-like structure, marked with a red arrow and the letter B, starts to develop and increases with increasing P1-P2 delay. To further analyse the evolution of feature B, the transients from $\mathrm{b}$ ) are displayed in c) with respect to the arrival of the initial pump P1 and offset along the $\Delta R$ axis. The regions where the signal becomes positive are marked in red, and in-phase and out-of-phase P1-P2 delays are indicated by the thick lines and the markers ( $\pi$ and $2 \pi)$. Additionally, on the right hand side of c) the response induced by P2 is shown for a P1-P2 delay of $\sim 5$ ps (red curve). It clearly resembles the shape of the pump-induced response of the equilibrium metallic phase at high temperatures, which was already shown in Fig. 15 and is plotted again in Fig. 19c) (dashed black curve) for comparison.

When both pump pulses arrive shortly after each other, the non-thermal character of the phase transition is enhanced. Increasing the delay between the two excitations, regions in the transient phase are excited by P2 to an increasing extent, leading to a superposition of the response characteristic for the PIPT itself and the response of $\mathrm{VO}_{2}$ in the transient phase. At none of the delays, P2 excites any coherent signal, showing that the monoclinic lattice potential symmetry has been lost. Instead, it seems highly likely that the positive intensity B evolving with increasing P1-P2 time delay as well as the change of slope (feature A) is related to the build-up of metallic rutile-like optical properties of photoexcited $\mathrm{VO}_{2}$.

A quantitative analysis of the appearance of the peak-like (i.e. equilibrium metallic phase-like) response with respect to the P1-P2 delay is shown in Fig. 19d): Here, the positive sign of the transient change of reflectivity (as opposed to the negative sign of all other optical resonses) is used as a marker for the appearance of a response that is similar to the one in the equilibrium metallic phase. Integration of the transients in c) over a 50 fs window, centred around the arrival time of P2, yields the peak response of feature B as a function of P1-P2 delay in Fig. 19d) (green curve). The integrated intensity is first negative and starts to become positive at a P1-P2 delay time of 160 fs. An additional integration window of $100 \mathrm{fs}$ width (red curve) centred around the arrival time of P2 was used to show

\footnotetext{
${ }^{22}$ The P1-only transient, shown in Fig. 19 a) has been subtracted from the raw data leaving only the changes created by P2.
} 

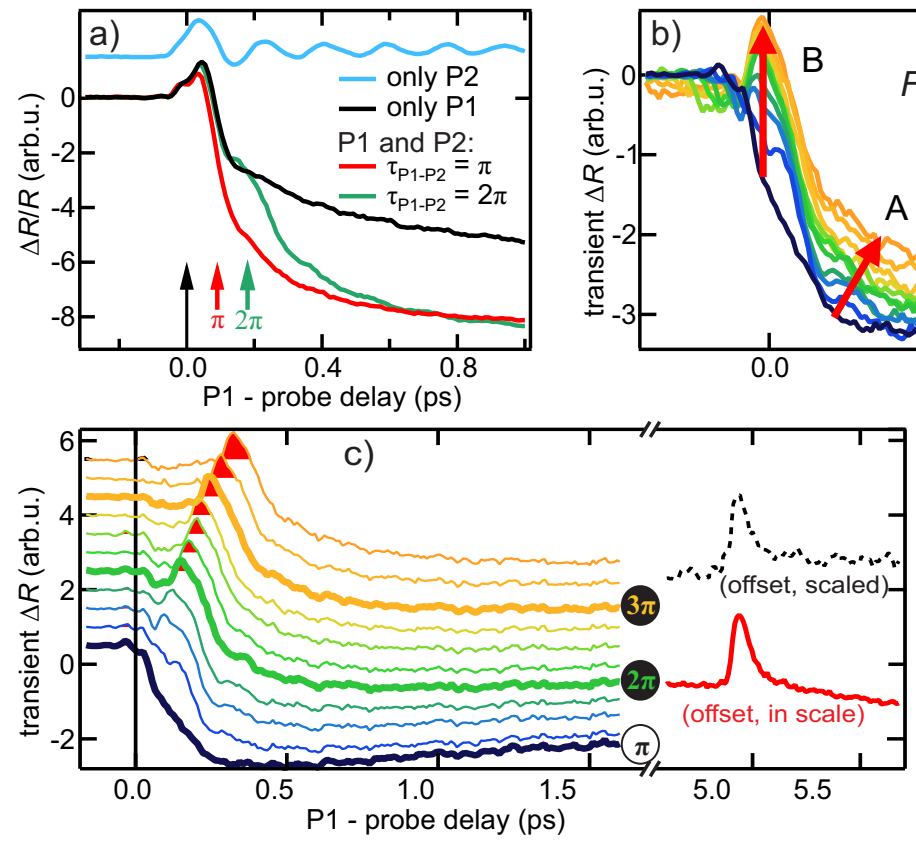
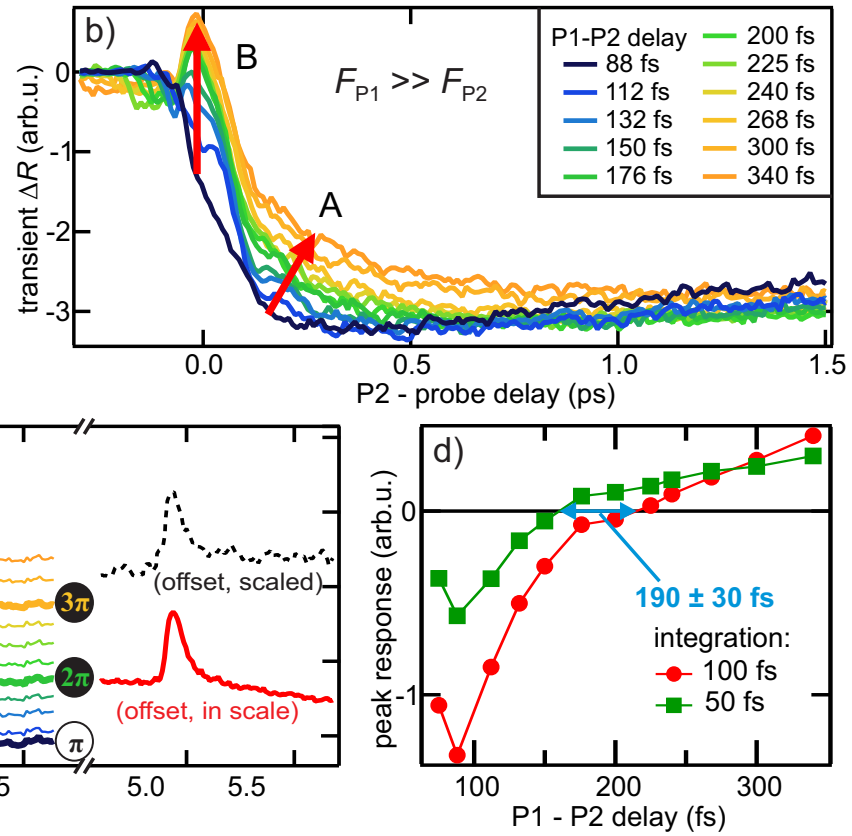

Figure 19: Probing the dynamics of the strongly photoexcited, transient phase: a) shows the response to weak, below threshold perturbation by P2 alone (light blue), the response after inducing the PIPT (black) by pump P1 alone and the perturbation (by P2) of the transient phase in- (green) and out-of-phase (red) with respect to the $5.7 \mathrm{THz}$ phonon oscillation. Subtracting the response caused by P1 alone yields the response of the transient phase shown in b) with respect to the arrival time of P2 for different P1-P2 delays. Arrows indicate changes with P1-P2 delay. In c), the same data as in b) is displayed, but with with respect to the arrival time of P1. For comparison the transient phase response at long P1-P2 delays (5 ps, red curve) and the response of the equilibrium metallic phase (dashed black curve) is also shown. The evolution of feature B in b) with respect to $\mathrm{P} 1-\mathrm{P} 2$ delay is quantified in $\mathrm{d}$ ). 
the robustness of this analysis. ${ }^{23}$ The two integration windows generate very similar curves (red and green), but the P1-P2 delay at which the sign change is observed is larger for the larger integration window (red curve). This can be rationalized by considering that the probe pulse monitores a superposition of P1 and P2 induced changes [82]. The average value of the sign change from this analysis is $190 \pm 30 \mathrm{fs}$ and is, thus, on the same order of magnitude as the mean hot electron/hole relaxation times in the transient phase (section 3.3) and the non-thermal melting of the V-dimers observed by UED (300 fs) [6], suggesting that either or both of these processes are necessary conditions for an optical response of photoexcited $\mathrm{VO}_{2}$ as in its equilibrium metallic rutile phase. As discussed in section 4.2, the short-lived spike in the optical response of the high-temperature phase could result from an overdamped phonon in this structure; it seems reasonable that both, dimer melting and free carrier relaxation must have occured before such optical signature can be established.

\subsection{Summary: Quasi-instantaneous loss of potential symmetry in photoexcited $\mathrm{VO}_{2}$}

The ultrafast dynamics in the optical response of the transient phase after strong photoexcitation are fundamentally different from the ones in the equilibrium monoclinic and rutile phases. Features A and B, shown in Fig. 19 change continuously with P1-P2 delay, in sharp contrast to the observations for the below threshold coherent control experiments where in- and out-of-phase pumping by $\mathrm{P} 2$ lead to a coherent control of one of the monoclinic coherent phonon mode amplitudes. This observation confirms that the monoclinic insulating phase of $\mathrm{VO}_{2}$ is lost on an ultrafast timescale as its symmetry and thereby characteristic underdamped phonon modes have vanished. The lattice potential change occurs on a timescale $\tau_{\mathrm{LPC}}$, shorter than the laser pulse that is driving the transition at sufficiently high fluences.

Fig. 20 illustrates the conclusions from the above analysis: The potential symmetry and resulting forces on the ions (black and red springs and frequencies $\omega_{1 / 2}$ ) of the initial monoclinic phase (blue) is lost immediately, as the insulating band gap, when entering the transient phase (green, higher symmetry lattice potential is illustrated by the dark blue springs). However, the instant vanishing of the monoclinic symmetry of the lattice potential does not imply that the transient phase of $\mathrm{VO}_{2}$ is equal to its metallic, rutile equilibrium phase (orange). On the contrary, the transient response only slowly starts to resemble that of the equilibrium rutile phase after hundreds of fs: Its shape becomes similar after picoseconds have passed, a time after excitation when the atoms are still far from their rutile atomic positions as shown by previous time-resolved diffraction experiments [6, 57-60]. This means that initially, right after P1 drives the system out of the monoclinic potential symmetry, and before significant ionic motion has occured $\left(\tau_{\mathrm{CPT}} \gg \tau_{\mathrm{LPC}}\right)$, a non-thermal, metallic state far from equilibrium is established. Whether the LPC is a direct consequence of the bonding dimer orbitals or whether it results from the enhanced screening caused by the instantaneous IMT, cannot be deduced from the presented experiments, as these processes occur faster than the available time resolution. However, clearly, the transient metallic state does not exhibit the optical response of the equilibrium metallic rutile phase before the hot carriers have relaxed and the V-dimers have melted, suggesting that at least either process is necessary to establish similar dynamics as in the high-temperature phase.

\section{Discussion: A comprehensive picture of the photoinduced, ultrafast phase transitions in $\mathrm{VO}_{2}$}

Fig. 21 summarizes the series of processes occuring during the PIPT of vanadium dioxide. Initially in its lowtemperature equilibrium phase, monoclinic and insulating (left), photoexcitation of $\mathrm{VO}_{2}$ above a certain critical fluence first leads to a change of population of electronic states, creating a highly non-equilibrium plasma. This signifcantly changes the screening of the Coulomb interaction through, mainly, the depopulation of localized, top-VB states. The resulting band gap renormalization leads to a collapse of the insulating band gap, rendering $\mathrm{VO}_{2}$ metallic. The non-thermal IMT has occured. At the same time, probably the depopulation of the V-V bonding orbitals leads to a lattice potential change, as the new charge distribution changes the forces that act on the ions. The monoclinic potential symmetry is lost instantaneously with photoexcitation, representing the trigger for the CPT, which evolves over multiple subsequent timescales.

\footnotetext{
${ }^{23}$ The pump pulse (P2) duration was estimated to be 50 fs. To make sure that a wrong estimate does not change the resulting analysis, integration windows of one and two times the P2 duration were employed. The latter can be seen as a worst case estimate.
} 


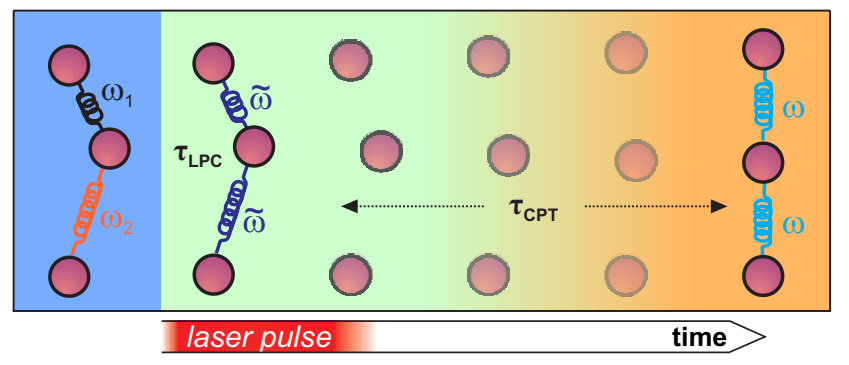

Figure 20: Illustration of the ultrafast symmetry loss of monoclinic $\mathrm{VO}_{2}$ : The lower symmetry monoclinic phase (blue) is photoexcited. The lattice potential changes its symmetry immediately, i.e. monoclinic phonon modes vanish. Starting at this highly non-equilibrium transient phase (green), the positions of the atoms (red circles), i.e. the actual crystallographic structure, progresses on a much slower timescale $\tau_{\mathrm{CPT}} \gg \tau_{\mathrm{LPC}}$ until it stabilises the metallic rutile phase (orange).

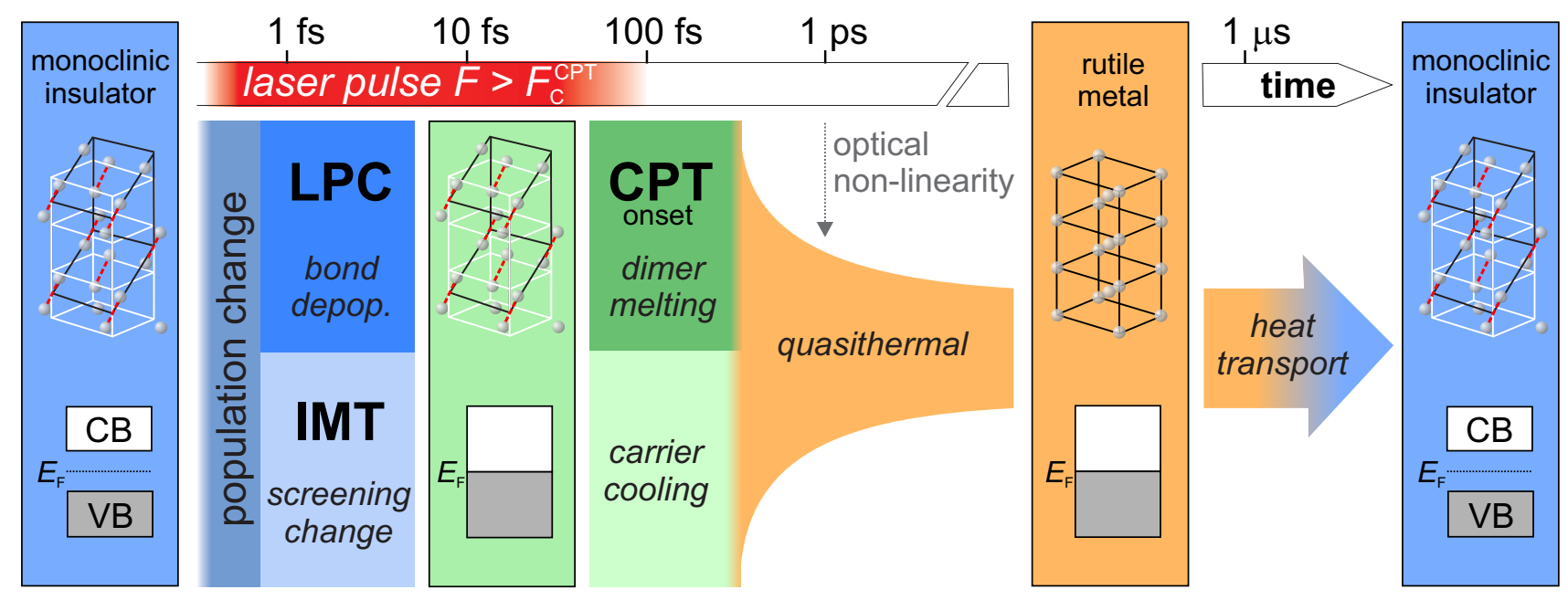

Figure 21: Photoinduced phase transitions of, initially, monoclinic insulating $\mathrm{VO}_{2}$ above $F_{C}^{C P T}$ excitation. The population change created by photoexcitation changes the screening of the CIA leading to the IMT on screening timescales (few fs), i.e. instantaneous with the optical excitation using $\sim 60 \mathrm{fs}$ laser pulses [5]. As the V-V bonding orbitals are depopulated by photoexciation [5], the LPC is initiated on the same timescales [83]. The resulting transient phase of vanadium dioxide is, at first, a highly excited metal of monoclinic atom arrangement; however, the modified lattice potential drives the ions into new equilibrium conditions. The melting of the V-dimers [6] happens concurrently with the relaxation of excited electrons and holes in the metallic band structure [5], which is accompanied by energy transfer to the lattice. The fluence threshold of optical experiments is usually determined after these processes have occured. Then, the system evolves quasi-thermally to its high-temperature equilibrium phase, before it relaxes back to its monoclinic insulating state when sufficient heat has been transported away. 
The initial transient phase is characterized by a metallic band structure and monoclinic atomic arrangement in a non-monoclinic lattice potential, i.e. at the onset of structural rearrangement. At this point in time, $\mathrm{VO}_{2}$ is in a highly non-equilibrium state and stands at the beginning of a chain of relaxation processes. The electronic system, with its non-thermal carrier distribution, is inclined to thermalize by electron-electron scattering and coupling to phonons. The lattice, in its monoclinic ion arrangement and experiencing forces towards new potenial minima is about to evolve to the rutile structure via a complex pathway and a multitude of ionic motions. Probably the first step of electronic and lattice relaxation occurs concurrently within 200 and $300 \mathrm{fs}$, respectively: The excited carriers decay towards $E_{F}$ and also the V-dimers melt, destroying the periodic lattice distortion along the $\mathrm{VO}_{2} \mathrm{c}$-axis [6]. The concurrency of these processes suggests an interconnection between the two, which, however, cannot be unambiguously concluded from the presented results. Only after both processes have happened, the transient optical response of photoexcited $\mathrm{VO}_{2}$ resembles the one of the high-temperature phase. This strongly suggests that at least one of these processes is necessary condition for the metal-like time-dependent optical properties.

After the carrier thermalization, the electronic structure does not expose any dynamics in the time-resolved PES experiments while the lattice continues to evolve [6, 57-60]. The excess energy of electrons and holes after photoexcitation has been transferred to the lattice to a large extent, bringing the subsystems into thermal equilibrium. The crystal structure evolves towards the rutile arrangement and, at even later times when heat transport cools the sample down, eventually leads to a restoration of the monoclinic and insulating, low-temperature phase.

The above-discussed dynamics of the PIPT occur only if the initial photoexcitation using $800 \mathrm{~nm}$ light is sufficiently strong. Quantification of the required strength is, however, a tidious task and has been approached in multiple ways in the past. Threshold fluences for the occurence of different characteristic processes and observables have been defined on the basis of various experiments, however, due to the complexity of photoinduced phenomena in $\mathrm{VO}_{2}$, their comparison needs to be treated with great caution. This difficulty is further complicated by the experimental challenges of accurate fluence determination, different probing volumes of different techniques, and variations between different types of $\mathrm{VO}_{2}$ samples, which - when conservatively judged - only provides comparability of threshold fluences by order of magnitude. In the following list, we discuss the meaning and comparability of some of the fluence thresholds published in the past on the basis of the picture of the PIPT developed above:

- Ultrafast electron diffraction: The threshold fluences determined in UED experiments [6, 57] are probably the "cleanest" thresholds available, as they are extracted from a direct observable of the CPT, the diffraction signatures of the crystal lattice. They also exhibit a certain sensitivity to charge redistribution through their dependence on the atomic form factors. Furthermore, they provide information on how strong the photoexcitation needs to occur to lead to a particular rearrangement. With $F_{\mathrm{C}}=9 \mathrm{~mJ} / \mathrm{cm}^{2}$ for the V-dimer melting and an extrapolated saturation fluence (i.e. the whole probed volume is transformed) of $F_{\text {sat }}=43 \mathrm{~mJ} / \mathrm{cm}^{2}[6]$, these results define a critical excitation density needed to initiate this early step of the CPT.

- Transient optical spectroscopy: The significant number of optical studies on the PIPT provides us with almost an equal number of threshold fluences. For the sake of simplicity and because we are convinced that the absolute values for threshold fluences have only a very limited meaning, we focus here on a discussion of the determination procedure. Historically, $F_{\mathrm{C}}$ is determined at a delay of $1 \mathrm{ps}$ after optical excitation. Due to the long lifetime of the optical response, the same values could be extracted at larger delays, e.g. at 3.5 ps as demonstrated in Ref. [82]. Based on the results presented above, it can be concluded that - at 1 ps - all non-thermal processes have occured, as illustrated by Fig. 21. The non-linearity in the optical response at 1 ps, thus, trivially, only shows that $F_{C}$ is sufficient to launch all processes necessary to cause this non-linearity and that the excited state has a lifetime larger than 1 ps. However, as optical spectroscopy is sensitive to the dielectric function and, therefore, to contributions of lattice and electronic system, this observable is simply not capable of distantangling which of the preceding processes (LPC, CPT, IMT, or carrier relaxation, cf. Fig. 21) is responsible for the non-linearity. We emphasize this, as either structural or electronic branch of the PIPT after above- $F_{\mathrm{C}}$ excitation may well have a lower fluence threshold that is simply not reflected in the optical experiments. The results presented in this article yield $F_{\text {sat }} \sim 4-5 \times F_{\mathrm{C}}$, coinciding with the UED ratio. This agreement suggests that both experimental observables, the non-linearity of the optical response at 1 ps and the V-dimer melting, have the same origin.

- Coherent phonon spectroscopy: As shown in section 4.3, the coherent phonons of the monoclinic structure 
expose the same $F_{\mathrm{C}}$ and $F_{\text {sat }}$ as the optical response at $1 \mathrm{ps}$. The coherent phonon dynamics already occur at time delays before $1 \mathrm{ps}$, again showing that - whatever causes the non-linearity in the optical response has its origin in dynamics happening at earlier times. Moreover, the coherent phonons were used to show that the symmetry of the lattice potential is lost instantaneously with photoexciation (section 4.4), and it is highly probable that this LPC is the origin of the subsequent lattice dynamics like the melting of the V-dimers. This view is further supported when considering that hole doping to the $\mathrm{V}$-V bonding orbitals, which is the main driving mechanism of the band gap collapse (see section 3.3), will likely have and impact on the lattice potential. These considerations strongly suggest that, in fact, the critical fluence observed in optical and coherent phonon spectroscopy relates to the excitation density necessary to change the lattice potential symmetry, i.e. causing first the LPC and, consequently, the CPT. This value should probably be, in agreement with previous observations [94], independent of the pump photon energy (if above gap) and depend on the number of carriers that are being redistributed through photoexcitation. If and how this $F_{\mathrm{C}}=F_{\mathrm{C}}^{\mathrm{CPT}}$ is connected to the concurrent IMT remains unknown. In particular, it is well possible that the IMT already occurs for lower fluences $\left(F_{\mathrm{C}}^{\mathrm{IMT}}<F_{\mathrm{C}}^{\mathrm{CPT}}\right)$.

- Time-dependent multiterahertz spectroscopy: When probing the dielectric function at $\mathrm{THz}$ frequencies, a similar argumentation as the one for optical spectroscopy holds: Sensitive to the dielectric function, this technique cannot directly disentangle between increased conductivity due to excited carriers and the creation of DOS at a material's equilibrium Fermi level. However, it should be noted that, at these long wavelengths, the transient response is very sensitive to low-energy excitations of (quasi-)free carriers as discussed in section 1.2. These can be subject to other non-linearities like the formation of an electron-hole plasma at excitation densities larger than the Mott limit. ${ }^{24}$ In principle, such delocalization of carriers (or even the IMT, as discussed above) could occur also at excitation fluences that are lower than the $F_{\mathrm{C}}^{\mathrm{CPT}}$ needed to cause the non-linearity of the $1 \mathrm{ps}$ at optical frequencies, i.e. for lower excitation densities than the ones required to drive the loss of monoclinic symmetry.

- Time-resolved photoelectron spectroscopy: Complementary to the above discussed techniques, PES is directly sensitive to the changes of the electronic structure. Thus, theoretically, this technique could provide a fluence threshold for the pure IMT, analogously to the CPT threshold determined by UED. Unfortunately, experimental difficulties significantly limit the range of pump fluences that can be used in photoemission (see also section 2.2). ${ }^{25}$ Fortunately, in time-resolved PES, it is not necessary to give evidence for non-linear behaviour in order to demonstrate that the IMT has occured; the bare observation of photoinduced DOS in the insulating band gap is sufficient proof. Yet, further studies at lower excitation densities, preferrably with even higher time resolution and on single crystal surfaces would be highly desirable for an unambiguous determination of the critical fluence of the IMT.

The above results and discussion provide strong evidence for a similar cause of the critical fluences determined by UED, optical, and coherent phonon spectroscopy. Given the structural origin of the dynamics probed by coherent phonon spectroscopy and UED, which are sensitive to the photoinduced LPC and CPT, respectively, it seems highly probable that the non-linearity of the optical response 1 ps after photoexcitation, which is used to determine the critical fluence in optical experiments, has the same source and, thus, is a consequence of the LPC followed by the CPT. We are convinced that, from the currently available data, it is impossible to decide if the electronic branch of of the PIPT (bottom of Fig. 21) requires the same excitation density or not. In particular, it is well possible that the IMT can be launched using lower fluences $F_{\mathrm{C}}^{\mathrm{IMT}}<F<F_{\mathrm{C}}^{\mathrm{CPT}}$, i.e. without initiating the chain of structural processes. For example time-resolved PES experiments at excitation densities clearly below $F_{\mathrm{C}}^{\mathrm{CPT}}$ could elucidate if a band gap collapse can be observed in the absence of the structural transition. However, already today the low fluence threshold in $\mathrm{mTHz}$

\footnotetext{
${ }^{24}$ The Mott limit is defined as the excitation density at which the Bohr radii of electrons and holes start overlapping and many body phenomena start dominating the dynamics.

${ }^{25}$ Reduced heat transport due to the required UHV conditions sets an upper limit to the tolerable energy deposition in high repetition rate experiments that can only partially be compensated by sample cooling, as the reduced temperature outside of the laser spot reduces the conductivity and leads to charging through the photoemission process. Also, multiphoton-photoemission through the pump photons increases non-linearly with rising excitation density. Finally, low fluence investigations are, even at high repetition rates ( $40 \mathrm{kHz})$ hardly realizable due to the small signal-to-background ratio.
} 


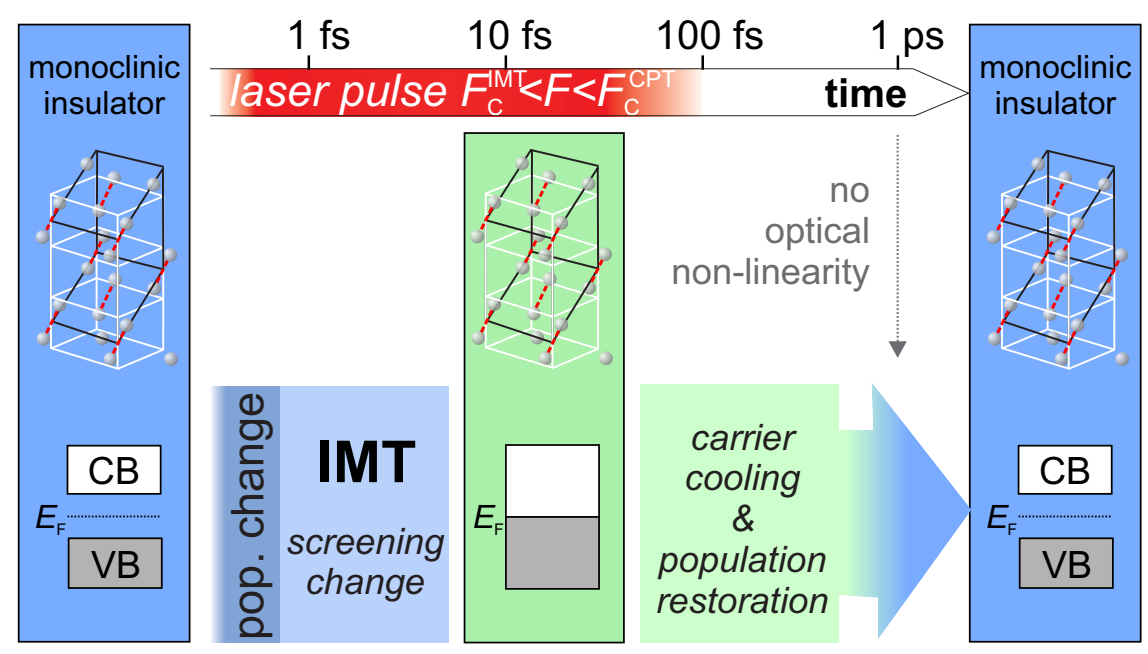

Figure 22: Possible scenario for a non-thermal insulator-to-metal transition in $\mathrm{VO}_{2}$ and back relaxation on ultrafast timescales for optical excitation below the critical fluence of the structural phase transition. The population change modifies the screening of the CIA, leading to a band gap collapse on screening timescales. In the absencence of stabilization of the transient phase through structural reorganization, the insulating band gap will open when the excited carriers relax to their ground state energy levels.

spectroscopy [8, 48] as well as the second threshold fluence in UED experiments [6] (see section 1.2 for an overview) point at complex dynamics below $F_{\mathrm{C}}^{\mathrm{CPT}}$.

Fig. 22 illustrates the case of a fully independent IMT for fluences below $F_{\mathrm{C}}^{\mathrm{CPT}}$. For example, no concurrent dimer-melting would accompany the carrier relaxation in the metallic band structure. This means that the metallicity of the transient phase could not be stabilized by the structural dynamics. Electrons and holes would recombine and restore the ground state population of VB orbitals and, as the vanadium atoms remain paired, this recombination would reduce the screening of the CIA and re-establish its original strength. This would lead to a reopening of the gap. The transiently metallic state would decay on ultrafast timescales determined by the electron-hole recombination and not, as for $F>F_{\mathrm{C}}^{\mathrm{CPT}}$ excitation, on timescales dictated by heat transport. The observation of such a short-lived, transiently metallic state of monoclinic $\mathrm{VO}_{2}$ would be the unambiguous proof of a purely electronic origin of the IMT.

\section{Summary and conclusions}

In summary, we have shown that the population change of electronic levels and resulting highly non-equilibrium plasma caused by above-threshold photexcitation of monoclinic, insulating $\mathrm{VO}_{2}$ stands at the very beginning of both, a structural and an electronic phase transition. On the one hand, it is the origin of a significant change of the screening of the CIA that instantaneously leads to a collapse of the band gap, which is followed by the relaxation of highly excited carriers on a $200 \mathrm{fs}$ timescale. On the other hand, probably the emptying of bonding orbitals of the V-dimers that are characteristic of the monoclinic phase results in an instantaneous lattice potential change that stands at the beginning of the CPT.

Based on the results discussed, we conclude that photoexcitation of $\mathrm{VO}_{2}$ above a threshold fluence $F_{\mathrm{C}}^{\mathrm{CPT}}$ leads to a charge redistribution that changes (i) the screening of the CIA sufficiently strong to drive the IMT and (ii) the lattice potential symmetry, which is the origin of the CPT occuring on longer timescales (cf. Fig. 21). This, first of all and maybe trivially, means that a purely electronic process lies at the heart of both PIPTs in $\mathrm{VO}_{2}$ when excited by $800 \mathrm{~nm}$ laser pulses. It also shows that the "detour" via a lattice rearrangement is not necessary to reach a metallic phase. The transient phase is, initially, a crystallographic monoclinic metal and ionic motion not a necessary condition for the IMT. The fact that the IMT is caused by charge redistribution is also fully consistent with most recent results on THz-pumping of $\mathrm{VO}_{2}$ [71], which showed that the strong electric field of the incident pump pulse perturbs the potential energy landscape such that electrons tunnel from top VB states to normally unoccupied ones. The purely electronic origin of the PIPTs of course does not mean that the lattice does not respond or only responds thermally to 
the photoexcitation. It is very likely that the lattice plays an important role in stabilising the metallic phase established on screening timescales. This could either occur by non-thermal melting of the V-dimers or due to the increased temperature after thermalisation of the electronic subsystem with the lattice.

From our point of view, the provocative, simplifying question of Wentzcovitch et al. from 1992 "VO $\mathrm{VO}_{2}$ : Peierls or Mott-Hubbard?" [19] should, based on the findings of recent ultrafast studies, be replaced and refined: Sufficiently strong optical excitation launches two branches of dynamics, (i) a structural one, which begins with a an instantaneous lattice potential change followed by the actual ionic motion, and (ii) an electronic one, which starts with an instantaneous insulator-to-metal transition followed by charge carrier relaxation. The early steps of both pathways occur concurrently. The initiation is, in both cases, the photoinduced change of population of the electronic levels in the band gap region, i.e. a purely electronic process. If an electronic process is the origin of the structural branch of the PIPT, what do we consider to be the beginning of the structural dynamics? The lattice potential change or the beginning of ionic motion through the non-thermal melting of the V-dimers? Would an electronic transition that is caused by structural dynamics be determined by the timescales of the LPC or of ionic motion? -An unambiguous proof of fully independent IMT could, thus, be provided by future experiments with enhanced time resolution providing insight into the dynamics of LPC and IMT on screening timescales. Alternatively, the observation of an insulator-to-metal transition at excitation densities not sufficient to cause the LPC in the first place could serve the same purpose. We conclude that the frequently applied ultrafast approach to disentangle electronic and structural phenomena in the time domain has to be treated with great caution, as structural phenomena may well have their origin in processes ocurring on electronic timescales.

\section{Acknowledgements}

We would like to thank S. Wall, M. Herzog, L. Foglia, and M. Wolf from the Fritz Haber Institute in Berlin, who have contributed to this work in many ways. Particularily, we acknowledge S. Wall for his contribution to the optical and coherent phonon experiments, the data analysis, and scientific discussions. Similarily, we owe thanks to M. Herzog for his work on the photoemission experiment, analysis and discussion. M. Wolf provided valuable scientific intput and infrastructure for the $\mathrm{VO}_{2}$ project, which we greatly acknowledge. We are also thankful to A. Rubio, M. Gatti, L. Xian, and P. Cudazzo as well as R. Haglund, C. McGahan, R. Marvel, and J. Nag for their important contributions and fruitful collaboration. We greatly acknowledge scientific discussions with A. Leitenstorfer and B. Siwick at multiple occasions. D. Wegkamp acknowledges funding by the Helmhotz Graduate School "Dynamics in New Light".

[1] S. Westman, Note on a Phase Transition in $\mathrm{VO}_{2}$, Acta Chem. Scand. 15 (1961) 217.

[2] F. J. Morin, Oxides Which Show a Metal-to-Insulator Transition at the Neel Temperature, Phys. Rev. Lett. 3 (1959) 34-36, doi: 10.1103/PhysRevLett.3.34, URL http://link.aps.org/doi/10.1103/PhysRevLett.3.34.

[3] W. Roach, I. Balberg, Optical induction and detection of fast phase transition in VO $\mathrm{V}_{2}$, Solid State Commun. 9 (9) (1971) $551 \quad-\quad 555, \quad$ doi:http://dx.doi.org/10.1016/0038-1098(71)90144-X, URL http://www.sciencedirect.com/science/article/pii/003810987190144X.

[4] S. Wall, D. Brida, S. R. Clark, H. P. Ehrke, D. Jaksch, A. Ardavan, S. Bonora, H. Uemura, Y. Takahashi, T. Hasegawa, H. Okamoto, G. Cerullo, A. Cavalleri, Quantum interference between charge excitation paths in a solid-state Mott insulator, Nat. Phys. 7 (2) (2011) 114-118, URL http://dx.doi.org/10.1038/nphys1831.

[5] D. Wegkamp, M. Herzog, L. Xian, M. Gatti, P. Cudazzo, C. L. McGahan, R. E. Marvel, R. F. Haglund, A. Rubio, M. Wolf, J. Stähler, Instantaneous Band Gap Collapse in Photoexcited Monoclinic $\mathrm{VO}_{2}$ due to Photocarrier Doping, Phys. Rev. Lett. 113 (2014) 216401, doi: 10.1103/PhysRevLett.113.216401, URL http://link.aps.org/doi/10.1103/PhysRevLett.113.216401.

[6] V. R. Morrison, R. P. Chatelain, K. L. Tiwari, A. Hendaoui, A. Bruhács, M. Chaker, B. J. Siwick, A photoinduced metal-like phase of monoclinic $\mathrm{VO}_{2}$ revealed by ultrafast electron diffraction, Science 346 (6208) (2014) 445-448, doi:10.1126/science.1253779, URL http://www.sciencemag. org/content/346/6208/445. abstract.

[7] S. Wall, L. Foglia, D. Wegkamp, K. Appavoo, J. Nag, R. F. Haglund, J. Stähler, M. Wolf, Tracking the evolution of electronic and structural properties of $\mathrm{VO}_{2}$ during the ultrafast photoinduced insulator-metal transition, Phys. Rev. B 87 (2013) 115126, doi: 10.1103/PhysRevB.87.115126, URL http://link.aps.org/doi/10.1103/PhysRevB.87.115126.

[8] C. Kübler, H. Ehrke, R. Huber, R. Lopez, A. Halabica, R. F. Haglund, A. Leitenstorfer, Coherent Structural Dynamics and Electronic Correlations during an Ultrafast Insulator-to-Metal Phase Transition in $\mathrm{VO}_{2}$, Phys. Rev. Lett. 99 (2007) 116401, doi: 10.1103/PhysRevLett.99.116401, URL http://link.aps.org/doi/10.1103/PhysRevLett.99.116401.

[9] J. M. Longo, P. Kierkegaard, A Refinement of the Structure of $\mathrm{VO}_{2}$, Acta Chem. Scand. 24 (1970) 420.

[10] D. B. McWhan, M. Marezio, J. P. Remeika, P. D. Dernier, X-ray diffraction study of metallic VO 2 , Phys. Rev. B 10 (1974) 490-495, doi:10.1103/PhysRevB.10.490, URL http://link.aps.org/doi/10.1103/PhysRevB.10.490. 
[11] D. Kucharczyk, T. Niklewski, Accurate X-ray determination of the lattice parameters and the thermal expansion coefficients of $\mathrm{VO}_{2}$ near the transition temperature, J. Appl. Crystallogr. 12 (4) (1979) 370-373, doi:10.1107/S0021889879012711, URL http://dx.doi.org/10.1107/S0021889879012711.

[12] V. Eyert, The metal-insulator transitions of $\mathrm{VO}_{2}$ : A band theoretical approach, Ann. Phys. 11 (9) (2002) 650-704, doi:10.1002/1521-3889(200210)11:9;650::AID-ANDP650 $3.0 . C O ; 2-K$ URL http://dx.doi.org/10.1002/1521-3889(200210) 11:9<650: :AID-ANDP650>3.0.C0;2-K.

[13] P. Schilbe, Raman scattering in $\mathrm{VO}_{2}$, Physica B 316 - 317 (0) (2002) 600 - 602, proceedings of the 10th International Conference on Phonon Scattering in Condensed Matter.

[14] A. C. Jones, S. Berweger, J. Wei, D. Cobden, M. B. Raschke, Nano-optical Investigations of the Metal-Insulator Phase Behavior of Individual $\mathrm{VO}_{2}$ Microcrystals, Nano Lett. 10 (5) (2010) 1574-1581, doi:10.1021/n1903765h, URL http://pubs.acs.org/doi/abs/10.1021/n1903765h.

[15] C. N. Berglund, H. J. Guggenheim, Electronic Properties of $\mathrm{VO}_{2}$ near the Semiconductor-Metal Transition, Phys. Rev. 185 (1969) $1022-$ 1033, doi:10.1103/PhysRev.185.1022, URL http://link.aps.org/doi/10.1103/PhysRev.185.1022.

[16] J. B. Goodenough, The two components of the crystallographic transition in $\mathrm{VO}_{2}$, J. Solid State Chem. 3 (4) (1971) $490 \quad-\quad 500, \quad$ doi:http://dx.doi.org/10.1016/0022-4596(71)90091-0, URL http://www.sciencedirect.com/science/article/pii/0022459671900910.

[17] A. Zylbersztejn, N. F. Mott, Metal-insulator transition in vanadium dioxide, Phys. Rev. B 11 (1975) 4383-4395, doi: 10.1103/PhysRevB.11.4383, URL http://link.aps.org/doi/10.1103/PhysRevB.11.4383.

[18] N. F. Mott, L. Friedman, Metal-insulator transitions in $\mathrm{VO}_{2}, \mathrm{Ti}_{2} \mathrm{O}_{3}$ and $\mathrm{Ti}_{2-x} \mathrm{~V}_{x} \mathrm{O}_{3}$, Philos. Mag. 30 (2) (1974) 389-402, doi: 10.1080/14786439808206565.

[19] R. M. Wentzcovitch, W. W. Schulz, P. B. Allen, VO 2 : Peierls or Mott-Hubbard? A view from band theory, Phys. Rev. Lett. 72 (1994) 3389-3392, doi:10.1103/PhysRevLett.72.3389, URL http://link.aps.org/doi/10.1103/PhysRevLett.72.3389.

[20] M. A. Korotin, N. A. Skorikov, V. I. Anisimov, Variation of orbital symmetry of the localized $3 d^{1}$ electron of the $\mathrm{V}^{4+}$ ion upon the metalinsulator transition in $\mathrm{VO}_{2}$, arXiv:cond-mat/0301347v1 [cond-mat.str-el] .

[21] V. Eyert, $\mathrm{VO}_{2}$ : A Novel View from Band Theory, Phys. Rev. Lett. 107 (2011) 016401, doi:10.1103/PhysRevLett.107.016401, URL http://link.aps.org/doi/10.1103/PhysRevLett.107.016401.

[22] R. Grau-Crespo, H. Wang, U. Schwingenschlögl, Why the Heyd-Scuseria-Ernzerhof hybrid functional description of $\mathrm{VO}_{2}$ phases is not correct, Phys. Rev. B 86 (2012) 081101, doi:10.1103/PhysRevB.86.081101, URL http://link.aps.org/doi/10.1103/PhysRevB.86.081101.

[23] X. Yuan, Y. Zhang, T. A. Abtew, P. Zhang, W. Zhang, $\mathrm{VO}_{2}$ : Orbital competition, magnetism, and phase stability, Phys. Rev. B 86 (2012) 235103, doi:10.1103/PhysRevB.86.235103, URL http://link.aps.org/doi/10.1103/PhysRevB.86.235103.

[24] J. E. Coulter, E. Manousakis, A. Gali, Limitations of the hybrid functional approach to electronic structure of transition metal oxides, Phys. Rev. B 88 (2013) 041107, doi:10.1103/PhysRevB.88.041107, URL http://link.aps .org/doi/10.1103/PhysRevB.88.041107.

[25] L. Hedin, New Method for Calculating the One-Particle Green's Function with Application to the Electron-Gas Problem, Phys. Rev. 139 (1965) A796-A823, doi:10.1103/PhysRev.139.A796, URL http://link.aps.org/doi/10.1103/PhysRev.139.A796.

[26] A. Continenza, S. Massidda, M. Posternak, Self-energy corrections in $\mathrm{VO}_{2}$ within a model GW scheme, Phys. Rev. B 60 (1999) 1569915704, doi:10.1103/PhysRevB.60.15699, URL http://link.aps .org/doi/10.1103/PhysRevB.60.15699.

[27] S. Biermann, A. Poteryaev, A. I. Lichtenstein, A. Georges, Dynamical Singlets and Correlation-Assisted Peierls Transition in $\mathrm{VO}_{2}$, Phys. Rev. Lett. 94 (2005) 026404, doi:10.1103/PhysRevLett.94.026404, URL http://link.aps.org/doi/10.1103/PhysRevLett.94.026404.

[28] M. Gatti, Correlation effects in valence-electron spectroscopy of transition-metal oxides: many-body perturbation theory and alternative approaches, Ph.D. thesis, L'École Polytechnique, 2007.

[29] M. Gatti, F. Bruneval, V. Olevano, L. Reining, Understanding Correlations in Vanadium Dioxide from First Principles, Phys. Rev. Lett. 99 (2007) 266402, doi:10.1103/PhysRevLett.99.266402, URL http://link .aps.org/doi/10.1103/PhysRevLett.99.266402.

[30] W. H. Brito, M. C. O. Aguiar, K. Haule, K. G., Metal-insulator Transition in VO2: a DFT+DMFT perspective, arXiv:1509.02968

[31] A. Bianconi, S. Stizza, R. Bernardini, Critical behavior of the plasmon resonance at the metal-insulator transition in $\mathrm{VO}_{2}$, Phys. Rev. B 24 (1981) 4406-4411, doi:10.1103/PhysRevB.24.4406, URL http://link. aps .org/doi/10.1103/PhysRevB.24.4406.

[32] S. Shin, S. Suga, M. Taniguchi, M. Fujisawa, H. Kanzaki, A. Fujimori, H. Daimon, Y. Ueda, K. Kosuge, S. Kachi, Vacuum-ultraviolet reflectance and photoemission study of the metal-insulator phase transitions in $\mathrm{VO}_{2}, \mathrm{~V}_{6} \mathrm{O}_{13}$, and $\mathrm{V}_{2} \mathrm{O}_{3}$, Phys. Rev. B 41 (1990) 4993-5009, doi:10.1103/PhysRevB.41.4993, URL http://link.aps.org/doi/10.1103/PhysRevB.41.4993.

[33] T. C. Koethe, Z. Hu, M. W. Haverkort, C. Schüßler-Langeheine, F. Venturini, N. B. Brookes, O. Tjernberg, W. Reichelt, H. H. Hsieh, H.-J. Lin, C. T. Chen, L. H. Tjeng, Transfer of Spectral Weight and Symmetry across the Metal-Insulator Transition in VO 2 , Phys. Rev. Lett. 97 (2006) 116402, doi:10.1103/PhysRevLett.97.116402, URL http://link .aps.org/doi/10.1103/PhysRevLett.97.116402.

[34] K. Saeki, T. Wakita, Y. Muraoka, M. Hirai, T. Yokoya, R. Eguchi, S. Shin, Band dispersion near the Fermi level for $\mathrm{VO}_{2}$ thin films grown on $\mathrm{TiO}_{2}(001)$ substrates, Phys. Rev. B 80 (2009) 125406, doi:10.1103/PhysRevB.80.125406, URL http://link.aps.org/doi/10.1103/PhysRevB.80.125406.

[35] S. Suga, A. Sekiyama, S. Imada, T. Miyamachi, H. Fujiwara, A. Yamasaki, K. Yoshimura, K. Okada, M. Yabashi, K. Tamasaku, A. Higashiya, T. Ishikawa, $\sim 8 \mathrm{keV}$ photoemission of the metal-insulator transition system $\mathrm{VO}_{2}$, New J. Phys. 11 (10) (2009) 103015, URL http: //stacks. iop.org/1367-2630/11/i=10/a=103015.

[36] J. Laverock, S. Kittiwatanakul, A. A. Zakharov, Y. R. Niu, B. Chen, S. A. Wolf, J. Lu, K. E. Smith, Direct observation of decoupled structural and electronic transitions and an ambient pressure monocliniclike metallic phase of $\mathrm{VO}_{2}$, Phys. Rev. Lett. to be published.

[37] H.-T. Kim, Y. W. Lee, B.-J. Kim, B.-G. Chae, S. J. Yun, K.-Y. Kang, K.-J. Han, K.-J. Yee, Y.-S. Lim, Monoclinic and Correlated Metal Phase in $\mathrm{VO}_{2}$ as Evidence of the Mott Transition: Coherent Phonon Analysis, Phys. Rev. Lett. 97 (2006) 266401, doi: 10.1103/PhysRevLett.97.266401, URL http://link.aps.org/doi/10.1103/PhysRevLett.97.266401.

[38] E. Arcangeletti, L. Baldassarre, D. Di Castro, S. Lupi, L. Malavasi, C. Marini, A. Perucchi, P. Postorino, Evidence of a Pressure- 
Induced Metallization Process in Monoclinic VO 2 , Phys. Rev. Lett. 98 (2007) 196406, doi:10.1103/PhysRevLett.98.196406, URL http://link.aps.org/doi/10.1103/PhysRevLett.98.196406.

[39] Z. Tao, T.-R. T. Han, S. D. Mahanti, P. M. Duxbury, F. Yuan, C.-Y. Ruan, K. Wang, J. Wu, Decoupling of Structural and Electronic Phase Transitions in $\mathrm{VO}_{2}$, Phys. Rev. Lett. 109 (2012) 166406, doi:10.1103/PhysRevLett.109.166406, URL http://link.aps.org/doi/10.1103/PhysRevLett.109.166406.

[40] T. L. Cocker, L. V. Titova, S. Fourmaux, G. Holloway, H.-C. Bandulet, D. Brassard, J.-C. Kieffer, M. A. El Khakani, F. A. Hegmann, Phase diagram of the ultrafast photoinduced insulator-metal transition in vanadium dioxide, Phys. Rev. B 85 (2012) 155120, doi: 10.1103/PhysRevB.85.155120, URL http://link.aps.org/doi/10.1103/PhysRevB.85.155120.

[41] M. M. Qazilbash, M. Brehm, B.-G. Chae, P.-C. Ho, G. O. Andreev, B.-J. Kim, S. J. Yun, A. V. Balatsky, M. B. Maple, F. Keilmann, H.-T. Kim, D. N. Basov, Mott Transition in $\mathrm{VO}_{2}$ Revealed by Infrared Spectroscopy and Nano-Imaging, Science 318 (5857) (2007) 1750-1753, doi:10.1126/science.1150124, URL http: //www. sciencemag. org/content/318/5857/1750. abstract.

[42] A. K. Basak, H. Petek, K. Ishioka, E. M. Thatcher, C. J. Stanton, Ultrafast coupling of coherent phonons with a nonequilibrium electron-hole plasma in GaAs, Phys. Rev. B 91 (2015) 125201.

[43] R. Huber, F. Tauser, A. Brodschelm, M. Bichler, G. Abstreiter, A. Leitenstorfer, How many-particle interactions develop after ultrafast excitation of an electron-hole plasma, Nature 414 (2001) 286-9.

[44] X. Cui, C. Wang, A. Argondizzo, S. Garret-Roe, B. Gumhalter, H. Petek, Transient excitons at metal surfaces, Nat. Phys. 10 (2014) 505.

[45] V. M. Silkin, P. Lazić, N. Došlić, H. Petek, B. Gumhalter, Ultrafast electronic response of Ag(111) and Cu(111) surfaces: From early excitonic transients to saturated image potential, Phys. Rev. B 92 (2015) 155405, doi:10.1103/PhysRevB.92.155405, URL http://link.aps.org/doi/10.1103/PhysRevB.92.155405.

[46] A. Cavalleri, T. Dekorsy, H. H. W. Chong, J. C. Kieffer, R. W. Schoenlein, Evidence for a structurally-driven insulator-to-metal transition in $\mathrm{VO}_{2}$ : A view from the ultrafast timescale, Phys. Rev. B 70 (2004) 161102, doi:10.1103/PhysRevB.70.161102, URL http://link.aps.org/doi/10.1103/PhysRevB.70.161102.

[47] X. Yuan, W. Zhang, P. Zhang, Hole-lattice coupling and photoinduced insulator-metal transition in $\mathrm{VO}_{2}$, Phys. Rev. B 88 (2013) 035119, doi:10.1103/PhysRevB.88.035119, URL http://link.aps.org/doi/10.1103/PhysRevB.88.035119.

[48] A. Pashkin, C. Kübler, H. Ehrke, R. Lopez, A. Halabica, R. F. Haglund, R. Huber, A. Leitenstorfer, Ultrafast insulator-metal phase transition in $\mathrm{VO}_{2}$ studied by multiterahertz spectroscopy, Phys. Rev. B 83 (2011) 195120, doi:10.1103/PhysRevB.83.195120, URL http://link.aps.org/doi/10.1103/PhysRevB.83.195120.

[49] A. Leitenstorfer, Private communications, 2014/15.

[50] D. N. Basov, R. D. Averitt, D. van der Marel, M. Dressel, K. Haule, Electrodynamics of correlated electron materials, Rev. Mod. Phys. 83 (2011) 471 .

[51] P. W. Anderson, The Size of Localized States Near the Mobility Edge, Proc. Natl. Acad. Sci. 69 (1972) 1097.

[52] S. K. Sundaram, E. Mazur, Inducing and probing non-thermal transitions in semiconductors using femtosecond laser pulses, Nat. Mater. 1 (2002) 217-224.

[53] E. Hendry, M. Koeberg, M. Bonn, Exciton and electron-hole plasma formation dynamics in ZnO, Phys. Rev. B 76 (2007) 045214.

[54] J.-C. Deinert, D. Wegkamp, M. Meyer, C. Richter, M. Wolf, J. Stähler, Ultrafast Exciton Formation at the ZnO(1010) Surface, Phys. Rev. Lett. 113 (2014) 057602.

[55] P. Gaal, W. Kuehn, K. Reimann, M. Woerner, T. Elsaesser, R. Hey, Internal motions of a quasiparticle governing its ultrafast nonlinear response, Nature 450 (2007) 1210.

[56] H. Sezen, H. Shang, F. Bebensee, C. Yang, M. Buchholz, A. Nefedov, S. Heissler, C. Carbogno, M. Scheffler, P. Rinke, C. Wöll, Evidence for photogenerated intermediate hole polarons in $\mathrm{ZnO}$, Nat. Commun. 6 (2015) 6901.

[57] P. Baum, D.-S. Yang, A. H. Zewail, 4D Visualization of Transitional Structures in Phase Transformations by Electron Diffraction, Science 318 (5851) (2007) 788-792, doi:10.1126/science.1147724, URL http://www . sciencemag. org/content/318/5851/788. abstract.

[58] A. Cavalleri, C. Tóth, C. W. Siders, J. A. Squier, F. Ráksi, P. Forget, J. C. Kieffer, Femtosecond Structural Dynamics in VO 2 during an Ultrafast Solid-Solid Phase Transition, Phys. Rev. Lett. 87 (2001) 237401, doi:10.1103/PhysRevLett.87.237401, URL http://link.aps.org/doi/10.1103/PhysRevLett.87.237401.

[59] M. Hada, K. Okimura, J. Matsuo, Characterization of structural dynamics of $\mathrm{VO}_{2}$ thin film on c-Al-2O $\mathrm{O}_{3}$ using in-air time-resolved $x$-ray diffraction, Phys. Rev. B 82 (2010) 153401, doi:10.1103/PhysRevB.82.153401, URL http://link.aps.org/doi/10.1103/PhysRevB.82.153401.

[60] M. Hada, K. Okimura, J. Matsuo, Photo-induced lattice softening of excited-state $\mathrm{VO}_{2}$, Appl. Phys. Lett. 99 (5) 051903, doi: http://dx.doi.org/10.1063/1.3621900, URL http://scitation.aip.org/content/aip/journal/apl/99/5/10.1063/1.3621900.

[61] B. O'Callahan, A. C. Jones, J. H. Park, D. H. Cobden, J. M. Atkin, M. B. Raschke, Inhomogeneity of the ultrafast insulator-to-metal transition dynamics of $\mathrm{VO}_{2}$, Nat. Commun. 6 (2015) 6849.

[62] H. Dachraoui, N. Müller, G. Obermeier, C. Oberer, S. Horn, U. Heinzmann, Interplay between electronic correlations and coherent structural dynamics during the monoclinic insulator-to-rutile metal phase transition in $\mathrm{VO}_{2}$, J. Phys.: Condens. Matter 23 (43) (2011) 435402, URL http: //stacks. iop.org/0953-8984/23/i=43/a=435402.

[63] R. Yoshida, T. Yamamoto, Y. Ishida, H. Nagao, T. Otsuka, K. Saeki, Y. Muraoka, R. Eguchi, K. Ishizaka, T. Kiss, S. Watanabe, T. Kanai, J. Itatani, S. Shin, Ultrafast photoinduced transition of an insulating $\mathrm{VO}_{2}$ thin film into a nonrutile metallic state, Phys. Rev. B 89 (2014) 205114, doi:10.1103/PhysRevB.89.205114, URL http://link.aps.org/doi/10.1103/PhysRevB.89.205114.

[64] H. J. Zeiger, J. Vidal, T. K. Cheng, E. P. Ippen, G. Dresselhaus, M. S. Dresselhaus, Theory for displacive excitation of coherent phonons, Phys. Rev. B 45 (1992) 768-778, doi:10.1103/PhysRevB.45.768, URL http://link .aps .org/doi/10.1103/PhysRevB.45.768.

[65] T. K. Cheng, L. H. Acioli, J. Vidal, H. J. Zeiger, G. Dresselhaus, Modulation of a semiconductor to semimetal transition at 7 THz via coherent lattice vibrations., Appl. Phys. Lett. 62 (1993) 1901.

[66] M. Hase, M. Kitajima, S. Nakashima, K. Mizoguchi, Dynamics of Coherent Anharmonic Phonons in Bismuth Using High Density Photoexcitation., Phys. Rev. Lett. 88 (2002) 067401.

[67] E. e. Möhr-Vorobeva, Nonthermal Melting of a Charge Density Wave in TiSe 2 , Phys. Rev. Lett. 107 (2011) 036403. 
[68] K. Ishioka, M. Hase, M. Kitajima, L. Wirtz, A. Rubio, H. Petek, Ultrafast electron-phonon decoupling in graphite., Phys. Rev. B 77 (2008) 121402.

[69] D. M. e. Fritz, Ultrafast Bond Softening in Bismuth: Mapping a Solid's Interatomic Potential with X-rays., Science 315 (2007) $633-636$.

[70] E. S. Zijlstra, L. L. Tatarinova, M. E. Garcia, Laser-induced phonon-phonon interactions in bismuth., Phys. Rev. B 74 (2006) 220301.

[71] B. Mayer, C. Schmidt, A. Grupp, J. Bühler, J. Oelmann, R. E. Marvel, R. F. Haglund Jr., T. Oka, D. Brida, A. Leitenstorfer, P. A., Tunneling breakdown of a strongly correlated insulating state in $\mathrm{VO}_{2}$ induced by intense multiterahertz excitation, Phys. Rev. B 91 (2015) 235113.

[72] E. Riedel, C. Janiak, Anorganische Chemie, de Gruyter, 2007.

[73] J. Y. Suh, R. Lopez, L. C. Feldman, R. F. Haglund, Semiconductor to metal phase transition in the nucleation and growth of $\mathrm{VO}_{2}$ nanoparticles and thin films, J. Appl. Phys. 96 (2) (2004) 1209-1213, doi:http://dx.doi.org/10.1063/1.1762995, URL http://scitation.aip.org/content/aip/journal/jap/96/2/10.1063/1.1762995.

[74] J. Nag, R. F. Haglund Jr, Synthesis of vanadium dioxide thin films and nanoparticles, J. Phys.: Condens. Matter 20 (26) (2008) 264016, URL http: //stacks . iop.org/0953-8984/20/i=26/a=264016.

[75] J. Nag, E. A. Payzant, K. L. More, R. F. Haglund, Enhanced performance of room-temperature-grown epitaxial thin films of vanadium dioxide, Appl. Phys. Lett. 98 (25) 251916, doi:http://dx.doi.org/10.1063/1.3600333, URL http://scitation.aip.org/content/aip/journal/apl/98/25/10.1063/1.3600333.

[76] V. Klimov, I. Timofeeva, S. Khanin, E. Shadrin, A. Ilinskii, F. Silva-Andrade, Hysteresis loop construction for the metalsemiconductor phase transition in vanadium dioxide films, Tech. Phys. 47 (9) (2002) 1134-1139, doi:10.1134/1.1508078, URL http://dx.doi.org/10.1134/1.1508078.

[77] R. Lopez, T. E. Haynes, L. A. Boatner, L. C. Feldman, R. F. Haglund, Size effects in the structural phase transition of $\mathrm{VO}_{2}$ nanoparticles, Phys. Rev. B 65 (2002) 224113, doi:10.1103/PhysRevB.65.224113, URL http://link.aps.org/doi/10.1103/PhysRevB.65.224113.

[78] W. Fan, J. Cao, J. Seidel, Y. Gu, J. W. Yim, C. Barrett, K. M. Yu, J. Ji, R. Ramesh, L. Q. Chen, J. Wu, Large kinetic asymmetry in the metal-insulator transition nucleated at localized and extended defects, Phys. Rev. B 83 (2011) 235102, doi:10.1103/PhysRevB.83.235102, URL http://link.aps.org/doi/10.1103/PhysRevB.83.235102.

[79] G. Leahu, R. Li Voti, C. Sibilia, M. Bertolotti, Anomalous optical switching and thermal hysteresis during semiconductor-metal phase transition of $\mathrm{VO}_{2}$ films on Si substrate, Appl. Phys. Lett. 103 (23) 231114, doi:http://dx.doi.org/10.1063/1.4838395, URL http://scitation.aip.org/content/aip/journal/apl/103/23/10.1063/1.4838395.

[80] D. Wegkamp, D. Brida, S. Bonora, G. Cerullo, J. Stähler, M. Wolf, S. Wall, Phase retrieval and compression of low-power white-light pulses, Appl. Phys. Lett. 99 (10) 101101, doi:http://dx.doi.org/10.1063/1.3635396, URL http://scitation.aip.org/content/aip/journal/apl/99/10/10.1063/1.3635396.

[81] J. Faure, J. Mauchain, E. Papalazarou, W. Yan, J. Pinon, M. Marsi, L. Perfetti, Full characterization and optimization of a femtosecond ultraviolet laser source for time and angle-resolved photoemission on solid surfaces, Rev. Sci. Instrum. 83 (4) 043109 , doi: http://dx.doi.org/10.1063/1.3700190, URL http://scitation.aip.org/content/aip/journal/rsi/83/4/10.1063/1.3700190.

[82] D. Wegkamp, Ultrafast electron dynamics and the role of screening, Ph.D. thesis, Freie Universität Berlin, 2015.

[83] S. Wall, D. Wegkamp, L. Foglia, K. Appavoo, J. Nag, R. Haglund, J. Stähler, M. Wolf, Ultrafast changes in lattice symmetry probed by coherent phonons, Nat. Commun. 3 (2012) 721, URL http://dx.doi.org/10.1038/ncomms 1719

[84] W. P. Hsieh, M. Trigo, D. A. Reis, G. Artioli, L. Malavasi, W. L. Mao, Evidence for photo-induced monoclinic metallic VO2 under high pressure, Appl. Phys. Lett. 104 (2014) 021917.

[85] E. Knoesel, T. Hertel, M. Wolf, G. Ertl, Femtosecond dynamics of electronic excitations of adsorbates studied by two-photon photoemission pulse correlation: $\mathrm{CO} / \mathrm{Cu}(111)$, Chem. Phys. Lett. 240 (1995) 409 - 416, ISSN 0009-2614, doi:http://dx.doi.org/10.1016/00092614(95)00527-B, URL http://www.sciencedirect.com/science/article/pii/000926149500527B.

[86] C. Schmuttenmaer, C. C. Miller, J. Herman, J. Cao, D. Mantell, Y. Gao, R. Miller, Femtosecond time-resolved photoemission study of hot electron relaxation at the GaAs(100) surface, Chem. Phys. 205 (1996) 91 - 108, ISSN 0301-0104, doi:http://dx.doi.org/10.1016/03010104(95)00328-2, URL http://www.sciencedirect.com/science/article/pii/0301010495003282, surface Reaction Dynamics.

[87] T. Hertel, E. Knoesel, M. Wolf, G. Ertl, Ultrafast Electron Dynamics at Cu(111): Response of an Electron Gas to Optical Excitation, Phys. Rev. Lett. 76 (1996) 535-538, doi:10.1103/PhysRevLett.76.535, URL http://link. aps . org/doi/10.1103/PhysRevLett.76.535.

[88] R. Ernstorfer, Spectroscopic investigation of photoinduced heterogeneous electron transfer, Ph.D. thesis, Freie Universität Berlin, 2004.

[89] A. Oschlies, R. W. Godby, R. J. Needs, GW self-energy calculations of carrier-induced band-gap narrowing in n-type silicon, Phys. Rev. B 51 (1995) 1527-1535.

[90] R. Abram, G. Rees, B. Wilson, Heavily doped semiconductors and devices, Adv. Phys. 27 (1978) 799-892.

[91] J. Wagner, Band-gap narrowing in heavily doped silicon at 20 and $300 \mathrm{~K}$ studied by photoluminescence, Phys. Rev. B 32 (1985) $1323-1325$.

[92] Y. Dou, T. Fishlock, R. G. Egdell, D. S. L. Law, G. Beamson, Band-gap shrinkage in n-type-doped CdO probed by photoemission spectroscopy, Phys. Rev. B 55 (1997) R13381-R13384.

[93] C. D. Spataru, L. X. Benedict, S. G. Louie, Ab initio calculation of band-gap renormalization in highly excited GaAs, Phys. Rev. B 69 (2004) 205204.

[94] M. Rini, Z. Hao, R. W. Schoenlein, C. Giannetti, F. Parmigiani, S. Fourmaux, J. C. Kieffer, A. Fujimori, M. Onoda, S. Wall, A. Cavalleri, Optical switching in $\mathrm{VO}_{2}$ films by below-gap excitation, Appl. Phys. Lett. 92 (18) 181904, doi:http://dx.doi.org/10.1063/1.2921784, URL http://scitation.aip.org/content/aip/journal/apl/92/18/10.1063/1.2921784.

[95] L. Xian, Private communications, 2015.

[96] H. W. Verleur, A. S. Barker, C. N. Berglund, Optical Properties of $\mathrm{VO}_{2}$ between 0.25 and 5 eV, Phys. Rev. 172 (1968) 788-798, doi: 10.1103/PhysRev.172.788, URL http://link.aps.org/doi/10.1103/PhysRev.172.788.

[97] J. C. Parker, Raman scattering from $\mathrm{VO}_{2}$ single crystals: A study of the effects of surface oxidation, Phys. Rev. B 42 (1990) $3164-3166$, doi:10.1103/PhysRevB.42.3164, URL http://link.aps.org/doi/10.1103/PhysRevB.42.3164.

[98] J. M. Atkin, S. Berweger, E. K. Chavez, M. B. Raschke, J. Cao, W. Fan, J. Wu, Strain and temperature dependence of the in- 
sulating phases of $\mathrm{VO}_{2}$ near the metal-insulator transition, Phys. Rev. B 85 (2012) 020101, doi:10.1103/PhysRevB.85.020101, URL http://link.aps.org/doi/10.1103/PhysRevB.85.020101.

[99] S. A. Kovalenko, A. L. Dobryakov, J. Ruthmann, N. P. Ernsting, Femtosecond spectroscopy of condensed phases with chirped supercontinuum probing, Phys. Rev. A 59 (1999) 2369-2384, doi:10.1103/PhysRevA.59.2369, URL http://link.aps.org/doi/10.1103/PhysRevA.59.2369.

[100] M. Hase, K. Ishioka, J. Demsar, K. Ushida, M. Kitajima, Ultrafast dynamics of coherent optical phonons and nonequilibrium electrons in transition metals, Phys. Rev. B 71 (2005) 184301. 